\title{
SYMBIOTIC SENSING
}

Exploring and Exploiting Cooperative Sensing in Heterogeneous Sensor Networks

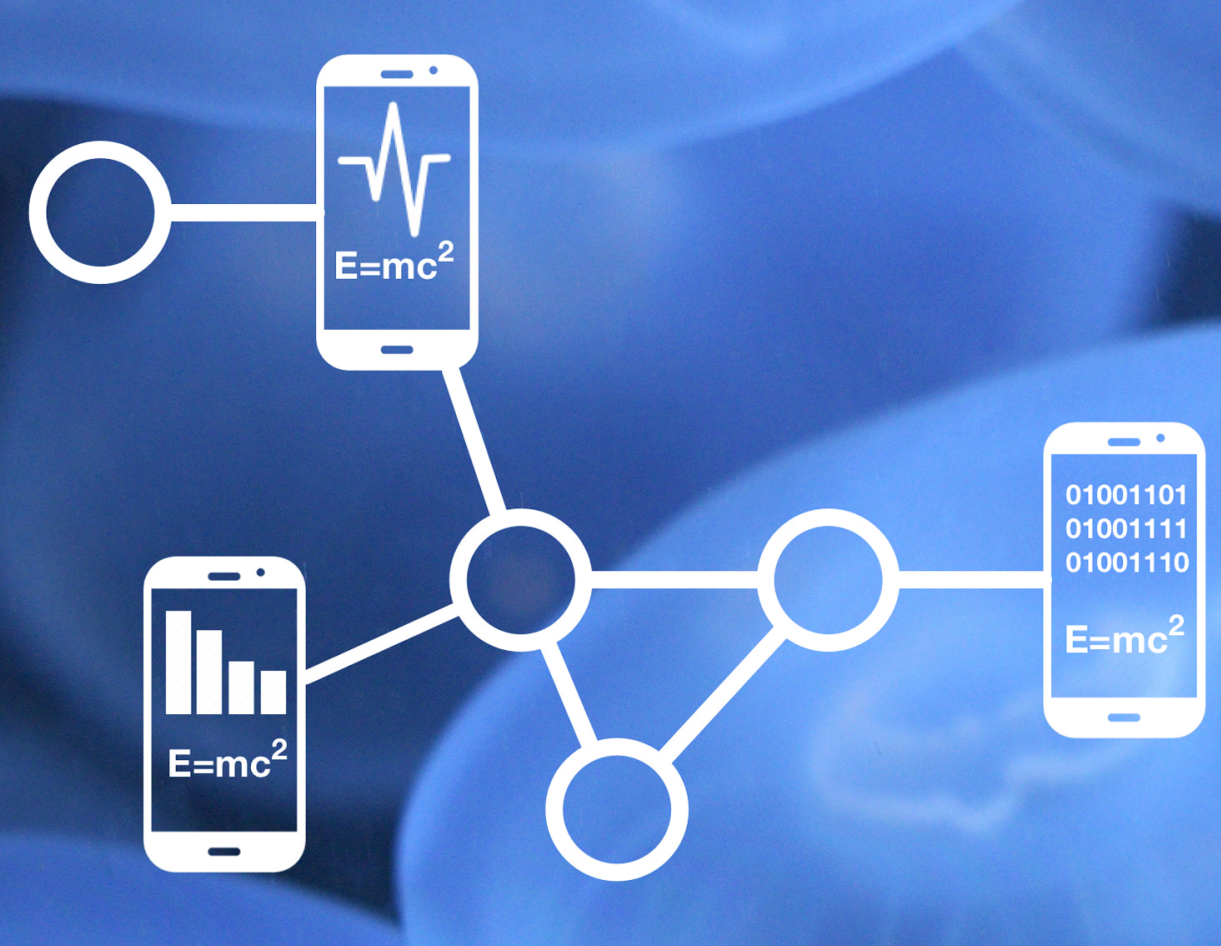

\section{LÊ VIẾT ĐỨC}





\section{SYMBIOTIC SENSING}

\section{Exploring and Exploiting Cooperative Sensing}

in Heterogeneous Sensor Networks

Lê Viết Đức 
Graduation committee:

Chairman:

Supervisor:

Referee:

Members:

Prof. dr. ir. B.R.H.M. Haverkort

Prof. dr. ir. M.R. van Steen

Prof. dr. M. Kumar

Prof. dr. K. Langendoen
Prof. dr. Peter M.G. Apers

Prof. dr. ing. Paul J.M. Havinga

Ir. Hans Scholten

University of Twente

University of Twente

Rochester Institute of Technology

Delft University of Technology

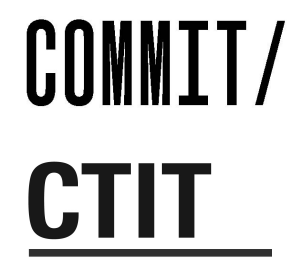

This research is supported by the SenSafety project within the Dutch National Program COMMIT.

CTIT Ph.D. Thesis Series No. 16-403

Centre for Telematics and Information Technology University of Twente

P.O. Box 217, 7500 AE, Enschede, The Netherlands

ISSN 1381-3617

ISBN 978-90-365-4185-5

DOI: $10.3990 / 1.9789036541855$

Abstract translation: Hans Scholten

Cover design: Lê Viết Đức

Printed by CPI - Koninklijke Wöhrmann

Copyright (C) Lê Viết Đức

All rights reserved. No part of this book may be reproduced or transmitted, in any form or by any means, electronic or mechanical, including photocopying, microfilming, and recording, or by any information storage or retrieval system, without the prior written permission of the author. 


\title{
EXPLORING AND EXPLOITING COOPERATIVE SENSING IN HETEROGENEOUS SENSOR NETWORKS
}

\author{
DISSERTATION
}

to obtain

the degree of doctor at the University of Twente, on the authority of the rector magnificus,

Prof. dr. H. Brinksma, on account of the decision of the graduation committee, to be publicly defended on Thursday 01 September 2016 at 14.45

by

\section{Lê Viết Đức}

born on 21 March 1979

in Tuyen Hoa, Quang Binh, Vietnam 
This dissertation is approved by:

Prof. dr. ing. Paul J.M. Havinga (supervisor)

Ir. Hans Scholten (referee) 


\section{Acknowledgments}

This dissertation would not have been possible without the help of many kind, talented, and hardworking people. I would first like to express my sincere gratitude to my supervisors, Paul Havinga and Hans Scholten for continuous support of my Ph.D study and related research, for their immense knowledge and guidance.

Besides my supervisors, many thanks to my committee members: Boudewijn Haverkort, Maarten van Steen, Mohan Kumar, and Koen Langendoen, for their insightful comments, but also the hard questions which incented me to widen my research from various perspectives.

My sincere thanks also go to Nirvana Meratnia, who provided me an opportunity to enhance my research. Without her precious support it would not be possible to complete this dissertation.

I am also grateful to work with many outstanding colleagues and collaborators. Specifically, I would like to thank Jacob Kamminga, Kallol Das, Wouter van Kleunen, Helena Bisby, Nguyen Duc Thang, Hung Ngo, Vien Ngo, Thuong Nguyen, David Nguyen, and Dinh Phung, for their ever present help and feedback. It has been great to have the stimulating discussions during lunches, tea breaks, group seminars, and events. Thanks to all of them for their assistance and insight: Okan Turkes, Fatjon Seraj, Jan-Pieter Meijers, Muhammad Shoaib, Eyuel Debebe Ayele, Alexander Belov, Arta Dilo, Ramon Schwartz, Niels Moseley, Berend Jan Van Der Zwaag, Pouria Zand, and Majid Bahrepour.

I would also like to thank our wonderful secretaries and HR staffs, Nicole Baveld, Thelma Nordholt, Marlous Weghorst, Odette Scholten, and Ellen van Erven for their excellent administrative support.

Last but not least, I would like to thank my family for their patience, support, and encouragement. My wife and my son have provided unprecedented understanding, support, and endless happiness over the years. I am also very grateful for the encouragement and love from my parents and brothers.

Lê Viết Đức

Enschede, August 2016 



\section{Abstract}

During the last several years we have witnessed the emergence of smartphonebased sensing applications that include activity recognition, urban sensing, social sensing, and health monitoring. In fact, most smartphones have various sensors, wireless communication interfaces, a large memory capacity, powerful processors and operating systems. These features of smartphones have intrigued researchers to develop sensing systems in which smartphones support or even replace the traditional devices in Wireless Sensor Networks (WSNs). However, smartphones are not deliberately designed as dedicated sensing devices. Using smartphones as sensing devices opens new sensing possibilities, but also comes with new challenges, which are introduced below.

When numerous applications are running on a smartphone simultaneously, they are likely to conflict when acquiring resources such as sensors, memories, battery, and bandwidth. For example, sensing applications might fail when simultaneously using sensors on Android smartphones since the sensors like microphones are exclusive, and cannot be accessed by multiple applications at the same time. Moreover, continuously sampling data would result in the batteries of smartphones being depleted quickly.

In addition, smartphones are non-deterministic platforms by design, which usually add considerable uncertainties to their sensory measurements. For example, acoustic data measured by the microphones of most Android smartphones are subject to considerable delay and clock synchronization errors of up to hundreds of miliseconds. Such tolerances lead to inaccurate estimates of distances between sound sources and the microphones, which are measured based on time of arrival.

Furthermore, the dynamic mobility of smartphones carried by the users introduces daunting challenges to information retrieval. These challenges include the dramatic change of the sensing contexts, the sensing locations, and the background noises. For example, audio data of a person laughing in a pub is different from that recorded on a street. These unexpected noises significantly influence the development of human-centric sensing systems. 
Short-range radios such as WiFi and Bluetooth offer direct communication of sending data among phones by a method such as the store-carry-forward paradigm. However, this approach is challenging because of the dynamic mobility of smartphones carried by users, of which movement patterns are hard to be predicted.

To this end, we designed a symbiotic-like architecture of smartphone sensing systems using a cooperative and distributed approach through three main stacks: data sampling, data processing and data dissemination. The architecture imitates the symbiosis in nature to pave the way for multiple sensing applications to run seamlessly on a smartphone. We explore and exploit opportunities given by smartphones to overcome the above challenges. We address techniques to support building and using smartphone-based sensing systems in the context of Heterogenous Sensor Networks (HSNs). We consider the increasing number of smartphones, the growing number of applications, the diversity of sensing capabilities of smartphones, and the dynamic mobility of the smartphones' users. The results of simulations and experiments of our proposed techniques are consistent with the theoretical analysis. For further research, we will elaborate and integrate these algorithms into a complete sensing system for smartphone-based sensing applications in smart cities. 


\section{Samenvatting}

De laatste jaren zijn we getuige geweest van de opkomst van applicaties voor smartphones, waaronder activiteitenherkenning, monitoring van iemand's gezondheid, "urban sensing" en "social sensing" (waarnemingen in een stedelijke setting van, onder andere, menselijk gedrag). Dit wordt mogelijk gemaakt omdat de meeste moderne mobiele telefoons een veelheid aan sensoren bezitten, draadloos kunnen communiceren, voorzien zijn van een groot geheugen en een krachtig besturingssysteem hebben. Deze bijzondere kenmerken hebben onderzoekers geïnspireerd tot het maken van toepassingen normaal voorbehouden aan traditionele draadloze sensornetwerken. Hoewel smartphones onvoorziene mogelijkheden hebben voor deze nieuwe toepassingen zijn ze er niet specifiek voor ontworpen en bestaan er grote nieuwe uitdagingen bij het ontwikkelen van deze toepassingen.

Als meerdere applicaties tegelijk actief zijn op een smartphone is het waarschijnlijk dat er conflicten ontstaan over het gebruikt van sensoren, geheugen, energie en bandbreedte. Een voorbeeld is het gebruik van de microfoon door meerdere applicaties tegelijk. In de huidige systemen is dat onmogelijk omdat de microfoon slechts exclusief door een applicatie gebruikt kan worden. Bovendien zal een continu gebruik van de microfoon al snel leiden tot een lege batterij.

Smartphones zijn in essentie non-deterministisch, waardoor metingen uitgevoerd door sensoren in de telefoon een grote mate van onzekerheid vertonen. Bijvoorbeeld, door het optreden van een vertraging van onbepaalde lengte door het gebruik van "sampling"-buffers en fouten in de kloksynchronisatie kunnen er fouten van enkele honderden milliseconden optreden bij het meten van geluid door Android smartphones. Het meten van de afstand tot een geluidsbron is hierdoor niet betrouwbaar.

Doordat eigenaars van smartphones, en dus ook de smartphones, mobiel zijn en zich niet voortdurend op dezelfde plek ophouden, verandert de context waarin metingen worden verricht voortdurend. Omdat achtergrondgeluid en akoestiek anders zijn, is bijvoorbeeld het gemeten geluid van een lachend per- 
soon in een café anders dan dat van dezelfde persoon op straat. Dit fenomeen heeft aanmerkelijke invloed op het ontwerpen van sensorsystemen waar de mens centraal staat.

Radio's voor korte afstanden zoals WiFi en Bluetooth zijn in principe geschikt voor directe communicatie tussen smartphones onderling, waarbij gebruik gemaakt zou kunnen worden van een "store-carry-forward" methode. Bij deze methode wordt (ontvangen) informatie door een smartphone meegenomen totdat deze weer op een andere locatie kan worden doorgegeven aan de volgende smartphone. De onvoorspelbare mobiliteit en bewegingspatronen van de eigenaars vormt echter een grote uitdaging.

Om deze uitdagingen aan te gaan is een nieuwe architectuur voor smartphones ontworpen die gebaseerd is op symbiotische principes, waarbij samenwerking en distributie van taken centraal staan. Hierdoor wordt het mogelijk dat meerdere programma?s op een of meerdere smartphones beperkte bronnen kunnen delen en tegelijk actief kunnen zijn op een manier die energieefficiënt is. De architectuur bestaat uit drie onderdelen voor data "sampling", data "processing" en data disseminatie. Dit proefschrift behandelt technieken die het bouwen en het gebruik van smartphone-sensorsystemen ondersteunen. Daarbij wordt uitgegaan van toenemende aantallen applicaties en steeds krachtiger smartphones, een grote diversiteit aan sensoren in smartphones, en mobiliteit van de eigenaars van smartphones. Resultaten van simulaties en experimenten zijn consistent met de theoretische analyses. Het proefschrift gaat in op toekomstig onderzoek, waar de ontwikkelde algoritmes worden geïntegreerd in complete sensorapplicaties voor intelligente steden ("smart cities"). 


\section{Contents}

1 Introduction $\mathbf{1}$

1.1 Heterogeneous Smartphone Sensor Networks . . . . . . . . . . . . 2

1.2 Opportunities of Smartphone-Based Platforms . . . . . . . . . . 4

1.2.1 Services and Concurrent Programming . . . . . . . . . 5

1.2.2 Proliferation of Smartphones . . . . . . . . . . . . . 5

1.2.3 Human-like Mobility Patterns . . . . . . . . . . . . . . 6

1.3 Research Objectives . . . . . . . . . . . . . . . . 6

1.4 Contributions . . . . . . . . . . . . . . . . . . 11

1.5 Dissertation Organisation . . . . . . . . . . . . . . . 15

2 State of the Art 17

2.1 Introduction . . . . . . . . . . . . . . . . . . 17

2.2 Sensing Categories . . . . . . . . . . . . . . . . . . . . . . . 18

2.2.1 Sensing Paradigms . . . . . . . . . . . . . . . . . . . 19

2.2.2 Smartphone-based Computing Approaches . . . . . . . . . . 20

2.2.3 Smartphone Sensor Network Communication . . . . . . . . . 21

2.3 Smartphone-based Sensing Applications . . . . . . . . . . . . . . . . . 21

2.3.1 Personal Sensing . . . . . . . . . . . . . . . . . . . . 24

2.3.2 Social Behavior Sensing . . . . . . . . . . . . . . . . . . . 24

2.3.3 Environmental Sensing . . . . . . . . . . . . . . . . . . 24

2.3.4 Infrastructure Sensing . . . . . . . . . . . . . . . 24

2.4 Open Research Areas . . . . . . . . . . . . . . . . . . . . . . . 24

2.4 .1 Hybrid Sensing Paradigm . . . . . . . . . . . . . . . . . 25

2.4 .2 Cooperative Sensing Systems . . . . . . . . . . . . . . . . . 25

2.4 .3 Online Learning Algorithms . . . . . . . . . . . . . . . . . . 26

2.4 .4 Timely Data Processing . . . . . . . . . . . . . . . 26

2.4 .5 Data Gathering . . . . . . . . . . . . . . . . . 26

2.4 .6 Energy Efficiency . . . . . . . . . . . . . . . . . . . . . 27

2.4 .7 Privacy Protection . . . . . . . . . . . . . . . . . . . . 27

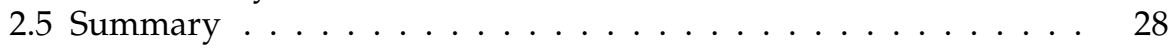


3 Cooperative Hybrid Sensing 29

3.1 Introduction . . . . . . . . . . . . . . . . . . . . . . . . 29

3.2 Symbiotic Sensing . . . . . . . . . . . . . . . 30

3.2 .1 Sensing Paradigms . . . . . . . . . . . . . . 31

3.2 Motivation . . . . . . . . . . . . . . . 32

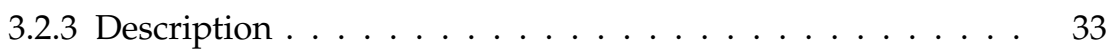

3.2 .4 Problem Formulation . . . . . . . . . . . . . . . . . . . . 35

3.2 .5 Evaluation Model . . . . . . . . . . . . . . . . . . . . . . . 37

3.2.6 Quantitative Evaluation . . . . . . . . . . . . . 41

3.3 Distributed Cooperative Sensing Architecture . . . . . . . . . . . . 47

3.4 Summary ........................... 49

4 Cooperative Data Sampling $\quad 51$

4.1 Introduction . . . . . . . . . . . . . . . . 51

4.2 Related Work . . . . . . . . . . . . . . . . . . . . . . . . 53

4.3 Sampling Architecture . . . . . . . . . . . . . . . . . . . 54

4.3.1 Cross-Application Sensor Service . . . . . . . . . . . . . 54

4.3.2 Smartphone-Context Detector . . . . . . . . . . . . . . 56

4.3 .3 Event States Detector . . . . . . . . . . . . . . . . . . . . . 56

4.3 .4 User Interaction . . . . . . . . . . . . . . . . . . . . . . 57

4.3.5 Sampling Management . . . . . . . . . . . . . . . . . 57

4.4 Minimum Active Duration Sensing Scheduling . . . . . . . . . . . . 58

4.4.1 Change Points of Context States . . . . . . . . . . . . . 58

4.4.2 Minimum Active Duration Sensing Scheduling (MASS) . . . 59

4.4 .3 Mathematical Formulation . . . . . . . . . . . . . . . . 60

4.5 Context Change Detection . . . . . . . . . . . . . . . 63

4.5.1 Histogram Likelihood Change Detection . . . . . . . . . . 63

4.5 .2 Complexity . . . . . . . . . . . . . . 65

4.5 .3 Evaluation Metrics . . . . . . . . . . . . . . . . . . 66

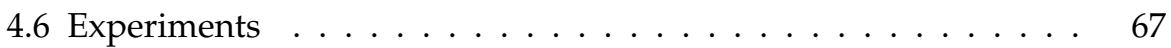

4.6 .1 Experimental Setup . . . . . . . . . . . . . . . . . . 67

4.6 .2 Experiment Results . . . . . . . . . . . . . . . . . . . . 68

4.7 Summary . . . . . . . . . . . . . . . . 75

5 Non-deterministic Data Processing $\quad 77$

5.1 Introduction . . . . . . . . . . . . . . . . . . 78

5.2 Non-determinism in Smartphone-based Sensing . . . . . . . . . . . 79

5.2 .1 Time synchronization . . . . . . . . . . . . . . . . . . 80

5.2 .2 Sensing latency . . . . . . . . . . . . . . 80 
5.2.3 Experimental Evaluation . . . . . . . . . . . . . . . . 81

5.3 Non-deterministic Algorithms for Smartphone-based Sensing . . . 84

5.3.1 Sound Source Localization Problem . . . . . . . . . . . . . 84

5.3.2 Time-of-Different Arrival Localization . . . . . . . . . . . . . 85

5.3.3 Non-deterministic Localization Approach . . . . . . . . . . 87

5.4 Testbed Experiment . . . . . . . . . . . . . . . . . . . . . . 90

5.4.1 Experimental Setup . . . . . . . . . . . . . . . . . . . 90

5.4 .2 Experimental Results . . . . . . . . . . . . . . . . . 91

5.5 Summary . . . . . . . . . . . . . . . . . . . 94

6 Robust Data Dissemination 97

6.1 Introduction . . . . . . . . . . . . . . . . . . . . . . . . . 98

6.2 Opportunistic Routing Approaches . . . . . . . . . . . . . . . . 99

6.2.1 Routing Without Infrastructure Assistance . . . . . . . . . . . 99

6.2.2 Routing With Infrastructure Assistance . . . . . . . . . . . 101

6.2.3 Routing for Heterogenous Sensor Networks . . . . . . . . . 101

6.2 .4 Evaluation Metrics . . . . . . . . . . . . . . . . . . . 102

6.3 Mobility Models . . . . . . . . . . . . . . . . . . . . . . . 103

6.3.1 Human-like Mobility . . . . . . . . . . . . . . . . . . . . 103

6.3 .2 Model Evaluation . . . . . . . . . . . . . . . . . . . . . 104

6.4 Unified Routing for Heterogeneous Networks . . . . . . . . . . 105

6.4 .1 Unified Parameters . . . . . . . . . . . . . . . . . . . . 107

6.4 .2 Unified Routing . . . . . . . . . . . . . . . . . . . . . 108

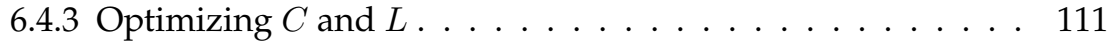

6.4.4 Simulation . . . . . . . . . . . . . . . . . . . . 113

6.5 Location-based Routing with Human Mobility Behaviours . . . . . 120

6.5 .1 Problem Formulation . . . . . . . . . . . . . . . . . . 121

6.5.2 Unsupervised Learning Approaches . . . . . . . . . . . 123

6.5 .3 Simulation . . . . . . . . . . . . . . . . . . . . . 127

6.6 Summary . . . . . . . . . . . . . . . . . . . . 132

7 Conclusions 133

7.1 Evaluation of Contributions . . . . . . . . . . . . . . 133

7.2 Conclusions . . . . . . . . . . . . . . . . . . . . . . . 135

7.3 Future Research Directions . . . . . . . . . . . . . . . . 138

$\begin{array}{ll}\text { Bibliography } & 141\end{array}$ 



\section{CHAPTER 1}

\section{Introduction}

The emerging wave of technology in human-centric devices such as smartphones has paved the way for a large number of sensing applications, such as activity recognition, environmental sensing, social sensing, and health monitoring. In particular, most people nowadays frequently carry at least one smartphone, which has various off-the-shelf sensors and wireless communications. Such pervasiveness of onboard sensors brought about new possibilities to replace costly and dedicated sensors in traditional wireless sensor networks. However, such proliferation also brings new challenges to smartphone-based sensing research. Firstly, many users are not willing to participate in sensing networks with their smartphones because of battery and privacy concerns. Although the battery capacity of modern smartphones has significantly increased, the smartphones need to be recharged more frequently because of heavier software, more hardware, more applications and more frequent usage. Therefore, data sampling should be energy efficient. We remark that privacy issues are themselves broad research questions to be addressed by the community and this dissertation will not address them in detail, albeit we recognize their importance. Secondly, smartphones are still not in situ designed for dedicated sensing in spite of the fact that they are getting more and more powerful. Being non-deterministic, smartphone-based platforms significantly affect the performance of time-critical applications. The sensing capabilities also vary among different smartphones. This sensing diversity can be caused by hardware specifications, manufacturers and operating systems, especially in the case of smartphones with low-quality components and devices. Thirdly, smartphones are regularly carried by the users, whose movement patterns are hard to be predicted accurately. The dynamic mobility poses another serious challenge to transferring and processing data for location-based applications.

This dissertation addresses the aforementioned barriers which challenge the application of smartphone sensing in the real world by exploring and ex- 
ploiting opportunities provided by smartphones and Heterogenous Sensor Networks (HSNs). In particular, we propose cooperative data sampling methods for smartphone-based platforms with regard to energy saving and computing. Then, we study cooperative data processing with smartphones through sound source localization using Android devices, which has not fully exploited by other works. Finally, we enhance message dissemination among smartphones to broadcast the sensing results in a HSN, which is constrained by intermittent end-to-end connectivity.

The remainder of this chapter is organized as follows. Section 1.1 describes smartphone-based sensing networks that we address. In Section 1.2 we discuss the opportunities offered by smartphones that have not been fully exploited. The main objectives are defined in Section 1.3. Section 1.4 shortly clarifies our main contributions in this dissertation. Finally, the outline of this dissertation is described in Section 1.5.

\subsection{Heterogeneous Smartphone Sensor Networks}

The fact that sensors are everywhere and are integrated into most devices has brought the conventional Wireless Sensor Network (WSN) into a new era, namely Heterogenous Sensor Network (HSN). Sensing tasks in conventional sensor networks are usually performed by a certain set of specific sensing devices, of which sensors are dedicatedly designed for determined measurements in a particular network type. Conversely, in the era of HSNs, most devices participate in sensing and performing tasks as long as they have an opportunity to do so. These devices can be smartphones, smartwatches, wearables, smart lampposts, etc. As the result, new sensing systems become more reliable, scalable and robust, while still achieving acceptable accuracy. The reason is that they do not totally rely on particular devices or infrastructures, especially when a disaster or catastrophe happens.

Among the new sensing devices, smartphones equipped with sensors and short-range wireless communication have quickly pervaded our world and bring new opportunities as well as new challenges to research in pervasive systems. Using smartphones in a sensing system can achieve a similar or even better result than conventional sensor modules since smartphones are being used numerously and pervasively. Consequently, hardware and deployment costs are significantly reduced. This advantage has intrigued researchers to utilize smartphones to develop new sensing systems.

Although technologies have been improved significantly, smartphones still 
have limits on sensing capability, especially for urban sensing applications such as disaster management, public security enforcement, pollution surveillance, road monitoring, and healthcare applications. Reliability, robustness, and accuracy are critical requirements in such applications. Failure to meet these requirements might lead to undesirable, unpredictable, and catastrophic consequences. Therefore, we consider smartphone-based sensing applications in the context of HSNs, of which a possible architecture is shown in Fig. 1.1.

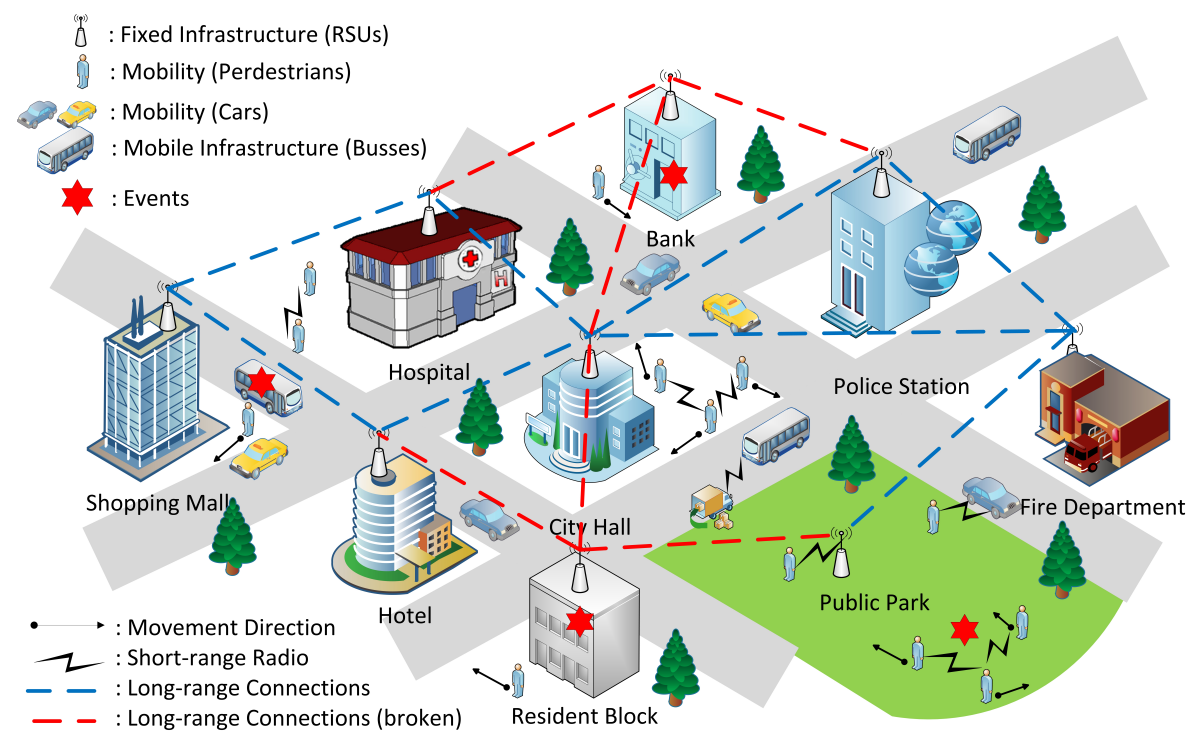

Figure 1.1: System Architecture of Smartphones and Heterogeneous Sensor Networks.

Since event processing is of fundamental importance to many smartphonebased sensing systems, we address architecture and techniques to support eventdriven applications. The system architecture consists of three main components: sensing devices, communication networks and a database centre. The sensing component mainly comprises of smartphones that are carried by users in a city. Besides smartphones, other types of sensor platforms also can be deployed to enhance the sensing capability of the system. The communication component is the wireless infrastructures that can communicate with avail- 
Introduction

able radio interfaces of smartphones. Although most smartphones in modern cities have mobile Internet subscriptions, we consider only short-range radios such as WiFi and Bluetooth which still work when infrastructures are damaged. Through the communication channels, the sensed information are disseminated among smartphones for distributed processing and gathered at a database centre for further processing and management.

Overall, HSNs detect an event mainly based on a crowd of smartphones. Assume that there is an event that happens at one of the locations marked by " $\star$ " and surrounded by a group of smartphones as well as fixed infrastructurebased sensors (see Fig. 1.1). First, smartphones cooperatively detect and collect relative information of the event considering energy efficiency. The data measured by each device are exchanged among smartphones in the group through short-range radio interfaces such as WiFi or Bluetooth. The measurements will be cooperatively processed by the smartphones in the group. After that, the results of the processing are sent to the data sink (base station) and other devices by using an opportunistic routing protocol to cope with intermittent connectivity. The message is transferred towards the destinations with the store-carryforward paradigm. As a result, the event information is gradually broadcasted to those interested in the event through the HSN that includes smartphones, vehicles and road-based units. A higher level of information retrieval may be executed at a database server.

\subsection{Opportunities of Smartphone-Based Platforms}

Sensing with smartphones, especially in continuous sensing, is a burden for smartphones and users in terms of resource consumption, time, communication costs, users' efforts, experience, and privacy. Previous work attempts to mitigate the burden by placing it on either the users under the opportunistic sensing schemes [1] or the smartphones under the participatory sensing schemes [2]. In addition, most smartphone-based sensing systems either locally log data on smartphones or directly send data to a central server for the backend processing. Later work attempt to process data in smartphone-based platforms; nevertheless, the data are processed separately without collaborating with neighboring smartphones. In fact, sampling, processing, and mining data can be done within a cluster of smartphones in a cooperative and distributed manner. Obviously, cooperative distributed schemes and smartphones have certain merits such as robustness and real-time performance. In this dissertation, we explore and exploit the following opportunities given by 
smartphones to improve the sensing performance.

\subsubsection{Services and Concurrent Programming}

Sensor services and concurrent programming have enabled sensing applications to run and access the sensor hardware simultaneously. Inspired by the symbiosis of natural living beings, we address reducing the burden of both the smartphones and users by exploiting the available opportunities offered by sharing sensing resources among applications. For example, environmental sound classification and user-mood recognition are preferably carried out whenever the user is making a phone call. By this mean, the emotion sensing piggybacks the phone call that includes environmental sounds and the user's voice to avoid jeopardizing smartphone's usage and battery. We name our proposed sensing paradigm symbiotic sensing. We build this new paradigm upon our hypothesis, "performing sensing tasks benefiting from given opportunities will mitigate the tradeoff between resource consumption and performance, and lower the burden placed on both users and smartphones". To the best of our knowledge, none of the previous work addresses this new paradigm. From our analysis with mathematical models given statistical data, we found that symbiotic sensing is suitable for sensing when smartphones are abundant.

\subsubsection{Proliferation of Smartphones}

We have witnessed significant development of smartphones during the last several years. In addition, users have spent more time with their smartphones. A report from Nielsen.com [3] reveals that the monthly time spent per person on smartphone applications has risen $63 \%$ in two years, from approximate 15 minutes per day in 2012 to approximate 25 minutes per day in 2014 . This proliferation provides an opportunity to collaborate applications for efficient sensing. Therefore, we address smartphone-based sensing systems through a cooperative distributed scheme. In particular, we develop techniques to allow performing online adaptive sampling and processing by collaborating sensors among smartphones. The results enable deploying smartphone-based sensing applications on a large scale more efficiently in terms of energy consumption and reliability. 


\subsubsection{Human-like Mobility Patterns}

As smartphones are often carried by the users, they have almost a similar movement pattern as that of the user. Using the merits given by the mobility, sensory data or sensing messages can be disseminated in a heterogeneous network through the store-carry-forward scheme using short-range radios. This approach provides a better reliability and robustness since it can still work if the infrastructure is destroyed by a nature disaster or sabotaged by criminals. In addition, since smartphone mobility is usually similar to that of its user, learning the mobility behavior of the users would enhance the sensing performance in terms of sensing strategy and data dissemination.

\subsection{Research Objectives}

The primary objective of this dissertation is to study solutions to improve the performance of smartphone-based sensing applications in HSNs in terms of accuracy, latency, reliability, robustness, and energy consumption. Although we recognize the importance of security and privacy issues, they are themselves broad research questions to be addressed by the community and this dissertation will not address them in detail. Instead, we aim to provide practical sensing techniques that work seamlessly in both normal and emergency conditions. We target sensing systems mainly based on smartphone platforms as we envision that smartphones will prevail over standard sensor nodes in HSNs. Such proliferation of smartphones also let us assume that there are often abundant smartphones sensing the same event simultaneously. We also assume that the firmware and physical implementation of integrated sensors and wireless interfaces on smartphones are off-the-shelf. That means we only need to deal with the data processing and in-network routing algorithms, which include the heterogeneity of sensors and devices.

With regard to the aforementioned scope, the main research question of this dissertation is:

\section{Research Question}

When ulitilizing smartphones as primary sensor nodes in heterogeneous sensor networks, what kinds of opportunities can be explored, and what techniques can be used to exploit such opportunities to improve the performance of the systems in terms of accuracy, latency, reliability, robustness, scalability, and energy consumption? 
With regard to the generic architecture of a sensing system, we address the aforementioned research question through three systematic inquires as follows.

Data Sampling: How to sample sensory data using smartphones in the regions of interest while not compromising the energy consumption and accuracy?

Data Processing: How to process the sampled data using smartphones in the regions of interest while dealing with considerable uncertainties in individual measurements?

Data Dissemination: How to disseminate the sampled and processed data using mainly smartphones while facing the intermittent end-to-end connectivity?

In order to answer above research questions, we shall discuss the corresponding problem definitions and hypothesises.

\section{Data Sampling with Non-deterministic Sensing Platforms}

Obtaining reliable and real-time data measured by onboard sensors is a daunting challenge. The bottom line is that smartphones are deliberately designed for communication, entertainment and daily experiences, but not for sensing tasks like ordinary sensor networks. Therefore, a sensing application should have as less impact as possible to the casual experiences of users, for example, battery life, performance speed and privacy. In particular, sampling data with non-deterministic sensing platforms faces with challenges as follows.

- Reliability: The intervals of sensor service callbacks, which return updated sensory values, vary and are not fully controllable. For example, the sampling rate of the accelerometers on most smartphones changes significantly between day and night, even during a short period because of energy conservation.

- Robustness: Some onboard sensors in smartphones such as microphones and cameras are exclusive-access resources. Application A will not be allowed to pull data from an exclusive sensor if application B is currently using it. In addition, an application process, its activities and its services, in general, can be terminated any time by the operating systems. Therefore, a smartphone cannot ensure that all sampled data will be delivered. 
- Real Time: Operating systems of smartphones such as Android set low priority for interacting with onboard sensors. In addition, smartphone OS is not a real-time os. Therefore, there are considerable delays in sensing, processing and sending data.

- Energy Efficiency: Data sampling and data transferring consume smartphone battery life significantly. The quick depletion of smartphones would deter many users from installing sensing applications on their smartphones.

To deal with the data sampling problems, we start from the following hypotheses:

Hypothesis 1. If a sensing application can piggyback on another application to acquire sensory data, it saves a lot of energy consumption while maintaining the performance, especially when many applications run simultaneously on many smartphones.

Hypothesis 2. Sampling data only around the moment when the interesting context changes saves energy consumption while still providing sufficient informative samples.

Based on these identified hypotheses, we propose efficient methods for data sampling that are partly presented in Chapter 4 and published in:

- V. D. Le, H. Scholten, and P. Havinga, "Flead: Online frequency likelihood estimation anomaly detection for mobile sensing," in Proceedings of the 2013 ACM Conference on Pervasive and Ubiquitous Computing Adjunct Publication, ser. UbiComp '13 Adjunct. New York, NY, USA: ACM, 2013, pp. 1159-1166.

- V.-D. Le, H. Scholten, and P. M. Havinga, "Online change detection for energy-efficient mobile crowdsensing," in Mobile Web Information Systems. Springer, 2014, pp. 1-16. (Best Paper Award)

\section{Data Processing with Non-deterministic Measurements}

It is hard to retrieve knowledge from measurements at a high quality level since sensory data measured by smartphones do not qualify as those of dedicated sensor platforms that are particularly designed for certain types of measurements. As a result, sensing applications based on smartphones have to face the large tolerance of measurements that significantly impacts the accuracy. The non-deterministic behavior of smartphones leads to the research questions as follows. 
- Statistic Modeling: Matching the distribution of measurements to a statistic model that can represent the measurement characteristics would facilitate the information retrieval given such data. Ideally mathematical analysis of measurements collected by non-deterministic devices is extremely difficult because of the uncertainty of errors, the insufficiency of the dataset, and the diversity of measurement conditions. A common question is how to simplify the assumptions about the measurement distribution so that it can be matched to a simple statistical model while it still is representative of the measurement characteristics.

- Cooperative Processing Bounds: In general, the deterioration in the accuracy of information retrieval can be reduced by collaborating the measurements among numerous smartphones observing the same event. However, how much the collaboration can improve the performance of nondeterministic devices needs to be thoroughly investigated. A lower bound or benchmark for estimation would provide insight into the effects.

- Anomaly-based Estimation: Smartphone-based sensing systems are considered as non-deterministic, albeit the great improvement of modern smartphones. Therefore, the research question is what technique is the most appropriate to remove the effects of jitters in measurements to improve the performance of the system.

To deal with the data processing problems, we start from Hypothesis 3 and 4:

Hypothesis 3. The quality of data sampled with smartphone platforms can be enhanced by aggregating the data from multiple smartphones in the neighborhood with regard to the same event.

Hypothesis 4. The whole distribution of data sampled with smartphone platforms can be expressed by some set of model parameters. Splitting the dataset into subsets would help to weed out the low quality measurements to improve the accuracy of the system since subsets with poor measurements do not fit the model.

Based on these hypotheses, we propose efficient methods for data processing that are partly presented in Chapter 5 and published in:

- D.V. Le, J.W. Kamminga, H. Scholten, and P.J.M. Havinga "Nondeterministic Sound Source Localization with Smartphones in Crowdsensing," in Pervasive Computing and Communications Workshops (PERCOM Workshops), 2016 IEEE International Conference on. IEEE, 2016. 
- D.V. Le, J.W. Kamminga, H. Scholten, and P.J.M. Havinga "Error Bounds of Localization with Noise Diversity," in Distributed Computing in Sensor Systems, 2016 IEEE International Conference on. IEEE, 2016.

\section{Data Dissemination with Intermittent Connectivity}

Short-range radio interfaces have been used as means of communication channels to gather and disseminate information in sensing networks. By means of the store-carry-forward paradigm, through WiFi or Bluetooth interface, a smartphone can receive, store, carry and send out messages to other smartphones if they are within communication range of each other. This approach can improve the robustness of systems, which still operate even when the infrastructure is damaged, either by natural catastrophe or sabotage. It also does not demand extra deployment costs. However, to be applicable for urban safety, smartphones have considerable performance problems in terms of delivery ratio, latency, and transmission cost.

These performance requirements can be met if the effects of following characteristics are reduced.

- Dynamics of Smartphones Movements: Since smartphones are carried by the users, the impact of human mobility on message delivery in HSNs is considerable. Furthermore, the movement patterns are hard to predict because of the unpredictable behavior and strict privacy of users.

- Diversity of Heterogeneous Sensor Networks: Smartphones themselves are already diverse from brand to brand, model to model, operating system to operating system. Moreover, there is much more diversity among smartphones and other HSN devices, for example, fixed-infrastructure sensors with Raspberry Pi. Such diversity results in a considerable effect on the system performance.

- Intermittency of Routing Paths: Utilizing the opportunities given by contacts among smartphones can deal with the lack of contemporaneous end-to-end connectivity in HSNs, though it is hard to predict the movement patterns. A good message routing scheme is an alternative to improve the delivery ratio, latency, and delivery cost.

To deal with the data dissemination problems, we start from the following hypotheses: 
Hypothesis 5. Each type of device in HSNs has its own characteristics with regard to the capability of delivering messages through the networks. Unifying different types of devices would provide better performance of message delivery than using only one type of device.

Hypothesis 6. Although precisely recognizing movement patterns of mobile devices is a hard problem, combining a store-carry-forward paradigm with historical data such as the user locations and contact times would improve data dissemination performance.

Based on these hypotheses, we propose efficient methods for data dissemination that are partly presented in Chapter 6 and published in:

- V.-D. Le, H. Scholten, and P. Havinga, "Evaluation of opportunistic routing algorithms on opportunistic mobile sensor networks with infrastructure assistance," International Journal On Advances in Networks and Services, vol. 5, no. 3 and 4, pp. 279-290, 2012.

- V.-D. Le, H. Scholten, and P. Havinga, "Towards opportunistic data dissemination in mobile phone sensor networks," in Proc. of The Eleventh International Conference on Networks (ICN 2012), 2012. (Best Paper Award)

- V.-D. Le, H. Scholten, and P. Havinga, "Unified routing for data dissemination in smart city networks," in Proc. of the 3rd International Conference on the Internet of Things (IoT2012), 2012.

- V. D. Le, H. Scholten, P. Havinga, and H. Ngo, "Location-based data dissemination with human mobility using online density estimation," in Consumer Communications and Networking Conference (CCNC), 2014 IEEE 11th. IEEE, 2014, pp. 450-457.

\subsection{Contributions}

The overall contributions of this dissertation are techniques and methods that deliver significant improvements in accuracy, robustness and energy efficiency for smartphone-based sensing applications in HSNs. These improvements were made possible through learning and exploiting opportunistic and cooperative approaches to deal with various challenges in data sampling, data processing, and data dissemination. We present four major contributions towards meeting the challenges to enhance performance of smartphone-based applications. 


\section{Contribution 1: Distributed Cooperative Architecture Support- ing Hybrid Sensing Paradigms}

We propose a symbiotic sensing paradigm with smartphones by exploiting the opportunities that enable balancing the tradeoff of the sensing burden on smartphones and their users. For example, environmental sound levels can be extracted from background of calling conversation. In fact, sensing is performed by utilizing an opportunity that meets the prerequisites of the sensing application. The opportunities include the smartphone context, user usage, user location, environment context, etc. In order to use the opportunities given by the emerging sensing capability and proliferation of modern smartphones, we incorporate the symbiotic sensing paradigm as well as existing sensing paradigms into a distributed and cooperative sensing framework. Our main contributions are as follows.

- We build evaluation models for sensing paradigms. The models are used to explore quantitatively the probability of success for each specific sensing systems. Our evaluation turns out that symbiotic sensing is suitable for large-scale sensing systems.

- We propose a cooperative distributed architecture that comprises three main components: data sampling, data processing and message disseminating.

Our research results will be described in Chapter 3, and have been published in:

- V.-D. Le, "Distributed opportunistic sensing in mobile phone sensor networks," in Pervasive Computing and Communications Workshops (PERCOM Workshops), 2013 IEEE International Conference on. IEEE, 2013, pp. 427428.

\section{Contribution 2: Cooperative Adaptive Sampling for Smartphone- Based Platforms}

Next, we focus on dealing with challenges of data sampling for smartphonebased sensing, especially sampling sensory data of power-hungry sensors such as microphones, cameras and Global Positioning System (GPS) modules. We show that conventional methods such as periodic sensing or random sensing 
are not practical for smartphone networks owing to the diversity and availability of sensing capabilities. For example, not all smartphones have a front camera or humidity sensor. Even if a smartphone has a front camera, it is not always available to capture images or videos for sensing applications, such as when the phone is in the user's pocket. Therefore, new sampling methods, probably based on the context of smartphones, need to be proposed. Our main contributions are as follows.

- We propose a cooperatively adaptive sampling approach that can significantly save smartphone and human resources while retaining high sensing performance.

- As our cooperatively adaptive sampling framework benefits from a lightweight technique to efficiently pinpoint the moment when the context meets the requirements, we bring forth an online change detection for energy-efficient sensing.

Our research results will be described in Chapter 4, and have been published in:

- V. D. Le, H. Scholten, and P. Havinga, "Flead: Online frequency likelihood estimation anomaly detection for mobile sensing," in Proceedings of the 2013 ACM Conference on Pervasive and Ubiquitous Computing Adjunct Publication, ser. UbiComp '13 Adjunct. New York, NY, USA: ACM, 2013, pp. 1159-1166.

- V.-D. Le, H. Scholten, and P. M. Havinga, "Online change detection for energy-efficient mobile crowdsensing," in Mobile Web Information Systems. Springer, 2014, pp. 1-16. (Best Paper Award)

\section{Contribution 3: Distributed Cooperative Data Processing for Smartphone-Based Platforms}

Towards addressing the challenges of smartphone-based sensing application in face of sensing diversity and availability, we first study their impact on sensing performance, particularly in terms of accuracy. Understanding the causality helps in finding the most suitable solution for the problem. Without loss of generality, we pick sound source localization using Android devices to evaluate the effects of errors on estimated locations. Although the cooperative and 
distributed data processing approach is evaluated on sound source localization, developing other sensing application based on our framework is similar and straightforward. Our main contributions are as follows.

- We analyze the time synchronization among smartphones through implementing a smartphone application to synchronise and measure the variance of the system clocks.

- We analyze the error of sensing latency that is counted from the moment a physical signal arrives at the sensor untill the moment its digital data is available in a buffer for reading.

- Based on these measurements and the impact on the localization application, we propose a new distributed approach based on a non-deterministic algorithm to localize acoustic sources.

- We evaluate our cooperative sensing on smartphone-based platforms with a real testbed experiment, cooperative sound source localization relying on only onboard microphones of Android devices.

Our research results will be described in Chapter 5, and have been published in:

- D.V. Le, J.W. Kamminga, H. Scholten, and P.J.M. Havinga "Nondeterministic Sound Source Localization with Smartphones in Crowdsensing," in Pervasive Computing and Communications Workshops (PERCOM Workshops), 2016 IEEE International Conference on. IEEE, 2016.

- D.V. Le, J.W. Kamminga, H. Scholten, and P.J.M. Havinga "Error Bounds of Localization with Noise Diversity," in Distributed Computing in Sensor Systems, 2016 IEEE International Conference on. IEEE, 2016.

\section{Contribution 4: Distributed Cooperative Message Dissemina- tion in Heterogeneous Sensor Networks}

Besides cooperative sensing, disseminating messages of detected events in a mobile network is important and challenging. With a store-carry-process-andforward paradigm, a message can be gradually sent to its destination. However, the paradigm relies heavily on the movement and willingness of users. To this end, we propose methods based on mathematical models and machine learning to overcome such challenges. Our main contributions are as follows. 
- We propose a movement model for pedestrians, cyclists, cars and buses in urban areas. We evaluate the new movement model with real datasets measured in the daily life of users. The evaluation results show that the new movement model is realistic and very close to the real dataset. This model enables realistic simulations for opportunistic mobile networks.

- Towards addressing the emerging Internet-of-Things, we propose a unified routing scheme for opportunistic and heterogeneous networks so that messages can be easily exchanged among different networks.

- In order to improve message routing performance in terms of scalability, we propose a location-based clustering routing for opportunistic mobile phone networks. The routing is based on machine learning techniques such as the online k-mean clustering to make the routing unobtrusive to users, lessen the burden on them.

Our research results will be described in Chapter 6, and have been published in:

- V.-D. Le, H. Scholten, and P. Havinga, "Evaluation of opportunistic routing algorithms on opportunistic mobile sensor networks with infrastructure assistance," International Journal On Advances in Networks and Services, vol. 5, no. 3 and 4, pp. 279-290, 2012.

- V.-D. Le, H. Scholten, and P. Havinga, "Towards opportunistic data dissemination in mobile phone sensor networks," in Proc. of The Eleventh International Conference on Networks (ICN 2012), 2012. (Best Paper Award)

- V.-D. Le, H. Scholten, and P. Havinga, "Unified routing for data dissemination in smart city networks," in Proc. of the 3rd International Conference on the Internet of Things (IoT2012), 2012.

- V. D. Le, H. Scholten, P. Havinga, and H. Ngo, "Location-based data dissemination with human mobility using online density estimation," in Consumer Communications and Networking Conference (CCNC), 2014 IEEE 11th. IEEE, 2014, pp. 450-457.

\subsection{Dissertation Organisation}

The remainder of this dissertation is organized as follows. Chapter 2 presents the state of the art of smartphone-based sensing systems and the open research 
questions. To answer the research questions, a cooperative distributed sensing architecture is presented in Chapter 3. Chapter 4, 5, and 6 present the solutions of data sampling, data processing, and data dissemination, respectively. Finally, Chapter 7 concludes this dissertation through re-summarizing the contributions and presenting future research directions.

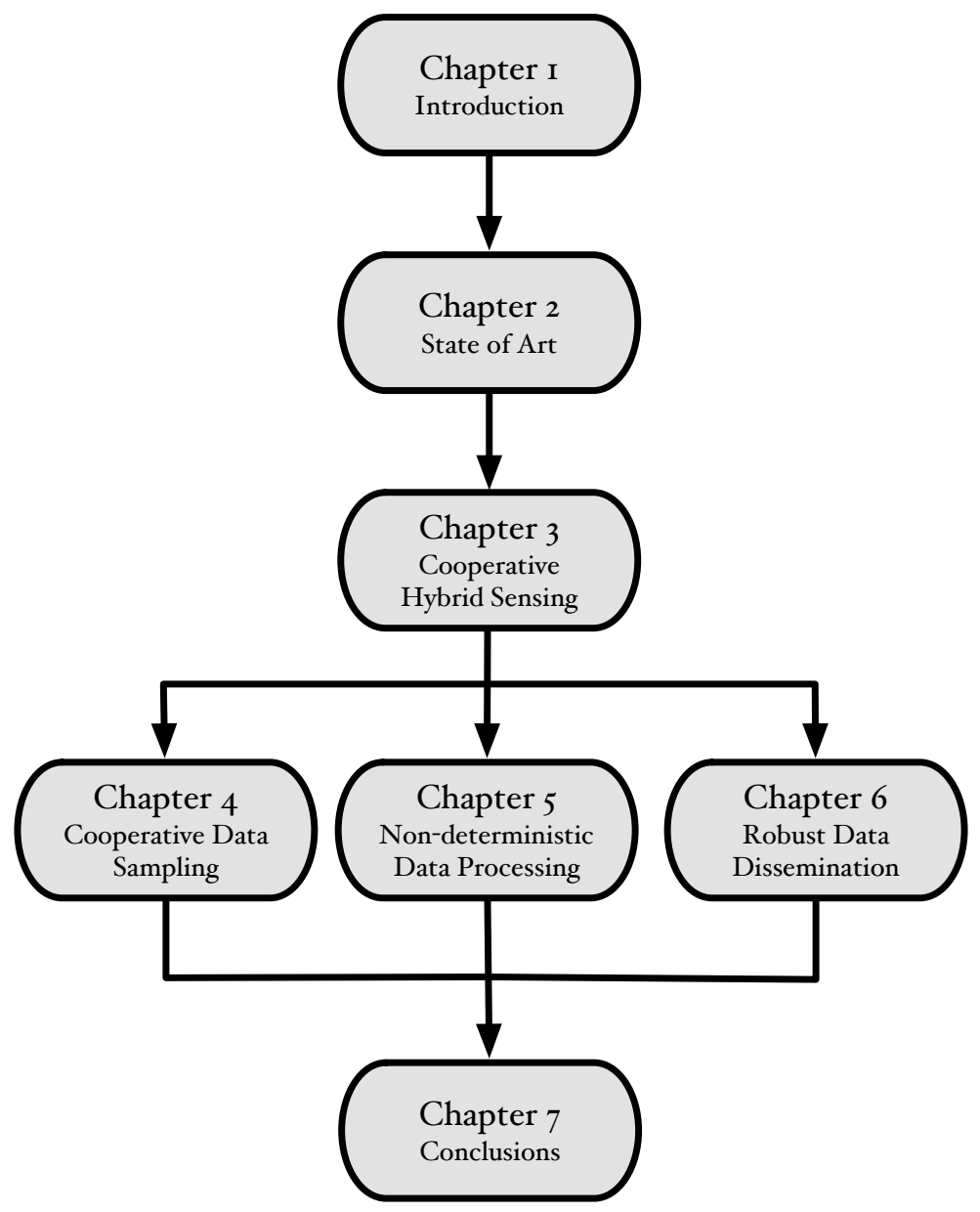

Figure 1.2: Dissertation organization. 


\section{State of the Art}

The advantages of the rapidly improved capabilities of smartphones have motivated researchers to create mobile phone sensor networks that can be applied in a wide range of human-centric applications, such as personal sensing, social behavior learning, environmental monitoring, and transportation. Starting from collecting data for processing offline at a primary data center, recent smartphones pave the way of cooperatively sampling and processing data online in a distributed manner. This chapter discusses the current state of the art of smartphone-based sensing through a number of categories. Based on the survey, a number of open issues and challenges are discussed.

\subsection{Introduction}

Forecast data from International Data Corporation (IDC) anticipate that there will have been 1436.5 millions worldwide smartphones shipped by the end of 2015. The amount is forecast to increase to 1902.3 millions by 2019 [4]. On the other hand, modern smartphones are integrated more and more powerful

This chapter is partially based on:

- V.-D. Le, H. Scholten, and P. Havinga, "Evaluation of opportunistic routing algorithms on opportunistic mobile sensor networks with infrastructure assistance," International Journal On Advances in Networks and Services, vol. 5, no. 3 and 4, pp. 279-290, 2012.

- V.-D. Le, "Distributed opportunistic sensing in mobile phone sensor networks," in Pervasive Computing and Communications Workshops (PERCOM Workshops), 2013 IEEE International Conference on. IEEE, 2013, pp. 427-428.

- V.-D. Le, "Towards opportunistic data dissemination in mobile phone sensor networks," in Proc. of The Eleventh International Conference on Networks (ICN 2012), 2012. (Best Paper Award, acceptance rate $31 \%$ ) 
technologies such as low-power sensors and communication interfaces. For example, the Samsung Galaxy S6 includes fingerprint, accelerometer, gyro, proximity, compass, barometer, heart rate and $\mathrm{SpO} 2$ sensors. It also has two quadcore CPUs, Quad-core 1.5 GHz Cortex-A53 and Quad-core 2.1 GHz CortexA57, and 3 GB RAM. Its battery can afford playing music up to 49 hours. The smartphones are also equipped most advanced network technologies such as LTE, WiFi 802.11 a/b/g/n/ac, dual-band, WiFi Direct, Bluetooth LE, NFC and infrared. The proliferation of smartphones as well as their emerging technologies has enabled researchers to develop even more diverse sensing applications than ever before, albeit not built specifically for sensing.

Although smartphones have been developed quickly in recent years, they still have limits on sensing capability when compared with dedicated sensor devices in the traditional Wireless Sensor Networks (WSNs), especially in terms of power consumption and accuracy. Most proposed smartphone-based sensing systems overcome the challenges by placing the burden on either the users in opportunistic sensing [1] or the smartphones in participatory sensing [2]. While these two strategies are quite effective in many sensing applications, they have not fully explored and exploited opportunities given by smartphones. Therefore, in this chapter we first present our survey on smartphonebased sensing systems. The survey is discussed with regard to a number of criteria including sensing paradigms, computing approaches, and communication networks. From the discussion, we conclude a range of open issues that should be targeted to improve the performance of smartphone-based sensing systems in Heterogenous Sensor Networks (HSNs).

The remainder of this chapter is organized as follows. In Section 2.2, we define a range of categories of techniques that have been used for smartphonebased sensing applications. Based on the categories, we survey a number of well-known sensing systems with regard to four common application domains in Section 2.3. Section 2.4 discusses a number of open research areas that we want to address in this dissertation. Section 2.5 summarises this chapter.

\subsection{Sensing Categories}

This section defines a number of sensing categories based on different criteria, such as the paradigm of sensing design, the computing approaches and the network types. 


\subsubsection{Sensing Paradigms}

In the design space, current sensing systems can be categorized into either opportunistic sensing or participatory sensing $[5,6]$. Opportunistic sensing collects data in an unobtrusive way such that the custodian might not be aware that the sensing application is running. Conversely, participatory sensing demands custodian involvement to collect and label data.

\section{Opportunistic Sensing}

Opportunistic sensing is a sensing mechanism collecting and processing data in an unobtrusive way, of which the custodian might not be aware. When the benefit brought by a sensing application is not personally appealing and hard to quantify, particularly with community sensing or sensing for scientific research, opportunistic sensing is preferred over participatory sensing. Opportunistic sensing aims to decrease the burden placed on the custodian, attract general users to let a sensing application running on their smartphones. In fact, the smartphone user may be even not aware of the active application. The sensing system automatically detects the context that meets application requests to perform sensing tasks. For example, the application detects if the smartphone is out of the pocket to measure the sound level to build a city noise map. In this way, the application does not require human intervention to actively and consciously participate in the sensing, enabling increasing the scalability of applications. However, opportunistic sensing is often difficult to develop, especially to solve the smartphone context problem [7]. Furthermore, since an application that is built based on the opportunistic sensing paradigm always try to detect the phone context, which is closely relevant to the custodian, the personally sensitive information may be leaked indirectly when providing the context, for instance, the location of the custodian.

\section{Participatory Sensing}

Participatory sensing, in contrast to opportunistic sensing, is a sensing mechanism demanding custodian involvement to collect data, label data, and/or give feedback. Lane et. al. described participatory sensing in [5] as a design that is suitable for sensing community. However, the participatory sensing paradigm also can be extended for other sensing types such as personal sensing, for example, labelling data or giving feedback for training and classifying personal activities. Therefore, a participatory system is deliberately designed as a sens- 
ing tool that requires the assistance of users to collect, search, publish, interpret and verify sensed information, as well as provide feedback for sensing loops. Consequently, participatory sensing places a burden on involved users (e.g pointing the camera of the smartphone to the sky and taking photos for air pollution monitoring) which costs time, money and efforts. It may disclose the custodian privacy too. To this end, a participatory sensing system is necessary to be able to gain enough interest of users in the smartphone sensing so that they are willing to be involved. A possible solution is to return some incentives to the participants [8].

To sum up, opportunistic sensing and participatory sensing are two extreme aspects of system design. One places the burden on smartphones and the other places the burden on users. Clearly it is necessary to understand the trade-offs. In addition, as more and more context-awareness and sensor are embedded into today smartphones, many later applications will require a hybrid design of both these sensing paradigms, or even a new sensing paradigm such as the one will be discussed in Chapter 3, which can mitigate phone context, custodian involvement as well as resource constraints.

\subsubsection{Smartphone-based Computing Approaches}

Five categories of smartphone-based computing approaches can be distinguished on the basis of two criteria. The criteria are where and how to compute the data. On the basic of the fist criterion, we can have three types of computing, namely centralized computing, local computing and cooperative computing.

- Centralized Computing: Data are first collected by smartphones and subsequently being sent to a dedicated server for processing.

- Local Computing: Collected data are locally and separately processed on each individual smartphone platform.

- Cooperative Computing: The data processing tasks are divided and shared among a group of smartphones. The smartphones cooperatively process the data with or without the assistance of a back-end server.

On the basic of the second criterion, we can distinguish online computing and offline computing.

- Online Computing: An online computing approach processes data elementby-element, serially without the need of having the entire dataset of the problem. 
- Offline Computing: An offline computing approach requires the entire dataset of the problem to be able to start with.

Note that some work may define online and offline computing are as what we define local and centralized computing, respectively. However, in the context of computing approaches, our definitions are more suitable.

\subsubsection{Smartphone Sensor Network Communication}

Smartphone networks are typically categorized into three following network types:

- Infrastructure Network: Smartphones communicates directly with access points such as WiFi routers or base stations of cellular networks, which are called infrastructure.

- Ad-hoc Network: A network formed by a group of smartphones that are connected through multi-hop without any infrastructure or "infrastructureless". Once a multi-hop path between two nodes are found, the path is supposed to be last for a long period.

- Delay/disruption Tolerant Network (DTN): Smartphones can be connected to each other via short-range radio interfaces such as Bluetooth and WiFidirect whenever they are in range. This sort of network is characterized by its lack of connectivity, resulting in a lack of instantaneous end-to-end paths.

\subsection{Smartphone-based Sensing Applications}

Smartphone-based sensing has been successfully applied to a wide range of applications. This section picks up some of successful instances that are close to human-centric sensing, which is an emerging domain nowadays. The selected smartphone-based sensing systems are listed in Table 2.1 along with categorized characteristics of the systems. 


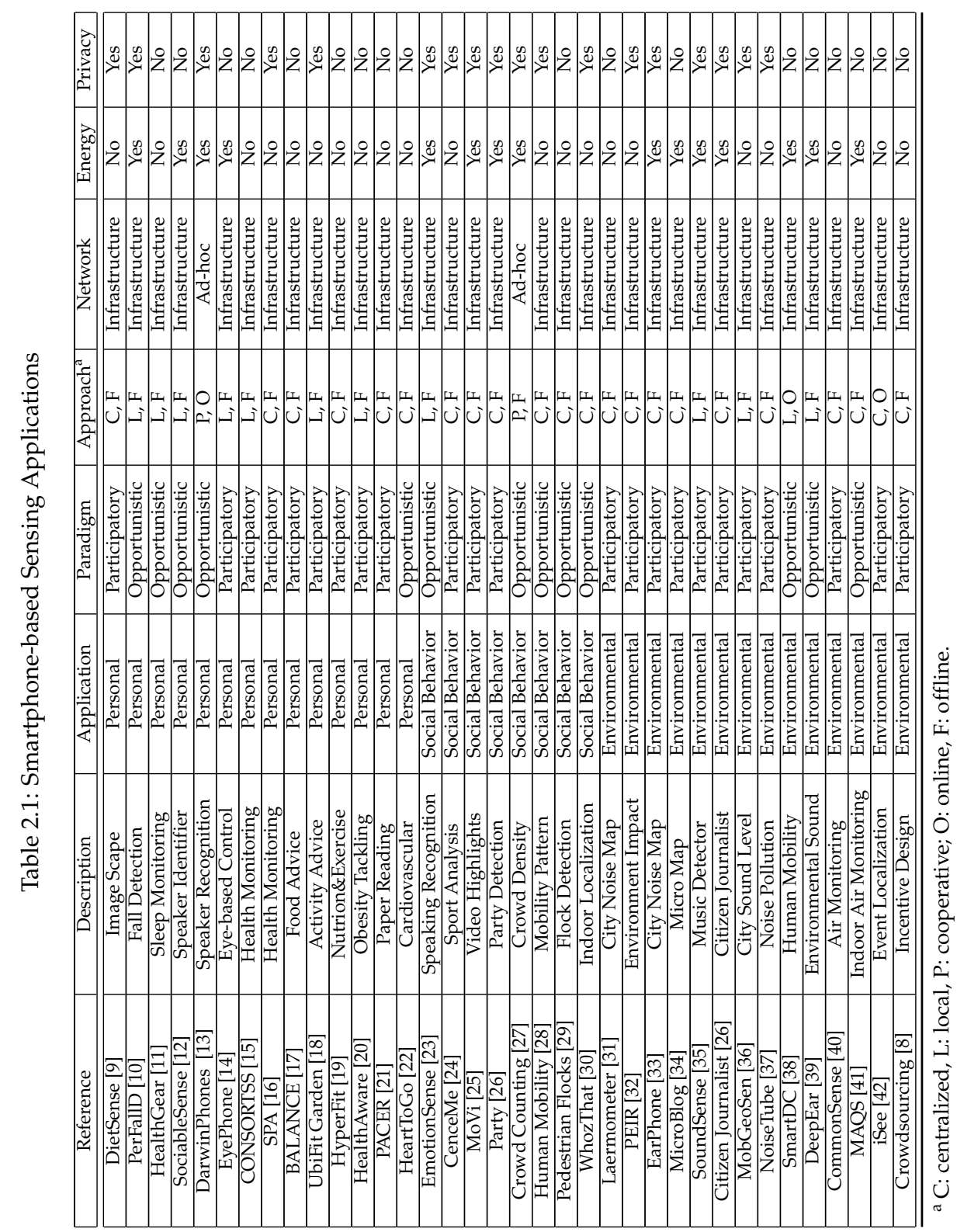



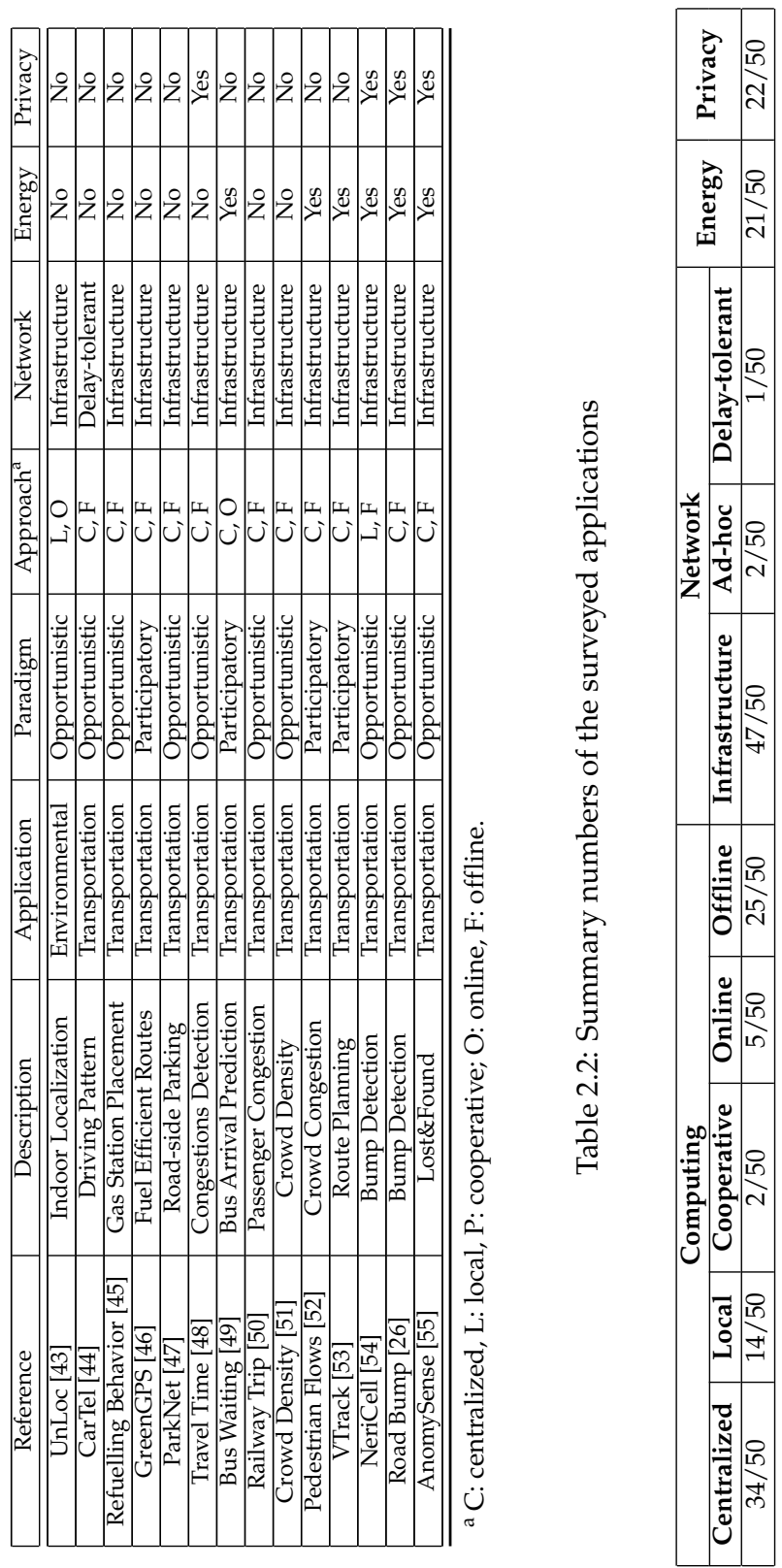


\subsubsection{Personal Sensing}

Personal sensing applications address collecting and analyzing data for a single individual, such as user's daily exercise, heart rate, blood pressure, sugar level, diary collection and emotion. Typically, data generated by a personal sensing application are preferably not shared with others. Although in applications that require sharing data such as healthcare, the data shared with medical doctors need to be also limited in order to preserve the user's privacy.

\subsubsection{Social Behavior Sensing}

We have discussed the probability of utilizing smartphones to recognize activity and location of individual users. Aggregating such individual information, the sensing system can obtain the behavior of a crowd or community as well as the social interactions among users.

\subsubsection{Environmental Sensing}

Although integrated sensors of smartphones are not as dedicated as ones in Wireless Sensor Networks, they outnumber the traditional sensor nodes. The proliferation and outnumbering sensors of smartphones are naturally suitable for large-scale environmental monitoring since there is no need of the sensor deployments and maintenance.

\subsubsection{Infrastructure Sensing}

The infrastructures such as road and traffic monitoring also have gained the interests from smartphone-bases system designers. Utilizing accelerometers and short-range radio interfaces (e.g., Bluetooth, WiFi Direct), we can develop a wide range of applications including detecting transportation mode, traffic accident control, road surface map, etc.

\subsection{Open Research Areas}

The results and analysis of surveyed articles hint at a number of open research areas, such as a hybrid sensing paradigm, a cooperative sensing framework, real-time data processing, data dissemination, energy-efficient sensing, and privacy protecting. 


\subsubsection{Hybrid Sensing Paradigm}

Previous work categorize sensing paradigms into participatory sensing and opportunistic sensing based on the criterion of the involvement of users in the sensing process. In participatory sensing, users actively collect or process data. In opportunistic sensing, the collecting and processing data are automatic, with minimal involvement of users. However, today smartphone sensing capability has enabled a broader range of diverse applications that are designed towards a distributed and cooperative manner. The applications requires to be optimized better in terms of smartphone-resource consumption and user-usage interference. With the new trends, it is likely that many new applications will need a hybrid sensing paradigm that can mitigate such requirements. An additional sensing paradigm is probably needed, besides participatory sensing and opportunistic sensing, to enhance the hybrid sensing.

\subsubsection{Cooperative Sensing Systems}

From Table 2.2 we observed that most previous smartphone-based sensing systems were designed as centralized, sending collected data to a back-end server for processing. The reason is probably due to the limited computing capability of old smartphones. As the number of smartphones has been increased insanely during last few years, the proliferation has led the communication issue on transferring a mass of raw data to a server. To deal with such problem, some sensing designs preferably extract features on smartphones then sent them to a back-end server for further processing instead of sending the raw data. Although this approach can temporarily solve the data transmission with current sensing demands and scale, which are relatively small, it will not be able to tolerate with the enormous amount of sensing devices in the emerging Internet of Things. Therefore, later sensing systems coped with such challenge by designing energy-efficient computing paving the way for pushing more computing load to local smartphones. However, the local computing approach faces a new problem due to the incomplete data set, which may significantly degrade the accuracy when compared to the centralized approaches. To this end, the cooperative computing approach would be the best suited. In particular, exchanging sensing data among a cluster of smartphones that together observe the same event will provide more insights of the unknown information. In addition, cooperative computing distributes the burden of computing on the smartphones to avoid heavily burning resources on a smartphone as well as improving computing reliability. Nevertheless, Table 2.2 sensing systems de- 
signed with cooperative computing are still very limited, only 2 over 50 surveyed articles.

\subsubsection{Online Learning Algorithms}

While most current approaches use either online or offline computing scheme, a complex real-time event processing may include both elements of online and offline computing. Generally speaking, real-time computing is frequently seen as online computing. However, an online algorithm may be updating based on a complete dataset that has been collected during a training phase. By definition of the offline processing in Section 2.2.2, this is offline computing. Moreover, if the offline algorithm that can test new input data based on an offline trained model fast enough, the result can be updated in near real-time. Combining both online and offline algorithm also enables optimizing resource consumption. Heavy processing is preferably computed offline while light-weight one is preferably computed online.

\subsubsection{Timely Data Processing}

We envision that future sensing applications will have to focus more on decision support and resource consumption to compete with each other to gain user interests. The goal of most smartphone-based systems will be shifted to gain an advantage over an opponent by having a shorter feedback loop in time. The goal to have a shorter feedback loop than other competing applications is necessary not only for critical application such as public safety and emergency response but also for routine sensing application such as activity recognition. A person jogging would like to know his or her conditions (e.g. speed, heart rate, and consumed energy) as quickly as possible to adjust his or her training.

In short, a future sensing application needs to minimize the feedback loop and resource consumption, which may involve either the online algorithm, the offline algorithm or both of them.

\subsubsection{Data Gathering}

To the best of our knowledge, as demonstrated in Table 2.1 and 2.2, most current smartphone-based sensing systems use Managed (Infrastructure) Wireless Networks such as Cellular Networks, mobile Internet, and WiFi hotspots to transmit collected data. In the near future, the amount of data generated by Internet of Things will exceed the bandwidth capability, albeit improvement of 
communication. In addition, it is necessary for sensing network systems of the future to be robust even if the sustainable infrastructures down due to catastrophes, disasters or sabotage. Among available solutions, using short-range radio interfaces, such as Bluetooth, WiFi Direct, and NFC to disseminate data among devices in an ad-hoc manner is one of the best solutions. The ad-hoc network operates based on the store-carry-forward paradigm. However, in our survey database, only 3 over 50 systems used the smartphone ad-hoc network or DTN protocols to transferring data. Therefore, data or message dissemination using the DTN protocols are very promising in near future sensing systems. It also results in new challenges in terms of delivery ratio, latency, and transmission cost since the links among nodes are disconnected most of the time.

\subsubsection{Energy Efficiency}

Reducing energy consumption was always an important issue in wireless sensor networks. With the rise of smartphones and cooperative computing, the problem is getting more important since battery is a main concern for most smartphone's users. Nevertheless, not many work in our survey, which is summarized in Table 2.1 and 2.2, discuss about energy efficiency. Roughly a half of the surveyed papers consider power consumption when designing their sensing systems. Even though, many of these work just stop at measuring the power consumption to evaluate their sensing systems. Only a few of them really try to optimize their sensing systems in terms of energy efficiency. Therefore, designing an energy-efficient algorithm for smartphone-based sensing applications are still very open, especially with cooperative approaches and continuous sensing.

\subsubsection{Privacy Protection}

Since smartphones are sort of people-oriented device, users hesitate because their privacy may be leaked through sensed data collected by their smartphones. In particular, smartphones can provide information about custodian's location, activities, emotion, social interactions, etc. Although some sensing systems try to remove the identity of users, the anonymity still can be breached as smartphones are physically close to their users and often connected to the global network. In general, participatory sensing is more vulnerable to privacy attack than opportunistic sensing because users have to participate in sensing tasks. However, the privacy of nonparticipating individual such as in opportunistic sensing also can be hacked through anticipatory schemes. Neverthe- 
less, more than half of surveyed systems listed in Table 2.1 do not mention about the custodian privacy at all. Even among the rest article which marked as "YES" in the table, only a few try to integrate a privacy mechanism into their systems, the rest just barely assume that the users have to trust the system or give the users the right to decide whether the privacy data will be shared or not.

\subsection{Summary}

In this chapter, we have reviewed state-of-the-art techniques and systems designed for smartphone-based sensing applications. Our study shows that most developed systems are centralized, offline and not real-time. Communication channels among smartphones mainly rely on infrastructure. In addition, energy efficiency is not studied well, albeit have been discussed. Throughout the remaining chapters in this dissertation, we address these open research areas to improve the performance of the sensing systems by focusing on exploring and exploiting the opportunities given by smartphones. 


\section{CHAPTER 3}

\section{Cooperative Hybrid Sensing}

Sensing paradigms such as opportunistic sensing or participatory sensing have been used to deploy sensing applications on a large scale. However, the significantly growing number of sensing applications in recent years has made most smartphone users reluctant to allow their devices to perform sensing tasks mainly because of battery and privacy issues. Therefore, in this chapter we first we propose new variants of sensing paradigms that provide insight into various parameters including the number of smartphones, the number of applications, the amount of app-using time, and the role of users that effect the probability of successively performing sensing tasks. In addition, we propose a smartphone-based sensing architecture in a distributed cooperative manner. Using the architecture, sensing systems can be developed and deployed in a wide range of sensing applications including urban sensing and smart cities.

\subsection{Introduction}

This chapter first discusses symbiotic sensing, a bio-inspired sensing paradigm for energy-saving sensing systems that are built on smartphone-based platforms. Since the number of smartphone applications has increased significantly while the sensing resources on smartphones are limited, the symbiotic sensing approach addresses sharing the resources as well as outcomes among the applications like symbiosis among living beings in the natural world. We

This chapter is partially based on:

- V.-D. Le, “Distributed opportunistic sensing in mobile phone sensor networks," in Pervasive Computing and Communications Workshops (PERCOM Workshops), 2013 IEEE International Conference on. IEEE, 2013, pp. 427-428. 
also propose evaluation models for the new and the existing sensing paradigms. Through a quantitative evaluation of the models given surveyed data, we showed that symbiotic sensing is complementary to existing sensing approaches. We also analyzed pros and cons of smartphone-based systems using different approaches. Albeit application diversity should be considered, symbiotic sensing has a potential to be a better choice than opportunistic sensing at large scale, where there are enormous numbers of smartphones and applications. Although the results from the quantitative evaluation do compare the probabilities of success when using different approaches, building out these sensing techniques will provide more conclusive evidence into what are the best choices for a smartphone-based sensing system.

Next, we introduces the software architecture of our sensing systems. The architecture is designed to allow collaborating smartphones to process sensory data in a distributed manner. In particular, the architecture comprises of three main stacks: data sampling, data processing, and data dissemination. These stacks are not only linked to each other from bottom to top inside each smartphone, but also can be connected to stacks deployed in other smartphones through a wireless communication. In short, this design aims at supporting hybrid sensing paradigms as well as distributed cooperative sensing approaches. The architecture is also designed in a way that shortens the feedback loops of information retrieval to increase the acceptance rate of using the applications.

The remainder of this chapter is organized as follows. Section 3.2 describes the opportunistic and participatory sensing paradigms and proposes a new sensing paradigm, the so-called symbiotic sensing. In Section 3.3, we propose a distributed cooperative sensing architecture that enables the commensal sensing as well as the hybrid sensing paradigms.

\subsection{Symbiotic Sensing}

In this section, we first discuss the existing sensing strategies. After that, we introduce a new sensing strategy through two subsections. The former describes the symbiotic sensing paradigm. The latter analyzes the evaluation models of the sensing paradigms including symbiotic sensing, opportunistic sensing, participatory sensing, and hybrid sensing. 


\subsubsection{Sensing Paradigms}

Most current sensing systems are developed based on either participatory sensing [2] or opportunistic sensing [1]. Participatory sensing systems require users to actively participate in sensing data. Meanwhile, opportunistic sensing systems can run unobtrusively and the users may not be aware of that sensing applications are running on their smartphones. In other words, the burden of sensing places on either users or smartphones. These extreme sensing paradigms restrain utilizing fully sensing opportunities provided by users and smartphones. Recently, there is also a new trend of developing applications based on diverse criteria. For example, Bubble-Sensing [56] is a new paradigm for binding a sensing task to the physical world using smartphones. In fact, Bubble-Sensing is a hybrid sensing paradigm that considers places of interests as the sensing condition. Once a smartphone is present within a place of interests, sensing tasks will be submitted to the smartphone.

When designing an urban sensing system, its performance on a large scale is usually hard to estimate since it depends on multiples uncertain parameters (e.g., the number of smartphones, the number of applications). To deal with such daunting challenges. Lane et al. [5] discuss the use of the opportunistic and participatory sensing paradigms through their proposed evaluation models. In particular, they contrast the two endpoints of the spectrum of the human involvement: the participatory sensing paradigm involves actions of the users; the opportunistic sensing paradigm is unobtrusive to the users. They conclude that a system designed with an opportunistic sensing approach easily supports large-scale deployments.

However, these existing paradigms do not consider the fact that the number of applications that may not be able to concurrently access the same sensor since some sensors such as microphones of Android smartphones can only be used by one application at a moment. That means that if the number of applications and/or the quantity of running time increases, the success rates of systems designed with the existing sensing approaches will decrease. In fact, recent surveys from Statista $[57,58]$ show that the number of applications has increased exponentially, from a few thousands in 2008 to more than 3.5 million applications in 2015. Moreover, a report from Nielsen.com [3] reveals that the monthly time spent per person on smartphone applications has risen $63 \%$ in two years, from 15 minutes per day in 2012 to 25 minutes in 2014 . Note that the year 2008 is when Lane et al. [5] discussed the sensing abstractions and their evaluation models. This may be the reason why their models does not consider the proliferation of smartphones applications. To this end, these new 
phenomena indicate that existing sensing paradigms need to be modified.

\subsubsection{Motivation}

By definition, symbiosis refers to relationships between organism of different species that show an intimate association with each other. In a symbiotic relationship, it requires at least one of the participating species with a nutritional advantage, usually called the host. In general, symbiosis is categorized into three types depending on the nature of the relationship: parasitism, commensalism, and mutualism.

- Parasitism: This relationship is detrimental to the host because the parasite derives nourishment from the host. In fact, the parasite cannot live without the host. Examples include ticks, fleas, and leeches.

- Commensalism: In this relationship, neither species is dependent on the other for its existence. However, only one of the partners benefits from the association. Examples include the relationships between porcelain anemone crabs and anemones. The crabs benefit by gaining protection from the anemones.

- Mutualism: In this relationship, both partners benefit from each other but may still be able to live an independent life. For example, the clownfish gain protection from the anemones. In return, they drop scraps of food for the anemones to eat.

In the context of the above symbiotic relationships, opportunistic sensing and participatory sensing are kinds of parasitism. In opportunistic sensing, the sensing application drains the sensing resources from the smartphones, which may be used by another application. Consequently, the smartphone battery is depleted more quickly and the other applications may be corrupted. In participatory sensing, the sensing application sends requests to the user to obtain the required data. The user is supposed to manually feedback his/her observation or activate the required sensors to collecting the data. Either way, participatory sensing demands effort from the users.

Conversely, if a sensing application is developed in a commensalism and mutualism-like manner, the application can benefit from the smartphones, other applications, and users without interrupting or draining many extra resources from them. For example, an application aiming to detect the mood of users can benefit from the chatting voice during a phone call made by the users. By 
doing so, the application consumes very little extra power consumption of the microphones and the smartphone. It also does not require the user to perform an extra task for sensing. More importantly, the sensing application can perform its sensing tasks without conflicts with the calling application. At the higher level of data processing, a location tracking application might want to use results provided by an activity recognition application to know if the user is walking down a stair to improve the tracking results. In return, the activity recognition application can utilize the user location provided by the location tracking application to improve the accuracy. For example, it is very unlikely that a person is running when he or she is inside a car.

Note that the sharing does not only happen within a smartphone but also among smartphones in a cooperative manner. An application also can acquire sensors or sensed information from other neighboring smartphones in a way that is similar to commensalism and mutualism to avoid conflict and save energy consumption.

\subsubsection{Description}

Inspired by aforementioned symbiotic relationships, we propose symbiotic sensing paradigms to enable sensing applications to be deployed on a large scale with numerous smartphones. Sensing tasks are carried out in a cooperative manner, i.e. a sensing task is accomplished only if it is performed by at least a certain of smartphones. In particular, we propose two paradigms of symbiotic sensing according to how the sensing resources are shared between the sensing application and other applications.

Definition 3.1. Commensal sensing is a sensing paradigm that allows sensing applications to piggyback on other applications, namely the hosts, to utilize available resources or retrieved information. Applications designed with commensal sensing do not detriment the hosts, but they benefit by gaining the off-the-shelf resources and results from the hosts. Note that ONLY the sensing applications benefit from the association.

In commensal sensing, as sensing applications reuse data or information obtained by host applications, energy consumption can be reduced. Furthermore, as the resources are shared, the conflict of simultaneous acquisition is avoided. For example, an application aiming to recognize the context of the 
surrounding environment can piggyback the phone call and photography applications when users make a phone call and take a photo, respectively. By doing so, the context-awareness application utilizes the microphones and cameras that are in used by other applications to reduce extra energy consumption.

Definition 3.2. Mutual sensing is a sensing paradigm that allows sensing applications to share either resources or results with each other to avoid acquiring or processing the sensory data multiple times. Applications designed with mutual sensing do not detriment each other, but they benefit by gaining the shared resources and results. Note that ALL applications that participate in the paradigm benefit from the association.

Mutual sensing requires a compromise between at least two sensing applications. Each application agrees to publish its retrieved information. In return, it will gain the retrieved information from the other application. For example, a location tracking application may want to use results provided by an activity recognition application to know if the user is walking up/down a stair to update the floor level of user, which is difficult to be recognized from location information only. In return, the tracking application provides the location context for the activity recognition application. For instance, it is very unlikely that a person is running when he or she is moving at a speed of cars on a highway.

In principle, commensal sensing and mutual sensing are similar from sensing application viewpoint. In either paradigm, a sensing application piggybacks on others to save the energy consumption and avoid conflict of occupying sensing resources. The only difference between them is how the sensing resources are shared. Therefore we use symbiotic sensing to refer to both commensal and mutual sensing, to distinguish them from opportunistic sensing and participatory sensing.

Figure 3.1 illustrates a common architecture of the symbiotic sensing paradigm. The key feature is a cross-application layer that allows applications to share sensed information such as contextual data, retrieved information and models. Unlike the opportunistic sensing paradigm, which perform sampling data only when the sensor is unoccupied, the symbiotic sensing acquires the sensor if and only if it is being used by another application to save energy consumption. This piggybacking behavior of symbiotic sensing is also applied for communication and retrieved information. For example, an activity recognition application may piggyback on a tracking application to enhance its reliability. It is unlikely that a smartphone user is walking when the tracking 


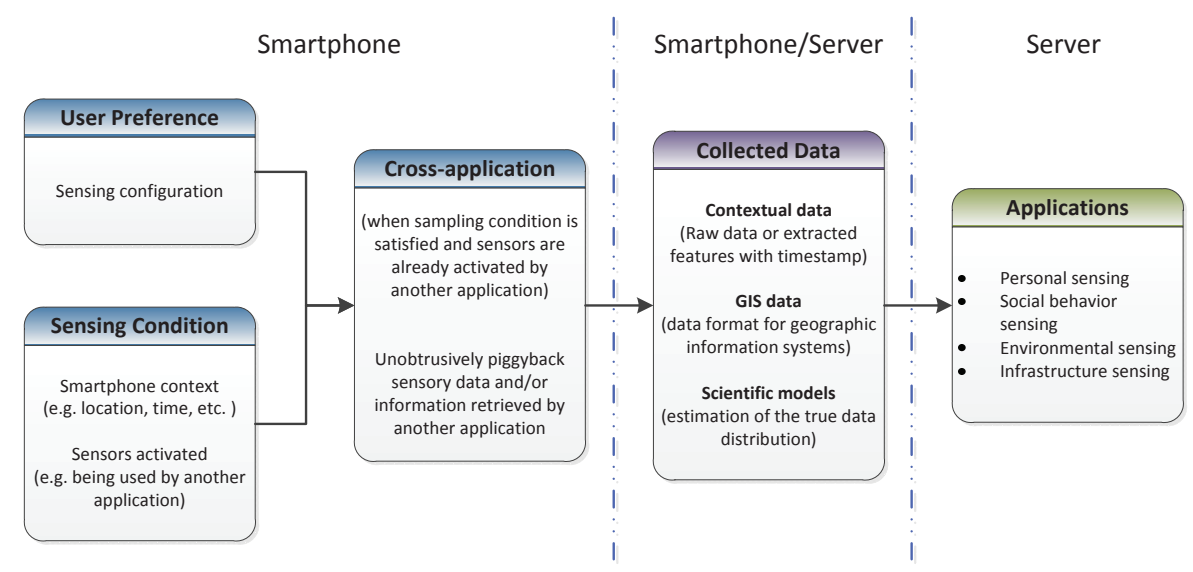

Figure 3.1: Common architecture for symbiotic sensing.

application tells that he/she is moving at a high speed similar to a car.

There are three key features as follows that make symbiotic sensing more scalable and energy saving to cope with the emerging popular of sensing applications.

- Applications designed with such sensing techniques can share same exclusive resources such as cameras and microphones with each other. Hence, the applications can coexist and run simultaneously without conflict.

- The applications can benefit each other by sharing their retrieved information so that it does not need to be processed multiple times.

- Mechanism is distributed and deployed through instances of applications on smartphones.

\subsubsection{Problem Formulation}

A typical smartphone-based sensing problem for urban sensing applications is to collect data in a region of interest by using off-the-shelf smartphone sensors. The data can be environmental noise, temperature, dust particles, carbon dioxide levels, etc. We assume that data is required to be collected from $C$ types of sensors. We further assume that the sensing application is installed on $N$ smartphones. Whenever a smartphone satisfies predefined sensing conditions, 
such as its location, time, physical position, and orientation, the application automatically performs the sampling task or requests the smartphone's custodian to collect data. The collected data will then be sent to a central server in raw or processed format. To obtain a certain accuracy, each type of sensory data needs to be collected by at least $M$ smartphones, where $M \leqslant N$.

To be able to choose a suitable sensing paradigm to deploy such sensing application, we need quantitative evaluations to compare the performance of sensing paradigms. Two most important aspects to evaluate a sensing paradigm is the probability of success and energy consumption. Therefore, we demonstrate the evaluation of the sensing paradigms on these aspects. Formally, we define success probability as follows.

Definition 3.3. Probability of Success of a sensing paradigm is the probability of that a sensing task is performed by at least $M$ smartphones to obtain a certain level of accuracy, given a system consisting of $N$ smartphones, $M \leqslant N$.

Let $p$ be the probability that the sensing application can perform a sensing task on a single smartphone, the probability of success of a generic sensing paradigm is given in the following lemma.

Lemma 3.1. Probability of Success

The success probability of a sensing paradigm is computed as:

$$
P=\sum_{k=M}^{N} C_{N}^{k} p^{k}(1-p)^{N-k},
$$

where $C_{N}^{k}=N ! /(k !(N-k) !)$ is the number of $k$-combination of $N$ elements.

Proof. The probability that the sensing task is performed by an arbitrary set of $k$ smartphones is the joint probability of that $k$ smartphones perform the sensing task and $N-k$ smartphones do not perform the sensing task, i.e. $p^{k}(1-p)^{N-k}$. In fact, there are $C_{N}^{k}$ combinations satisfying that the sensing task have to be carried out by $k$ smartphones. Therefore, the probability of that the sensing task is performed by $k$ smartphones is given by

$$
P(k)=C_{N}^{k} p^{k}(1-p)^{N-k} .
$$

Since the sensing requirement is that the sensing task needs to be performed 
by at least $M$ smartphones, the probability of success is given by:

$$
P=\sum_{k=M}^{N} C_{N}^{k} p^{k}(1-p)^{N-k} .
$$

Lemma 3.1 shows that the probability of success heavily depends on the probability that the smartphone can perform a sensing task that is required by the sensing application. By definition, sensing paradigms provide different sensing probabilities. Therefore, the probability of success for each kind of sensing paradigm is also different.

\subsubsection{Evaluation Model}

In this section, we derive quantitative models to evaluate sensing paradigms used as schemes for sensing applications. Note that the probability of success defined in [5] is under the assumption of that the required sensors are always ready to sample data as long as the smartphone owns them. However, under traditional sensing paradigms (participatory and opportunistic), the sensors might be unavailable when they are being occupied by another application. For example, the microphone cannot be used for sensing purpose when the phone is making a call, or the camera is unavailable during a video conversation. On the other hand, symbiotic sensing can avoid this conflict by sharing the sensing resource. Hence, we propose different formulas to represent the probability of success and energy consumption for each sensing paradigm. Since the probability of successfully collecting all $C$ types of sensory data is simply the joint probability of collecting each type of sensory data, for clarity and reading convenience, we will derive evaluation models for sensing applications that request only a sensor type, i.e. $C=1$. In particular, we present the derivation of Estimated Success Probability as follows.

As defined in Lemma 3.1, the probability of success is computed using the probability that a sensing task can be performed on a smartphone $p$. There are several issues that may affect the chance of a sensing task performed on a smartphone. For example, the user must agree to take part in the sensing task, the phone must be in a suitable context for data sampling (e.g. at a particular location), the phone must have the required sensor. Therefore, to make the derivation more clearly, we first define a number of elementary probabilities as follows. 
Definition 3.4. Probability of Permission $\left(P_{p}\right)$ is the probability that the user agrees to take part in sampling sensory data when there is a requirement query.

Definition 3.5. Probability of User $\left(P_{u}\right)$ is the probability that the user actually takes part in sampling data, for example, the probability that the user pulls his/her smartphone out of the pocket just to take a photo of the sky for an air monitoring application when the application requests.

Definition 3.6. Probability of Context $\left(P_{c}\right)$ is the probability that the smartphone has its context matched with the sampling conditions, for example, when the smartphone is out of the pocket for some other purposes so it can also be used to record environmental noise.

Definition 3.7. Probability of Sensor $\left(P_{s}\right)$ is the probability that the smartphone owns the sensor type required by the sensing task.

Definition 3.8. Probability of Occupation $\left(P_{o}\right)$ is the probability that the required sensors are being occupied by another application given the matched context, for example, the percentage of time the user uses his/her smartphone to take a picture for himself/herself.

For each sensing paradigm, the probability of a sensing task performed on a smartphone is different. For example, in opportunistic sensing, a sensing task can only be performed if the requested sensor is not in use by another application, whereas in symbiotic sensing, a sensing task is performed only if the sensor is collecting suitable data for other applications. Therefore, we define the probability of success for each sensing paradigm separately in the following theorems.

Theorem 3.1. Success Probability of Symbiotic Sensing:

The probability of success using a symbiotic strategy is given by:

$$
P_{b i o}=\sum_{k=M}^{N} C_{N}^{k}\left(P_{s} P_{c} P_{o}\right)^{k}\left(1-P_{s} P_{c} P_{o}\right)^{N-k},
$$

where $C_{N}^{k}=N ! /(k !(N-k) !)$ is the number of $k$-combination of $N$ elements.

Proof. Given the probability that the required sensor of the smartphone is occupied, $P_{o}$, the probability that the application can piggyback on such sensor is $P_{s} P_{c} P_{o}$, according to the definition of the joint probability. 
According to Lemma 3.1, we have the probability of success using a symbiotic sensing paradigm such that the sensing task can be performed by at least $M$ smartphones is given by:

$$
P_{b i o}=\sum_{k=M}^{N} C_{N}^{k}\left(P_{s} P_{c} P_{o}\right)^{k}\left(1-P_{s} P_{c} P_{o}\right)^{N-k} .
$$

Theorem 3.2. Success Probability of Opportunistic Sensing:

The probability of success using an opportunistic strategy is given by:

$$
P_{\text {opp }}=\sum_{k=M}^{N} C_{N}^{k}\left(P_{s} P_{c} \bar{P}_{o}\right)^{k}\left(1-P_{s} P_{c} \bar{P}_{o}\right)^{N-k}
$$

where $C_{N}^{k}=N ! /(k !(N-k) !)$ is the number of $k$-combination of $N$ elements and $\bar{P}_{o}=1-P_{o}$ is the complement of $P_{o}$.

Proof. Given the probability that the required sensor of the smartphone is occupied, $P_{o}$, the probability that the sensor is free is $\left(1-P_{o}\right)$. Therefore, the probability that the smartphone can execute the sensing task is $P_{s} P_{c}\left(1-P_{o}\right)$, according to the definition of the join probability.

According to Lemma 3.1, we have the probability of success using an opportunistic sensing paradigm such that the sensing task can be performed by at least $M$ smartphones is given by:

$$
P_{o p p}=\sum_{k=M}^{N} C_{N}^{k}\left(P_{s} P_{c} \bar{P}_{o}\right)^{k}\left(1-P_{s} P_{c} \bar{P}_{o}\right)^{N-k}
$$

Theorem 3.3. Success Probability of Participatory Sensing:

The probability of success using a participatory strategy is given by:

$$
P_{p a r}=\sum_{k=M}^{N} C_{N}^{k}\left(P_{p} P_{s}\left(P_{c} \bar{P}_{o}+\bar{P}_{c} P_{u}\right)\right)^{k}\left(1-P_{p} P_{s}\left(P_{c} \bar{P}_{o}+\bar{P}_{c} P_{u}\right)\right)^{N-k}
$$

where $C_{N}^{k}=N ! /(k !(N-k) !)$ is the number of $k$-combination of $N$ elements, $\bar{P}_{c}=$ $1-P_{c}$ is the complement of $P_{c}$, and $\bar{P}_{o}=1-P_{o}$ is the complement of $P_{o}$. 
Proof. Given the probability that the required sensor of the smartphone is occupied, $P_{o}$, the probability that the sensor is free is $\left(1-P_{o}\right)$. Therefore, the probability that the smartphone can execute the sensing task without the help of the user is the joint probability $P_{p} P_{s} P_{c}\left(1-P_{o}\right)$. If the sensing context is not matched, the application implemented with a participatory sensing scheme can request the user to help. However, the user might really do so with a probability of $P_{u}$. Therefore, the probability that the smartphone can execute the sensing task with the support from the user is the joint probability $P_{p} P_{s} \bar{P}_{c} P_{u}$. Thus, we have the probability that the smartphone can execute the sensing task with participator sensing is given by $P_{p} P_{s}\left(P_{c}\left(1-P_{o}\right)+\bar{P}_{c} P_{u}\right)$.

According to Lemma 3.1, we have the probability of success using a participatory sensing paradigm such that the sensing task can be performed by at least $M$ smartphones is given by:

$$
P_{\text {par }}=\sum_{k=M}^{N} C_{N}^{k}\left(P_{p} P_{s}\left(P_{c} \bar{P}_{o}+\bar{P}_{c} P_{u}\right)\right)^{k}\left(1-P_{p} P_{s}\left(P_{c} \bar{P}_{o}+\bar{P}_{c} P_{u}\right)\right)^{N-k} .
$$

Theorem 3.4. Success Probability of Hybrid Sensing:

The probability of success using a hybrid strategy is given by:

$$
P_{h y b}=\sum_{k=M}^{N} C_{N}^{k}\left(P_{p} P_{s}\left(P_{c}+\bar{P}_{c} P_{u}\right)\right)^{k}\left(1-P_{p} P_{s}\left(P_{c}+\bar{P}_{c} P_{u}\right)\right)^{N-k},
$$

where $C_{N}^{k}=N ! /(k !(N-k) !)$ is the number of $k$-combination of $N$ elements and $\bar{P}_{c}=1-P_{c}$ is the complement of $P_{c}$.

Proof. For a hybrid sensing paradigm, two possibilities exist: either the sensing context is matched or not. If the sensing context is matched, the smartphone will do sampling data regardless of the acquiring sensor is occupied or not. Therefore, the probability that the smartphone can execute the sensing task when the context is matched is $P_{p} P_{s} P_{c}$. If the sensing context is unmatched, the application will request the user to assist in sampling data. Therefore, the probability that the sensing task can be completed with the assistance of the user is $P_{p} P_{s} \bar{P}_{c} P_{u}$. Hence, the possibility that the smartphone can perform the sensing task is the joint probability $P_{p} P_{s}\left(P_{c}+\bar{P}_{c} P_{u}\right)$.

According to Lemma 3.1, we have the probability of success using a hybrid sensing paradigm such that the sensing task can be performed by at least $M$ 
smartphones is given by:

$$
P_{h y b}=\sum_{k=M}^{N} C_{N}^{k}\left(P_{p} P_{s}\left(P_{c}+\bar{P}_{c} P_{u}\right)\right)^{k}\left(1-P_{p} P_{s}\left(P_{c}+\bar{P}_{c} P_{u}\right)\right)^{N-k} .
$$

\subsubsection{Quantitative Evaluation}

In this section, we evaluate the symbiotic, opportunistic, participatory and hybrid sensing paradigms in terms of the models given in Section 3.2.5. We address a city mood application using smartphones carried by citizens. The application aims for helping citizens to understand the feeling of their city. For example, a street is angry as it is busy with a traffic jam or a central park is happy as it is having a festival with kids. To be able to infer the city mood, city sounds are primary sources that provide rich information. A conventional sensing system requires dedicated microphones densely deployed on a large scale. Such a system costs a lot of money and efforts to develop, deploy and maintain. On the other hand, the rise of smartphones has enabled utilizing onboard microphones of smartphones to collect data. Although there is a tension in terms of data quality, which is lower than that of dedicated sensing systems, the proliferation of smartphones can help improving the data quality in many situations.

In short, a sensing application is installed on a large number of smartphones. The application collects and processes the environmental sounds measured by the microphones of the smartphones. The sensing strategy is based on one of the sensing paradigms. In the context of such applications, we derive the model probabilities with realistic statistics.

\section{Parameter Settings}

It is likely that every single smartphone has at least one microphone, therefore, we set $P_{s}=1$ for the microphone sensor type. Since recording sound when the smartphone is in a pocket has dramatically low quality, we define when the smartphone is out of the pocket as the context matching. In 2012, Bristons spent average 90 minutes per day on their smartphones [59], of which approximate 14 minutes per day for using applications. We will use this analysis [59] as an approximate probability of the context matching. Thus we set $P_{c}=90 /(24 \times$ $60)=0.0625$. The survey [59] also shows that Bristons used their mobile phone 
$17 \%$ of such usage time for making phone calls. Because the microphone is an exclusive sensor that typically cannot be accessed by multiple applications at the same time except building some middleware platform for cross-sensor applications, we conservatively set $P_{o}=0.17$.

To derive $P_{p}$ and $P_{u}$, we conceptually map it to the probability of the first contact rates surveyed in a report on the feasibility of cell phone surveys [60]. In particular, the civilians were first called through their cell phones if they were willing to participate in a survey, in which they were expected to answer a list of interview questions. The study results show that 1561 out of 4448 agreed to participate into the interview. Therefore, we set $P_{p}=0.3$. The results also show that only 318 participant completed all the questions. That completion indeed requires some modification of planned behaviours conceptually relates to the probability of that a user participates in sampling data. Hence, we set $P_{u}=0.2$.

\section{Evaluation Results}

Given these probability values, the relationship between the derived values of success probabilities versus number of smartphones for symbiotic, opportunistic, participatory, and hybrid sensing paradigms are plotted in Fig. 3.2. In particular, Figure 3.2(a), (b), and (c) show the graphs of the success probabilities when the sensing application requires as least 1, 5, and 10 smartphones to collect data of the same event, respectively. Obviously, the hybrid sensing paradigm always has the highest probability of success regardless the values of the parameters. In this scenario, the opportunistic and participatory sensing paradigms have similar probability of success when varying number of smartphones. The symbiotic sensing paradigm has the lowest probability of success as it only utilises sensors occupied by other applications to save energy consumption, which is 0.17 . Nevertheless, when increasing the number of smartphones, the success probability values of the symbiotic paradigm also increase. This phenomenon confirms the hypothesis, the symbiotic sensing paradigm can perform as well as other sensing paradigms without consuming much extra resources if there are more smartphones collaborating.

Figure 3.2(a), (b), and (c) also show that the probabilities of success decrease when the sensing application requires more smartphones to acquire data of the same event. To maintain the probabilities of success, the application needs to be deployed on more smartphones. For example, to obtain a value of approximate 1 , the symbiotic sensing paradigm requires as least $N=500$ smartphones if $M=1$, while $N=1100$ smartphones if $M=10$. This phenomenon can be 


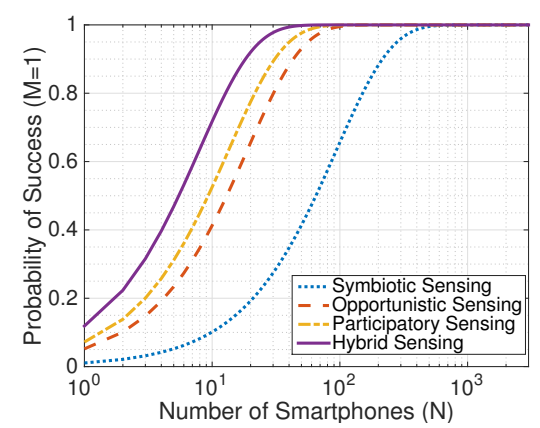

(a) $M=1$

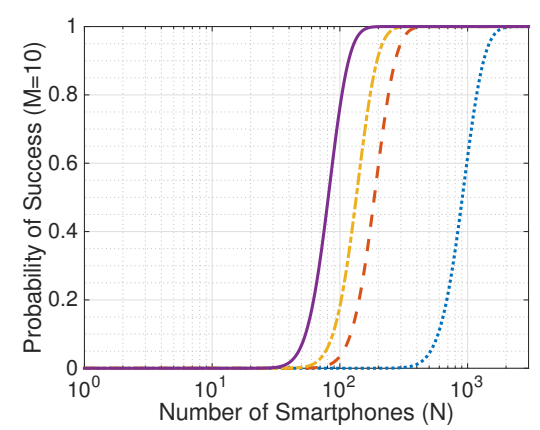

(c) $M=10$

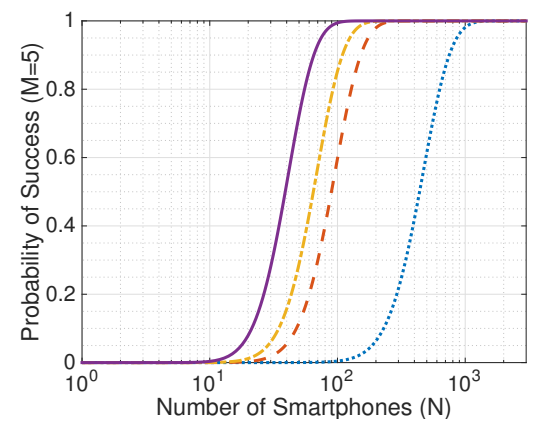

(b) $M=5$

Figure 3.2: The success probability of executing a noise measurement with citizens' smartphones for a city-sound-map application when using different sensing strategies.

theoretically proven because the probabilities of success are the sums of positive terms. The higher $M$, the smaller probabilities.

Since part of our work is developing symbiotic sensing to use the benefit of collaborations of a swarm of smartphones, it is interesting to analyze the relative merits and sensitivities versus number of smartphones and the probability of occupation for the symbiotic and opportunistic paradigms. Note that the participatory and hybrid sensing paradigms depend on not only the probability of occupation but also other probabilities. Therefore, we leave out the participatory and hybrid sensing paradigms for a fair and informative comparison.

Figure 3.3(a) and (b) show the success probabilities when the sensing ap- 


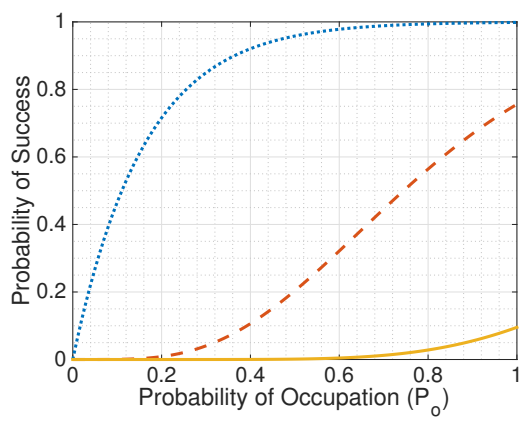

(a) Symbiotic

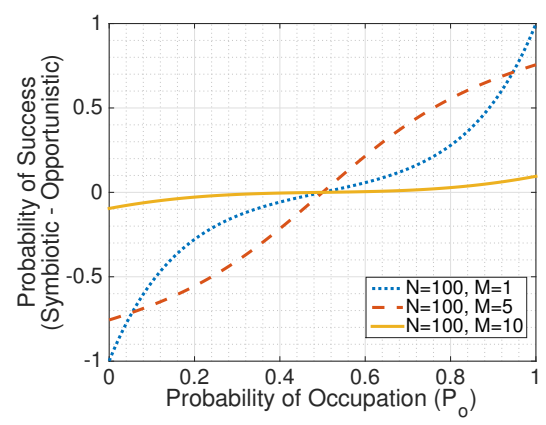

(c) Symbiotic - Opportunistic

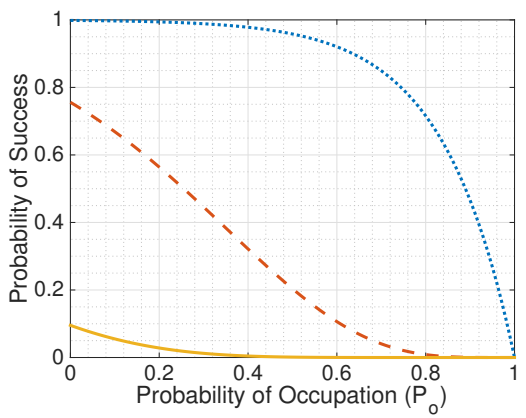

(b) Opportunistic

Figure 3.3: The success probability difference between the symbiotic and opportunistic sensing paradigms when varying the probability of occupation $P_{o}$ and $N=100$.

plication requires as least 1,5 , and 10 smartphones to collect data of the same event for symbiotic and opportunistic sensing, respectively. By definition, the symbiotic sensing paradigm is advantageous when $P_{o}$ is greater than 0.5 . The higher value of $P_{o}$, the more advantage. Fig. 3.3(c) plots the simple difference between the symbiotic and opportunistic sensing paradigms in terms of success probability against the probability of occupation $P_{o}$. In particular, the difference plotted in Fig. 3.3(c) is derived from the subtraction between the success probabilities of symbiotic and opportunistic sensing plotted in Figure 3.3(a) and (b), respectively.

The difference also varies with the number of smartphones to install the sensing application, $N$, and the number of smartphones required to acquire 


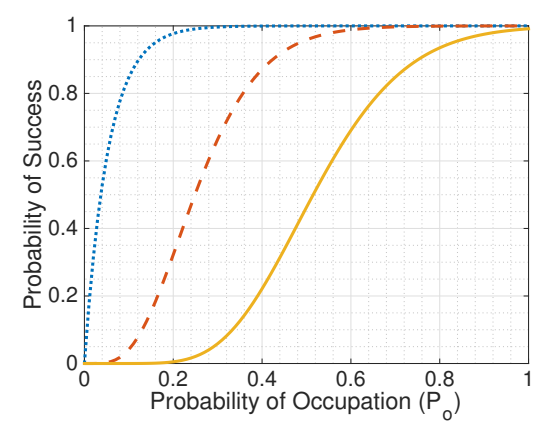

(a) Symbiotic

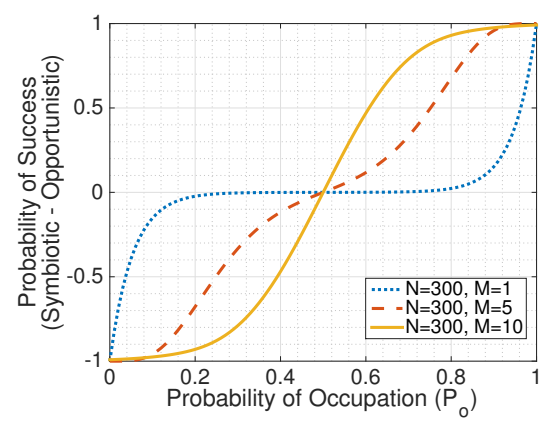

(c) Symbiotic - Opportunistic

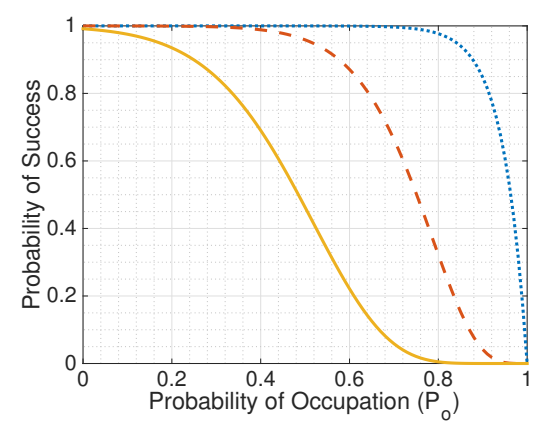

(b) Opportunistic

Figure 3.4: The success probability difference between the symbiotic and opportunistic sensing paradigms when varying the probability of occupation $P_{o}$ and $N=300$.

data of the same event, $M$. Figure 3.3 plots the probabilities of success when $N=100$. The graphs show that the symbiotic sensing paradigm gains more merits when the application requires fewer smartphones to sense the same event simultaneously. If the number of the required smartphones increases to above 10, the probabilities of symbiotic and opportunistic paradigms both get close to zero. Therefore, the difference is small.

When increasing the number of smartphones that installed the sensing application, symbiotic sensing gives more advantage if the application requires more smartphones to acquire data. Figure 3.4 shows that the difference increases when increasing the number of smartphones to 300 . The reason is that the success probabilities of both paradigms increases with the number of 


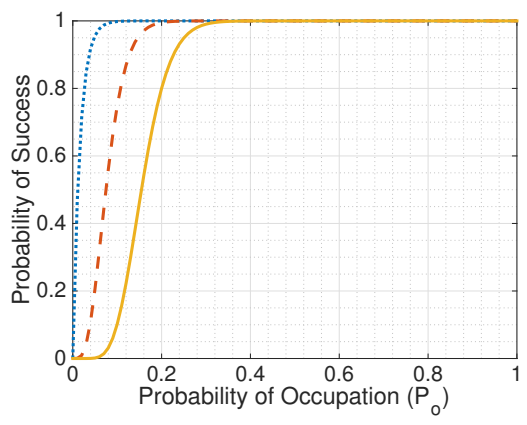

(a) Symbiotic

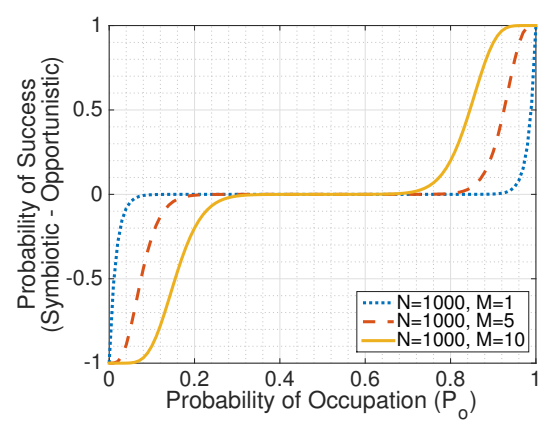

(c) Symbiotic - Opportunistic

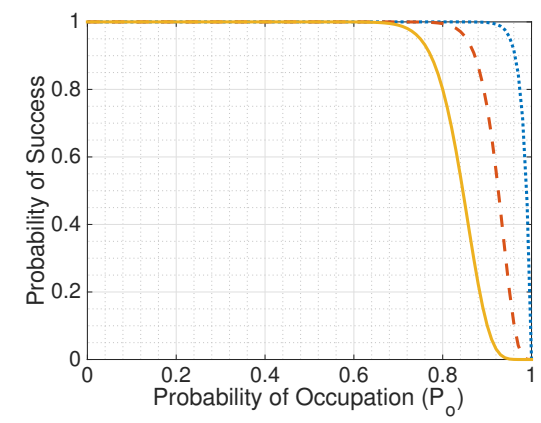

(b) Opportunistic

Figure 3.5: The success probability difference between the symbiotic and opportunistic sensing paradigms when varying the probability of occupation $P_{o}$ and $N=1000$.

smartphones, but toward the opposite sides of the balance point $P_{o}=0.5$.

However, when continuing to increase the number of smartphones, both paradigms approach the highest probability of success. It requires higher $P_{o}$ values to make use the advantage of the symbiotic sensing paradigm over the opportunistic sensing paradigm as shown in Figure 3.5. Note that a higher $P_{o}$ value means that more sensing applications deployed on smartphones acquire the same sensors, which would happen in the near future. In other words, the symbiotic sensing paradigm is more suitable to large scale sensing systems that require at least a number of smartphones to sense the same event. 


\subsection{Distributed Cooperative Sensing Architecture}

Since the main aim of this dissertation is to find suitable methods, techniques, and solutions for smartphone-based sensing systems, we introduce the software architecture that focuses on implementation on smartphones as depicted in Figure 3.6. Overall, the software architecture is designed in the way to enable cooperative sensing using the hybrid sensing approach. The software architecture consists of a number of components that can be categorized into three main stacks: data sampling, data processing, and data dissemination.

The data sampling stack function is to sense and collect information of events and then push the sensor data to the upper stack, data processing. The sampling is managed through a framework based on the hybrid sensing paradigm. To support opportunistic sensing, the smartphone context detector block is designed to notify the framework whenever the smartphone meets the required sensing condition. To support participatory sensing, a smartphone user can entry the interesting information through the user interaction block. Once the sensing condition is satisfied, the framework adaptively samples the events data through the cross-application sensor service block. Besides being able to adaptively sample data, the cross-application sensor block can also make it possible to share sampling information among applications to enable the symbiotic sensing approach. In addition, the sampling framework is cooperative. It can collaborate with the sampling frameworks implemented in nearby smartphones to share the sampling resources such as sensors.

The data processing stack is designed to cooperate with other smartphones to process the data sampled by the data sampling stack. It first gathers data from smartphones nearby then extracts features and infers the event. All these three steps are executed by the data fusion, feature extractor and estimator blocks, respectively. Note that these processes are distributed, communicate with others similar functional processes implemented in the other smartphones.

The results given by the estimator block need to be disseminated to civilians who are interested in the event information. This demand can be fulfilled by the data dissemination stack, in particular, the heterogeneous sensor networks. Heterogeneity means that the networks comprise various devices that can participate in sensing, transferring and receiving data. Examples are intelligent lampposts, smart vehicles and road-side WiFi units. Since we target at a low-cost, robust and reliable network, Delay/disruption Tolerant Network (DTN) protocols are preferred to disseminate event messages among the devices through short-range radio interfaces such as WiFi and Bluetooth. The bottom line is using the "store-carry-process-and-forward" paradigm. A device 


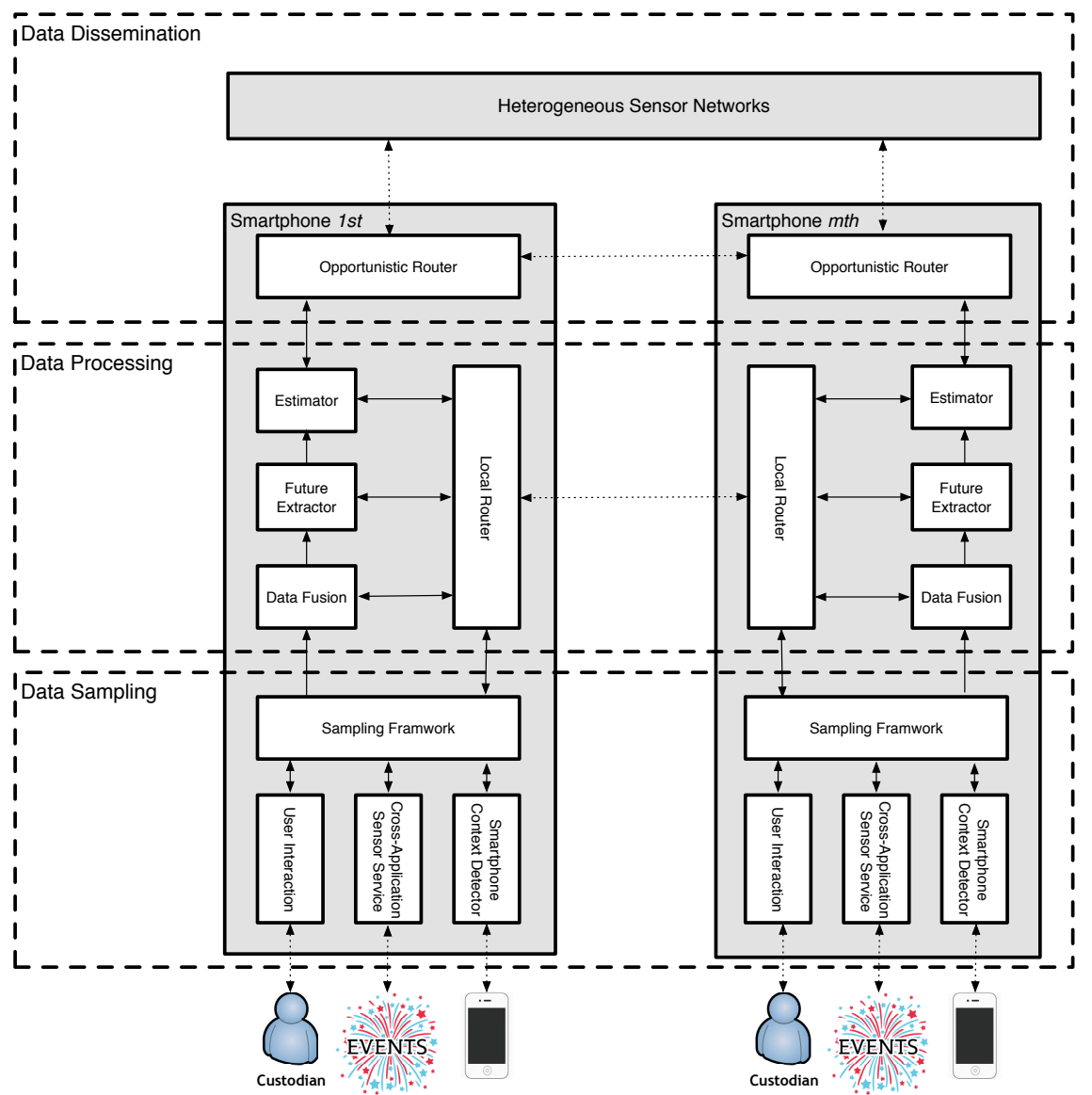

Figure 3.6: Software Architecture.

stores a message, processes the message, carries the message until the device is in range of communication of other devices and then forwards the message to the in-range devices. 


\subsection{Summary}

In this chapter, we have discussed and compared symbiotic sensing with other existing sensing paradigms such as opportunistic and participatory sensing. Opportunistic sensing executes sensing tasks unobtrusively to the users. Conversely, participatory sensing requires the users to use their smartphones to collect required data. However, the significantly growing number of sensing applications in recent years has made most smartphone users reluctant to allow their devices to perform sensing tasks mainly because of battery life. Exploring the symbiosis among sensing applications, we proposed symbiotic sensing that can save energy while maintaining as high performance as other existing paradigms. The key idea is that a sensing application should ulitize the sensory resources that are being used by another application, typically native applications of smartphones. By doing so, the new sensing paradigm collects required data with very little extra resource consumption. To be able to compare the symbiotic sensing paradigm with others, we presented mathematical evaluation models in terms of the success probability versus different parameters. The quantitative evaluation results given a real dataset indicate that symbiotic sensing performs better than others including opportunistic sensing in most situations, especially when having numerous smartphones and sensing applications. This is consistent with our hypothesis. The results also show that symbiotic approach is suitable to large-scale sensing systems that require multiple smartphones to sense the same event.

In addition, we presented a distributed cooperative architecture for smartphone sensing based on the concept of symbiotic sensing. The architecture is composed of three main layers, called stacks. The data sampling stack is addressed in Chapter 4. Chapter 5 deals with problems of the data processing stack. Finally, solutions for data dissemination are presented in Chapter 6. 



\section{Cooperative Data Sampling}

In Chapter 3, we have introduced the cooperative distributed sensing architecture that can support a hybrid sensing paradigm for event detection. In this chapter, we now expand upon the lowest stack, data sampling, exploring and exploiting the opportunities given by smartphones to provide confident and low-energy-consumption sampling in a distributed manner. In other words, we introduce a cooperative sampling scheme that can support multiple sensing paradigms as well as energy saving. Moreover, the scheme should allow sampling unfolding events as quickly as possible so that an application can support the user to make a decision faster and gain an advantage over the other applications.

\subsection{Introduction}

The fact that sensors are everywhere and are being designed into smartphones offers researchers a rich and powerful computing platform to develop sensing applications including urban safety, environment monitoring, activity recognition, and context awareness. Most recent approaches are based on either op-

This chapter is partially based on:

- V. D. Le, H. Scholten, and P. Havinga, "Flead: Online frequency likelihood estimation anomaly detection for mobile sensing," in Proceedings of the 2013 ACM Conference on Pervasive and Ubiquitous Computing Adjunct Publication, ser. UbiComp '13 Adjunct. New York, NY, USA: ACM, 2013, pp. 1159-1166.

- V.-D. Le, H. Scholten, and P. M. Havinga, "Online change detection for energy-efficient mobile crowdsensing," in Mobile Web Information Systems. Springer, 2014, pp. 1-16. (Best Paper Award) 
portunistic sensing or participatory sensing (see Table 2.2 and 2.1). However, the sensing capability of smartphones has significantly improved recently. This improvement enables a broader range of sensing approaches to be developed in a cooperative distributed manner. Therefore, we introduce a framework of cooperative distributed sampling that is able to support collecting data with various sensing paradigms such as opportunistic, participatory, commensal, and hybrid sensing. For the opportunistic sensing paradigm, the framework provides a module to detect when the context of a smartphone is suitable for collecting informative data, for example, when the smartphone is out of the pocket to measure the environmental noises. For the participatory sensing paradigm, the framework provides a user interface to send requests to the user to collect data, and to receive inputs from the user. For the commensal sensing approach, the framework provides a cross-application service that can share sensor resources among the applications. All such functionalities are able to work together to support a hybrid sensing approach, a combination of various sensing approaches.

In addition, the significant development of smartphone technologies and the demand of smartphone-based sensing have led to that more and more sophisticated and power-hungry sensors such as pressure sensors, humidity sensors, dust particle sensors, and gas sensors to be integrated. Consequently, data harvesting through mobile phones invokes a variety of challenges to overcome limited resources. Despite possessing higher battery capacity than conventional sensor devices do, smartphones consume much more power. It is also hard to persuade users to sacrifice battery power for sensing tasks. These resource constraints have deterred researchers from developing sensing applications running on smartphone platforms. Therefore, an effective scheme to adaptively sample sensor data is becoming more demanding than before, especially in continuous monitoring.

To cope with the energy constraint, a lot of work has been proposed such as [61-65]. Most of these works use machine learning techniques to estimate parameters of sensing context, for example, user mobility patterns, user mobility states and smartphone context. Since the sampling schedule is fixed after the training phase, these techniques are suitable for adaptive sensing applied to daily activities, however, in practice sensing context varies largely. In particular, smartphones carried by peoples have extremely dynamic movement patterns and environments. For example, a person may visit various places and do a lot of unpredictable activities during a day. An adaptively sampling technique that predicts incoming data based on a model learned by a machine learning algorithm may miss important data, especially when trying to detect 
short and sophisticated events. Existing techniques also requires a considerable delay to recognize whether the context matches with the sensing conditions.

Therefore, we enhance our the sampling framework so that it can efficiently save energy. In normal conditions, most sensors are switched off to conserve energy. Only a small set of sensors are turned on to monitor the sensing context. Once the sensing context is matched, the framework triggers requested sensors to collect the data. After a predefined period, the sensors are set back to the idle mode. As smartphones in a neighbourhood are connected, the framework in a smartphone can also invoke sensors from other nearby smartphones to enhance data collecting.

The remainder of this chapter is organized as follows. Section 4.2 points out current works that are most related to our adaptive sampling approach. In Section 4.3 we extend the software architecture of the data sampling stack. Next, we present an online adaptive sampling scheduling method that is suitable for measuring data in dynamic contexts with low power consumption devices. Section 4.5 presents a new technique to detect the change points of context statuses. Evaluation and experiment are carried out in Section 4.6. Finally, Section 4.7 concludes this chapter.

\subsection{Related Work}

There are collaborative adaptive sensing schemes based on a set of smartphones to reduce energy consumption. Darwin [13] is a collaborative reasoning framework built on three concepts: classifier/model evolution, model pooling, and collaborative inference. SocialeSense [12] controls the sampling rate while balancing the energy-accuracy-latency tradeoffs based on reinforcement learning mechanisms. Both schemes have a heavy computation load for smartphone platforms. The schemes check whether the measurement of a node can be predicted based on the measurement of neighbors. If so, this node will be turned into passive mode. This scheme will not be efficient in sparse networks, where most smartphones are usually not in the communication range of neighbors.

Two works that are technically close to our sampling framework are EmotionSense [61] and Jigsaw [65]. EmotionSense, a mobile phone based adaptive platform for experimental social psychology research, uses first order logic predicates to make rules to trigger sensors or change sampling rate. The rules can be defined by users, for example, starting the GPS receiver only if the user is currently moving. Jigsaw supports continuous sensing applications on mobile phones by comprising a set of sensing pipelines for the accelerometer and 
microphone to judiciously trigger the GPS sensor. The combination of such three sensor types takes into account the mobility and behavioral patterns of the user to reduce energy consumption. Even though both EmotionSense and Jigsaw greatly improve sensing performance in terms of energy cost, they are not suitable for sampling dynamic events. EmotionSense would have many false detections of changes because it requires a silent phase of streaming data to detect user emotion. In addition, both Emotion Sense and Jigsaw require to run feature extractions and classifiers given several data frames to be able to generate triggers. That causes a considerable delay of several seconds up to minutes as mentioned in their work. Within such delay, an emergency event probably had passed before the trigger was generated.

To the best of our knowledge, most adaptive sampling algorithms that periodically tune either sampling rates or subsets of sensor nodes cannot be applied to continuous sensing with mobile phones, especially for detecting short and non-deterministic events. In particular, the surrounding context varies largely since mobile phones carried by people have extremely dynamic movement patterns and environments. A person might visit various places and do a lot of unpredictable activities during a day. Adaptive sampling based on predicting incoming data with statistical models may miss important data, be ineffective, and suffer a considerable delay, especially in situations like accidents that happen unpredictably in a short time. Moreover, turning on power-hungry sensors even with low sampling rates will deplete the battery very quickly in a few hours or even less. To this end, we propose a new scheme that provides informative real-time sensor data, which can be used to retrieve the information of related events while optimizing the power consumption.

\subsection{Sampling Architecture}

The cooperative distributed sampling framework is composed of five main components as illustrated in Fig. 4.1: Sampling Management, Event States Detector, Cross-Application Sensor Service, User Interaction, and SmartphoneContext Detector. These five components are essential to enable hybrid sensing approaches to be deployed in a cooperative distributed manner.

\subsubsection{Cross-Application Sensor Service}

Sensors embedded on smartphones can be either exclusive or non-exclusive. If a sensor is exclusive, it cannot be simultaneously accessed by multiple ap- 


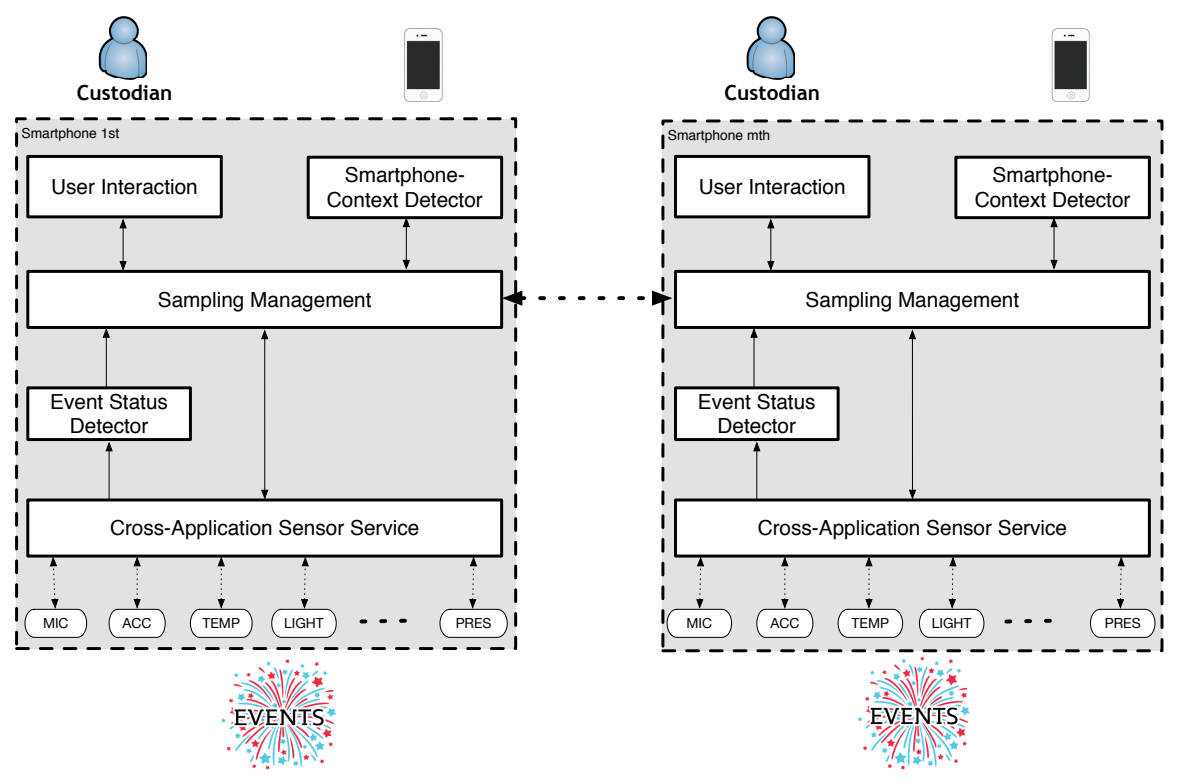

Figure 4.1: Sampling Architecture.

plications. Examples include microphones and cameras. To this end, CrossApplication Sensor Service is designed to enable sharing exclusive sensors among applications. As a result, the symbiotic sensing approach is more efficient since Cross-Application Sensor Service allows a sensing application to use the sensors that are currently in use by other applications.

In addition, each application may require a different sampling rate, which can be different from the native sampling rate. However, the native sampling rate is non-deterministic. It may vary depending on the states of the smartphone and the type of the smartphone, albeit with the same setting. CrossApplication Sensor Service aims at providing the expected sampling rate for each application by downsampling and upsampling.

Since sampling sensory data is typically power-hungry, Cross-Application Sensor Service is also designed to be able to switch on/off sensors according to the parameters received from Sampling Management. In general, powerhungry sensors are preferred to be idle if the event context looks normal and only turned on for a short period if Sampling Management sends a trigger that 
indicates some interesting event may happen and it is better to sample more intensively.

\subsubsection{Smartphone-Context Detector}

To save energy while adaptively sampling data, the framework should collect data if and only if the context of a smartphone meets the required condition. For example, a sensing system may be interested in events at certain places, therefore, the smartphone is required to start sampling data if and only if the smartphone reaches the areas of interests [56]. Smartphone-Context Detector is designed to detect when such conditions are satisfied.

Note that energy saving is a predominant requirement when designing techniques for smartphone-context detection. Therefore, the techniques should be either lightweight or rely on native services/broadcasters provided by the smartphone systems. In particular, a simple rule is applied to the context of a smartphone given sensory data. For example, the light sensor of a smartphone can detect correctly if the smartphone is inside a pocket in most of the situations. Another example is using the Global Positioning System (GPS) data to detect if the smartphone is at the places that are suitable for performing data sampling. The context of a smartphone can also be detected by listening to a native service. For instance, registering to the TelephonyManager class to listen when the smartphone is in a call with the Android platform.

\subsubsection{Event States Detector}

To avoid constantly sampling sensory data and save battery life, we have introduced Smartphone-Context Detector to enable sampling data only if the sensing condition is matched. In some cases, the sensing condition can be held for a long period. For example, a user may stay at an area of interest a whole day. Continuously collecting data while the user is still within the area of interest would consume a lot of smartphone battery too. Therefore, we design Event States Detector to enhance energy saving based on detecting the change points of event states. In fact, change points include anomalies and transitions of events. Our hypothesis is that only sensory data sampled around the change points are interesting and sufficient to monitor contexts. Therefore, it requires only a minimum set of sensors, namely proactive sensors, to continuously monitoring context changes. Other types of sensors, namely reactive sensors, are set idle to save power consumption and will be invoked to enhance sensing data 
after a change is detected. For example, by monitoring a light sensor, an algorithm gives a hint that something may be happening if there are fireworks and smartphone is out of the pocket. Then the algorithm will generate a trigger to enhance collecting data through additional sensors such as microphones to be able to detect the fireworks better.

The trigger is required to be as real-time as possible. In other words, a trigger has to be generated right after the event started, preferably few milliseconds at most, so that other sensors can be switched on fast enough to capture the event information. Note that continuously processing proactive sensory data to detect a change point is also a burden. Therefore, Event States Detector has to be lightweight, real-time, and reliable.

\subsubsection{User Interaction}

There also exist sensing systems that are built to request users of smartphones to give some input about an event that they are observing, which are categorised as participatory sensing. For example, [42] asks a smartphone's user nearby an event to swipe his/her smartphone's touchscreen towards the direction of the event. By doing so, the system can localize the position of the event, for instance, localizing areas of a fire in campuses and cities. User Interaction is designed to facilitate entering the observed data by the users.

A complex procedure likely makes the users refuse to participate in retrieving data. Therefore, User Interaction has to be designed in a way that requests the users to modify their behaviors as less as possible.

\subsubsection{Sampling Management}

Sampling Management is the central component that controls the sampling process. Based on the context information given by Smartphone-Context Detector, Event States Detector, and User Interaction, Sampling Management controls Cross-Application Sensor Service to collect required sensory data. Note that our data sampling stack is designed to support various sensing paradigms including opportunistic sensing, participatory sensing, commensal sensing, and hybrid sensing. Whenever the smartphone's context is matched with the sensing requirements, Smartphone-Context Detector will inform Sampling Management to invoke the sensors of the smartphone. This strategy is considered as Opportunistic Sensing. Sampling data also can be performed as a participatory sensing technique by requesting the users to give feedback on the event that he/she observed. 
In addition, a Sampling Management module on a smartphone can communicate with other Sampling Management modules on smartphones in the neighborhood to perform cooperative sampling. In particular, the trigger generated by a smartphone can be used to invoke additional sensors on nearby smartphones. Moreover, sensory data collected by these smartphones can be aggregated through a short-range radio to enrich the information about the interesting event. Furthermore, to improve energy saving, Event States Detector has to be implemented with a lightweight reliable change detection technique to accurately generate a real-time trigger whenever an interesting event changes its state.

\subsection{Minimum Active Duration Sensing Scheduling}

In this section, we describe a sensing scheduling method that is designed to reduce energy consumption based on the architecture described in Section 4.3. Even if the sensing conditions are matched, sensors should be switched to the idle mode as long as possible to save more energy consumption. The key idea of the sensing scheduling method is minimizing the durations of sensors in the active mode based on detected change points of context states while still being able to collect informative data.

\subsubsection{Change Points of Context States}

As change points might be misclassified with conventional anomalies or outliers in data, again, we emphasize that their definitions are different in the context of sensing. An anomaly in data is defined as an unexpected pattern in a dataset, which typically indicates some kind of problem, such as, a road bump, a gun shot or a defect of an engine. However, changes not only include such anomalies but also are referred to the transitions of a context, such as the sequential sounds in Fig. 4.2. Since data next to transitions like in Fig. 4.2 do not have significant dissimilarity in pattern with the previous one, a good anomaly detection technique might be ineffective under such circumstances, albeit the technique can perform well with the standard anomalies.

Most smartphone-based sensing applications require continuous sensing, which is the burden on the battery. In fact, many of these applications are only interested in data collected around change points or anomalies. For example, knowing when the user starts and stops walking is more than enough in many activity monitoring applications. Therefore, an adaptive scheduling method 


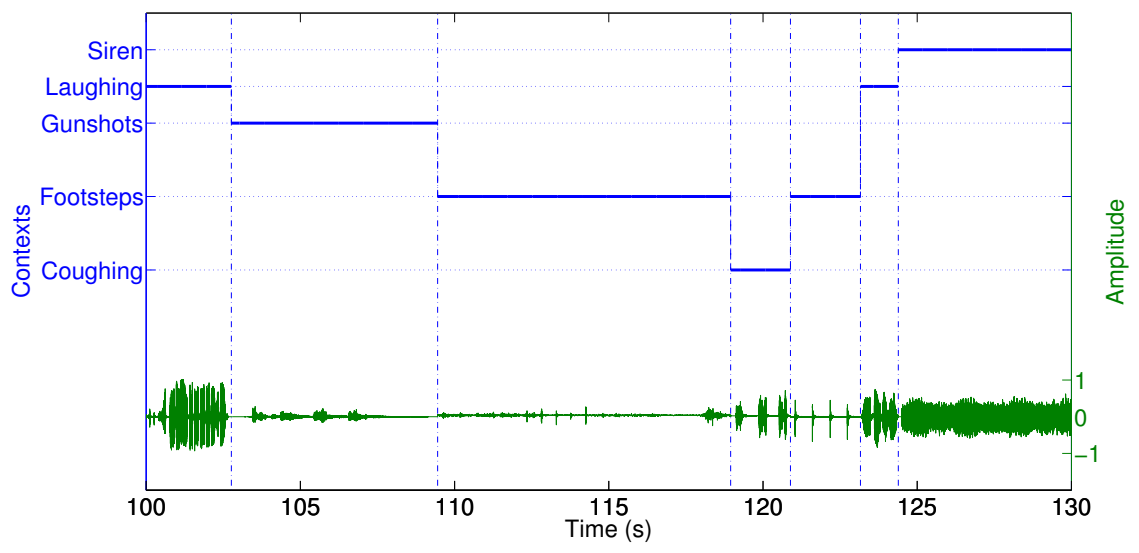

Figure 4.2: A random sequence of sounds.

based on change points can save the battery life by putting most power-hungry sensors in sleep mode whenever no change point is detected.

\subsubsection{Minimum Active Duration Sensing Scheduling (MASS)}

The MASS approach is proposed with a hypothesis that only sensory data sampled around the change points are interesting and informative enough to monitor the context of an event. Therefore, it requires only a minimum set of sensors, namely proactive sensors, to continuously monitoring context changes. Other sensors, namely reactive sensors, are set idle to save power consumption and will be invoked to enhance sensing data if a change is detected. In particular, whenever the sensing condition detected by Smartphone-Context Detector is matched, MASS activates only proactive sensors to monitor the events while reactive sensors are set idle. The incoming signals provided by proactive sensors are split into predefined overlapping time windows as illustrated in Fig. 4.3. Data features are extracted from the measurements within a window. An algorithm like an outlier detection algorithm would be used to detect if a change just happened.

Once a change point is detected, reactive sensors will be switched to their active modes to enriching data. Based on the likelihood of changing states, represented by a probability, MASS will define a sampling duration for reactive 


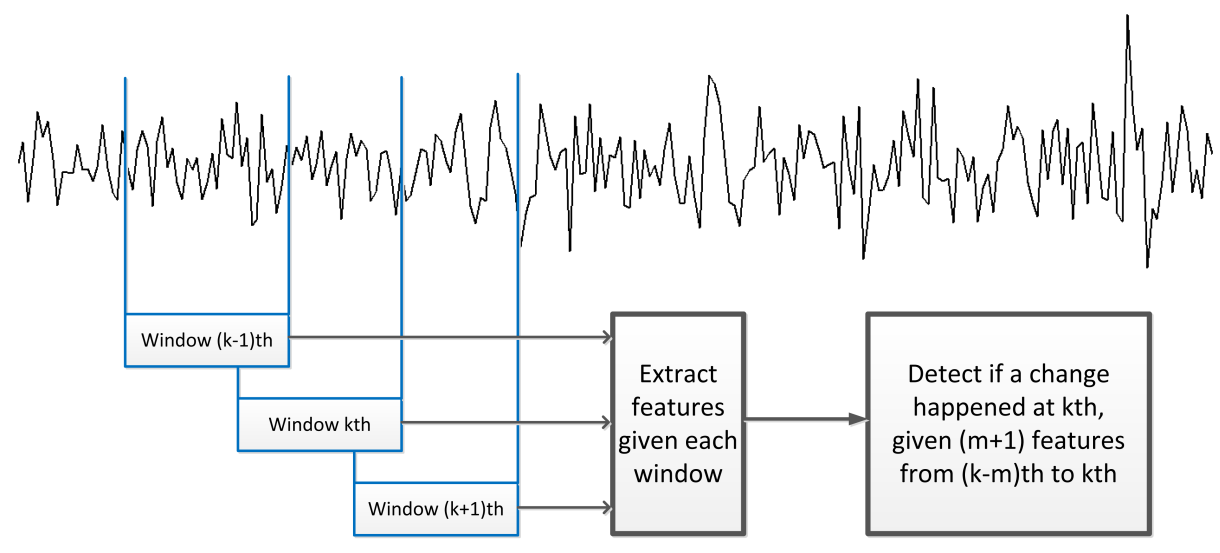

Figure 4.3: Online change detection framework.

sensors. The estimated sampling duration varies with specific applications. Nevertheless, the duration is required to be long enough so that the new states of the interesting event can be detected, for instant, it typically takes several seconds of audio samples to recognise sounds or human activities.

Note that we have presented MASS in the way that it can be applied for smartphones with different types of sensors, which are categorized into proactive and reactive groups. Nevertheless, MASS can be also applied for sensor devices that have only one sensor type. In this case, proactive sensors are a set of nodes that are active to monitor the context changes. Reactive sensors are sensors on the other nodes that are in idle mode and will be woken up once they received a trigger. The proactive set can be iteratively randomised or predefined, depending on applications and systems.

\subsubsection{Mathematical Formulation}

We consider a mobile sensing system with multiple mobile devices that are equipped with sensors and daily carried by users. The idea beyond our proposal is that the devices should be able to trigger sampling, processing and uploading sensory data around the change points in order to save sensing power and bandwidth. In other words, the proposed technique should be featherweight and able to quickly detect the change points on-fly with minimum false 
positive. To present the problem of online change detection more clearly, we first describe following definitions.

Definition 4.1. Let $\mathrm{T}$ denote an infinite set of discrete instants $t \in \mathrm{T}$ in time domain. Therefore, the timestamp value, which is assigned to each consecutive sample, can be regarded as natural numbers from $\mathbb{N}$. We define time intervals $\left(t_{1}, t_{2}\right]=$ $\left\{t \in \mathbb{T}, t_{1}<t \leq t_{2}\right\},\left[t_{1}, t_{2}\right)=\left\{t \in \mathbb{T}, t_{1} \leq t<t_{2}\right\}$ and $\left[t_{1}, t_{2}\right]=\{t \in$ $\left.\mathrm{T}, t_{1} \leq t \leq t_{2}\right\}$.

Definition 4.2. At an arbitrary $t$, a data stream $X$ is considered as an ordered sequence of the sample $x_{t}$, obtained with timestamp $t$. Without loss of generality, we define window $W_{t}$ is a set of temporal samples spanning $\omega$ units backwards from time $t, W_{t}=\left\{x_{i}, i \in(t-\omega, t]\right\}$, where $\omega$ denotes the window size.

Definition 4.3. To control the transition of successive windows $W$, we define sliding step of $\vartheta$ time units. Upon sliding, window $W_{t}^{k}$ at sliding step $k^{\text {th }}$ subject to $t=$ $k \vartheta+\omega, W_{t}^{k}=\left\{x_{i}, i \in(k \vartheta, k \vartheta+\omega]\right\}$ includes not only fresh $\vartheta$ samples but also a $\omega-\vartheta$ of samples from the previous window. For notational simplicity let $W^{k}=W_{t}^{k}$, the window at sliding step $k^{\text {th }}$.

Definition 4.4. For better representing information of data, we define data features or window features are the features that are extracted by mechanism $\mathcal{H}(\cdot)$. At any sliding step $k^{t h}$, a feature is computed by $F^{k}=\mathcal{H}\left(W^{k}\right)$ or $F^{k}=\mathcal{H}\left(x_{i}\right), i \in(k \vartheta, k \vartheta+$ $\omega]$. Note that $F^{k}$ is a feature vector with $p$ numerical attributes that represent data $W^{k}$. Therefore, feature $F^{k}$ can be also expressed as a row vector of $p$ elements, $F^{k}=$ $\left(a_{1}^{k}, a_{2}^{k}, \ldots, a_{p}^{k}\right)$.

Definition 4.5. Since the accumulative number of features $F$ in feature domain $\mathbb{R}^{p}$ can be very large and we only need to temporarily keep a finite number of historical features, we define the historical data at sliding step $k^{\text {th }}$ is a set of temporary features spanning $m$ units before $k^{t h}, \Gamma^{k}=\left\{F^{i}, i \in[k-m, k)\right\}$, where $m$ is namely historical 
length. For better visualisation, we express $\Gamma_{k}$ as a $m \times p$ matrix

$$
\Gamma^{k}=\left[\begin{array}{cccc}
a_{1}^{k-m} & a_{2}^{k-m} & \ldots & a_{p}^{k-m} \\
a_{1}^{k+1-m} & a_{2}^{k+1-m} & \ldots & a_{p}^{k+1-m} \\
\ldots & \ldots & \ldots & \ldots \\
a_{1}^{k-1} & a_{2}^{k-1} & \ldots & a_{p}^{k-1}
\end{array}\right]
$$

From above definitions, we have the mathematical formulation of the MASS problem and the change detection as the follows.

Definition 4.6. (MASS) given a streaming data $X$ sampled from consecutive context states that is composed of $n$ change points and have the length $\mathcal{T}$ time units, window size $\omega$, sliding step $\vartheta$ and current timestamp value $t_{c}$, MASS needs to seek an upcoming schedule to switch additional sensors into active mode whenever the context changes so that the total time in which the sensors are in active mode is minimized subject to that the sensing schedule covers the change points within an acceptable tolerance.

Definition 4.7. (Change Detection) Given $\Gamma^{k}=\left\{F^{i}, i \in[k-m, k)\right.$ is the training set and $F^{k}$ is the test sample, where $m \in \mathbb{N}$ is considered as the number of observations. By assuming that observed features belong to a single class, change detection detects if the current feature value $F^{k}$ belongs to another class. Mathematically, the problem is to find the statistical model probability $p(F)$. Then if

$$
\left\{\begin{array}{l}
p\left(F^{k}\right) \geq \epsilon, \quad \text { context is remained } \\
p\left(F^{k}\right)<\epsilon, \quad \text { context has changed }
\end{array}\right.
$$

where $\epsilon$ is a threshold, which can be chosen in advance or tuned by maximizing the F-measure in statistics.

In order to optimize MASS, the change detection needs to be able to:

- have lightweight computation.

- detect as many real change points as possible,

- detect changes quickly within a certain delay of milliseconds,

- keep as fewer false detections as possible. 


\subsection{Context Change Detection}

In this section, given sensory data, we introduce a new online change detection technique, namely Frequency Likelihood Estimation (FLE). The FLE algorithm provides a low false positive while maintaining a high true positive compared to current techniques. In other words, MASS implemented the FLE algorithm would enable researcher to develop energy-efficient applications including data sampling, data processing and data gathering with minimum resource consumption: computation, storage and battery.

\subsubsection{Histogram Likelihood Change Detection}

There are numerous algorithms for change detection, which are well reported in two surveys of Chandola et al. [66,67]. Among them, classification-based techniques are the most effective. Two most well-known techniques are the One-Class Support Vector Machine (OC-SVM) for outlier detection using Hamming distances [68] and the Multivariate Gaussian Distribution (MVN) in the Machine Learning course [69]. The OC-SVM is well known for its sensitivity and MVN is well known for its computational complexity. In particular, the OC-SVM can deal with a small number of samples but requires high computation. Conversely, the MVN is less effective but lightweight. In general, detecting change points from online streaming data given short historical samples (say from 10 to 50 samples) is still an intricate research topic for mobile sensing platforms. However, most classification-based techniques require adequate samples to train the parameters and the context is assumed to be remained after training. These requirements are not practical for mobile sensing, of which sensing context are dynamic and unpredictable.

In addition, we realize that most statistical change detections including the glshbos algorithm [70] are either too sensitive to outliers or too robust with statistical dispersion (underlying of statistical samples). This characteristic makes these change detections suitable for detecting outliers that are significantly different from a dispersion like the normal distribution, but not suitable for detecting changes that cannot be seen clearly such as from crying to laughing given audio data. To detect these sorts of changes efficiently, we propose the lightweight FLE algorithm, a nonparametric-based technique preliminarily described in [71]. The technique is termed "frequency likelihood" since we modify the conventional frequency histogram of consecutive samples. We reemphasize that using the histogram to detect anomalies has been used in previous work. However, histogram-based techniques have to endure the issues caused by the 
highly dynamic contexts. In particular, a small set of samples measured in a dynamic environment typically is not normal distributed, even left-skewed or right-skewed. Determining a fixed threshold also struggles with the dynamic. Note that the conventional Histogram-based Outlier Score (HBOS) constructs the bin width solely based on historical samples. However, the number of historical samples are quite short for online detection. Therefore, the HBOS has a poor performance since the test samples usually fall out of the predefined histogram. Indeed, our new method is able to deal with such issues.

Given temporary dataset $m+1$ samples including training set $\Gamma^{k}$ and test sample $F^{k}$, which is expressed as a $(m+1) \times p$ matrix

$$
\mathcal{D}=\left[\begin{array}{cccc}
a_{1}^{k-m} & a_{2}^{k-m} & \ldots & a_{p}^{k-m} \\
a_{1}^{k+1-m} & a_{2}^{k+1-m} & \ldots & a_{p}^{k+1-m} \\
\ldots & \ldots & \ldots & \ldots \\
a_{1}^{k} & a_{2}^{k} & \ldots & a_{p}^{k}
\end{array}\right]
$$

for each attribute column of $\mathcal{D}$, FLE first counts the amount of elements that fall into each disjoint category, also called bin. Let $b$ denote the total number of bins, FLE for each attribute $i, i=1, \ldots, p$, is a function $p_{i, j}$ that must satisfy:

$$
m+1=\sum_{j=1}^{b} p_{i, j} .
$$

In general, $b$ is set to $b<(m+1)$ to make use of the histogram. Unlike HBOS using only $m$ samples, FLE includes the test sample $F^{k}$ when building the histogram. This solves the case of that a test sample falls out of the histogram. It also turns out that the frequency $p_{i, j}$ at bin $j$ will have the highest value for attribute $i$ if there exists a change point. That the highest values of frequencies in the modified histogram are almost similar makes it feasible to chose a constant threshold.

Another issue is that the position of the maximum frequency is highly dynamic and depends on which bin most samples fall into. Consequently, repeatedly finding the location of the means consumes more mobile platform's resources. FLE can overcome this issue by taking the absolute values of given data as the data set $\mathcal{D}$. As a result, this technique most likely pushes the mean and outliers to the most left bin (called least significant bin LSB) and the most right bin (called most significant bin MSB). Therefore, we only need to simply count the frequencies of the LSB and MSB. The change probability of test data 
$\left|F_{i}^{k}\right|$ of the $i^{t h}$ attribute, denoted by $\widetilde{p}\left(\left|F_{i}^{k}\right|\right)$, can be estimated by:

$$
\widetilde{p}\left(\left|F_{i}^{k}\right|\right)=p_{i, 1}+p_{i, b}
$$

where $p_{i, 1}$ and $p_{i, b}$ are the frequencies of the LSB and MSB regarding to the $i^{\text {th }}$ attribute, respectively. Note that HBOS has to count frequencies of all bins, which requires more computation.

As our goal is to detect anomalies, we aggregate such individual change probability for all attributes of a feature by finding the maximum value. We remark that using joint probability will result in limiting the possibility to detect outliers since $\widetilde{p}\left(\left|F_{i}^{k}\right|\right)$ may have very small values. For instance, a buzzer emits considerable amplitude in high frequencies but not in low frequencies. Choosing the max probability is a better solution to increase the possibility of detecting the buzzer sound. Hence, the estimate of change probability given current feature observation $F^{k}$ is

$$
\widetilde{p}\left(\left|F^{k}\right|\right)=\max \left\{\widetilde{p}\left(\left|F_{i}^{k}\right|\right)\right\}, i=1, \ldots, p .
$$

To match (4.6) with the problem definition (4.2), we consider the density probability of the complement by computing the complement of the change probability.

$$
p\left(\left|F^{k}\right|\right) \leftarrow 1-\widetilde{p}\left(\left|F^{k}\right|\right) .
$$

Using above (4.7), we can detect changes using the condition described by (4.2).

\subsubsection{Complexity}

Given a temporary dataset $\mathcal{D}$ including training set $\Gamma^{k}$ and test sample $F^{k}$. The total number of entities in $\mathcal{D}$ is $p \times(m+1)$. For each attribute of feature $F_{i}$, FLE uses $m+1$ elements. Therefore, it requires $m+1$ operations to find the $\min$ and max values. It also needs another $m+1$ operations to count the frequency density of the LSB and MSB. Therefore, the complexity, or big-O of FLE is:

$$
O(2(m+1) p) \simeq O(m p),
$$

where $m$ is the history length and $p$ is the dimension of the data feature. We remark that the conventional histogram HBOS requires $b$, the number of bins, iterations to compute frequency density for each element. Therefore, the complexity of FLE is $b$ times less than that of the HBOS, which is $O(m b p)$. Moreover, the complexity of FLE is much less than that of the OC-SVM, $O\left(m^{3} p^{3}\right)$, and even the well-known lightweight MVN, $O\left(m^{2} p^{2}+p^{3}\right)$ 
With regards to the framework in Fig. 4.3, the total computational complexity, denoted by $C$, computed by summing feature extraction and detection technique costs. For example, the computational cost of HBOS with the MedianX feature extraction is

$$
C=O(q(m p+\omega)),
$$

where $q$ is the total quantity of sliding steps.

\subsubsection{Evaluation Metrics}

Since detecting changes from streaming data based on pattern recognition always has a delay of getting enough information, we leverage true positive (TP) and false negative (FP) [72] by adding latency parameter $\zeta$ in time domain. For better understanding, we interpret these definitions in context change detections. Assume that we expect there is no change of context, the null hypothesis $\left(H_{0}\right)$. Let $H_{a}$ denote the alternative hypothesis, there is a change happened within previous $\zeta \mathrm{ms}$. The error types then can be redefined as the follows.

- $T P_{\zeta}$ is the total number of real changes are detected within acceptable latency $\zeta$. The number of correctly detecting a change when $H_{a}$ is true.

- FP is the total number of wrongly alarms about a change. The number of falsely detecting a change when $H_{0}$ is true.

The value of $\zeta$ is set based on application requirements. However, it should not be smaller than the duration of the shortest context, for example, we set $\zeta$ to $1000 \mathrm{~ms}$ in our experiment. Based on these errors, we define the following metrics to evaluate the performance of change detection: Sensitivity $S$ and Efficiency $E$.

$$
S=100 \frac{T P_{\zeta}}{n}
$$

and

$$
E=100\left(1-\frac{T P_{\zeta}+F P}{q}\right),
$$

where $n$ is the number of real changes and $q$ is the number of total population or detection times.

We remark that sensitivity does not count the changes that are detected later than $1 \mathrm{~s}$. To evaluate how fast changes can be detected, we investigate the required latency $L_{S}$ to obtain the expected sensitivity $S$. Together with the aforementioned computational complexity $C$, these three metrics perfectly 


\begin{tabular}{|c|c|c|c|c|}
\hline Feature spaces & Notations & Description & $p$ & Complexity \\
\hline \multirow{3}{*}{ spectral } & PSDP & Power spectral density peak & 1 & $O\left(\omega \log _{2} \omega\right)$ \\
& SBC & Spectral subband centroids & 4 & $O\left(\omega \log _{2} \omega\right)$ \\
& SBCR & Spectral subband centroid ratio & 4 & $O\left(\omega \log _{2} \omega\right)$ \\
\hline cepstral & MFCC & Mel-frequency cepstral coefficients & 20 & $O\left(2 \omega \log _{2} \omega+\omega\right)$ \\
\hline principal & PCA & Principal component analysis & 20 & $O\left(\omega \log _{2} \omega+\omega^{2} / 4\right)$ \\
\hline temporal-spectral & DWT & Discrete wavelet transform & 4 & $O\left(\omega \log _{2} \omega\right)$ \\
\hline & ZCR & Zero-corssing rate & 1 & $O(\omega)$ \\
& MinX & Minimum amplitude & 1 & $O(\omega)$ \\
& MaxX & Maximum amplitude & 1 & $O(\omega)$ \\
& IqrX & Interquartile range & 1 & $O(\omega)$ \\
& MedianX & Median amplitude & 1 & $O(\omega)$ \\
& MeanX & Mean amplitude & 1 & $O(\omega)$ \\
& StdX & Standard variance amplitude & 1 & $O(\omega)$ \\
\hline
\end{tabular}

Table 4.1: Features used in the experiments.

represent the change detection problem defined in Section 4.4.3. A high value of sensitivity $S$ means a high number of real change points that are detected. A high value of efficiency $E$ means a low number of false detections. Note that execution time of algorithms does not really represent their computational costs since it heavily depends on the implementation optimization. Therefore, we prefer the computational complexity over the execution time.

\subsection{Experiments}

In this section, we first describe the experimental setup and then analyze the results with an audio dataset. The following experiments are aimed to evaluate (i) the FLE together with the OC-SVM and MVN and (ii) thirteen types of feature extractions shown in Table 4.1. These features are well-known for audio processing and classification [73]. In particular, we focus on the performance of such techniques when combining them with various feature types, especially light computational features. Note that we also conduct experiments with other types of sensor such as accelerometers, gyroscopes, magnetic sensors, barometers, and gravity sensors. The experiment results are published in [74].

\subsubsection{Experimental Setup}

Without loss of generality, we generated a database by randomly mixing 200 sound tracks of 10 common sounds (20 tracks per sound), which we may encounter in daily life: babies crying, bells ringing, cars honking, humans cough- 
ing, dogs barking, footsteps, glass breaking, gun shoots, human laughing and siren. Sound tracks are downloaded from the free database [75], and have various lengths from 1 second to 10 seconds. Through our experiment on Nexus 7, we experienced that real-time processing takes a considerable and unpredictable extra delay for each window, up to $500 \mathrm{~ms}$. The major cause comes from the non-deterministic audio latency, thread interrupts, and sound echoes. Such unknown latency results in inaccurately evaluating the real-time performance of change detection algorithms. To cancel the effects of such latency, we process the database offline as an online stream of audio with $20 \mathrm{~ms}$ windows and $10 \mathrm{~ms}$ sliding steps. We extract thirteen different features as described in Table 4.1, which cover the most common feature spaces. Note that the Blackman Harris window is used to reduce leakage within a Fourier Transform analysis.

We compared the change detection using the FLE against the HBOS, OCSVM, and MVN. The OC-SVM is one of the best anomaly detection technique in terms of sensitivity and the MVN is one of the best in terms of computational complexity, which are used most for anomaly detection. The algorithms are implemented and evaluated in Matlab given the dataset comprised from [75]. Consistent with our theoretical analysis, the HBOS performs poorly with the dataset. Therefore, we exclude the results of HBOS from our discussion. We set the bin length $b$ to 10 for the FLE, the radius of radial basis function kernel $\gamma$ to $1 / p$ for the OC-SVM and the significant level of $\mathrm{t}$-distribution $\alpha$ to 0.05 for the MVN as common setting values used in other work. The metrics described in Section 4.5 .3 are used to evaluate performance in terms of sensitivity, latency and efficiency. The detection algorithms were repeatedly run with different lengths of historical sliding steps, from 10 to 50 steps, or $100 \mathrm{~ms}$ to $500 \mathrm{~ms}$. More than $500 \mathrm{~ms}$ is not practical since the time between two consecutive change points can be $1 \mathrm{~s}$.

\subsubsection{Experiment Results}

Since the length of a sound track in our database can be as short as $1 \mathrm{~s}$, we only count detected changes with a delay less than $1 \mathrm{~s}$. In addition, the sensitivity $S$ is very critical in most applications. Therefore, the runs which have a sensitivity of below $95 \%$ will be ignored in our analysis.

Although we conducted the experiment with various features, we only show results of the features which are best presented for each feature space as listed in Table 4.1. The selected features are the Median Amplitude (MedianX), Zerocrossing Rate (ZCR), Power Spectral Density Peak (PSDP), Mel-frequency Cep- 


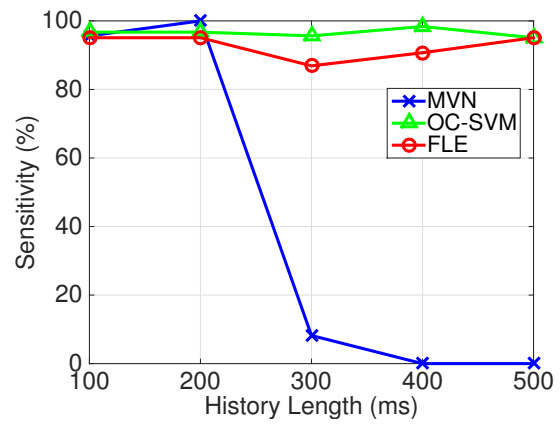

(a)

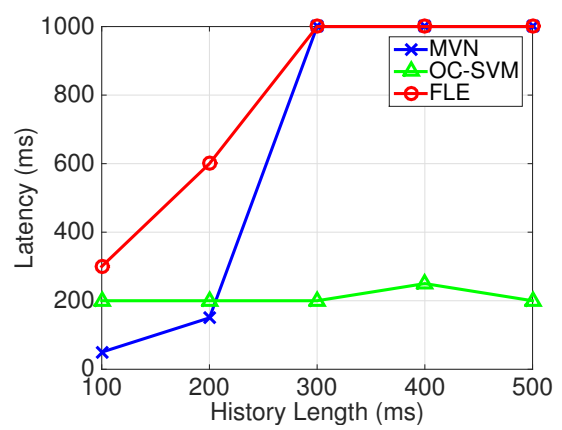

(c)

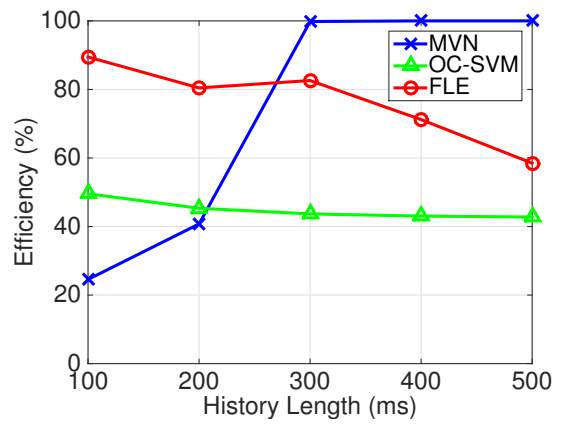

(b)

Figure 4.4: Change detection performance with the MedianX feature (a) Sensitivity, (b) Efficiency and (c) Latency.

stral Coefficients (MFCC), Principal Component Analysis (PCA), and Discrete Wavelet Transform (DWT), which are implemented in Matlab. Fig. 4.4 shows the performance of the change detection algorithms with the MedianX feature. By looking at both Fig. 4.4(a) and (b), we observe that FLE scores best in terms of efficiency as shown in Fig. 4.4(b), while still being able to detect real changes at high rate as we expected, see Fig. 4.4(a). Conversely, the MVN fails to detect the change points when the history length is over $200 \mathrm{~ms}$ as shown in Fig. 4.4(a). Therefore, the high efficiency of the MVN as shown in Fig. 4.4(b) is meaningless. In fact, the MVN is not able to detect changes when the history length is above $200 \mathrm{~ms}$. The OC-SVM performance scores best in terms of latency as shown in Figure 4.4(a). 


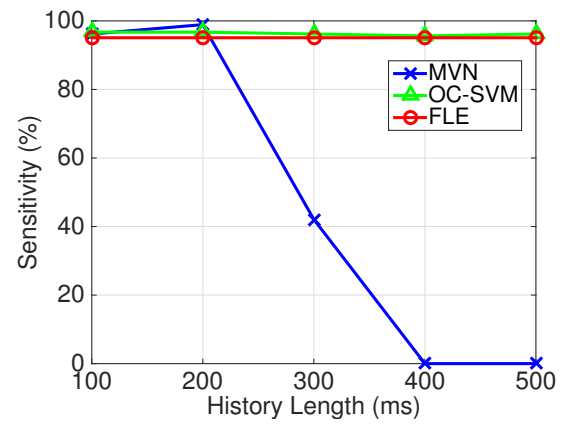

(a)

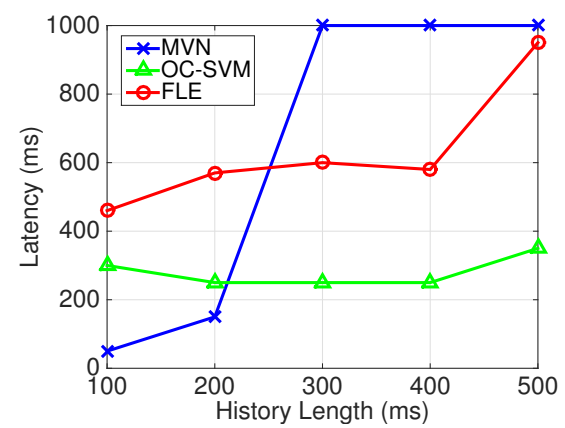

(c)

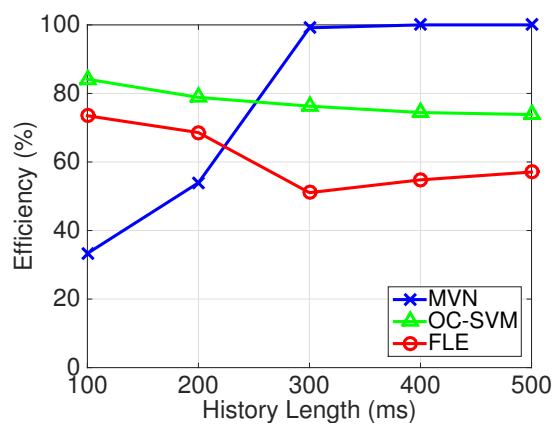

(b)

Figure 4.5: Change detection performance with the ZCR feature (a) Sensitivity, (b) Efficiency and (c) Latency.

Since the ZCR feature has been frequently used in sound analysis, we also consider the ZCR in our study besides the MeanX even both of them belong temporal space. The results are shown in Fig. 4.5. Efficiency of FLE is around $60 \%$, a bit lower than ZCR. However, the efficiency is still good enough for most sensing applications. The OC-SVM scores best when using the ZCR feature: high sensitivity, high efficiency, and short latency. This is due to the that the OC-SVM is in situ designed for classification and the ZCR is more informative than the MeanX, especially when applying on sounds. Contrary to FLE and the OC-SVM, the MVN with the ZCR feature performs as poor as with the MeanX feature.

Fig. 4.6 shows the performance metrics of change detection with the PSDP 


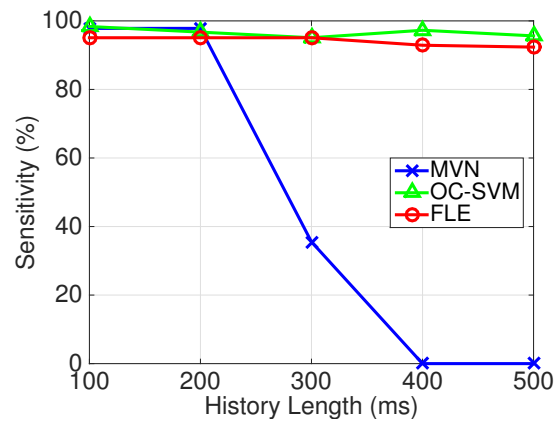

(a)

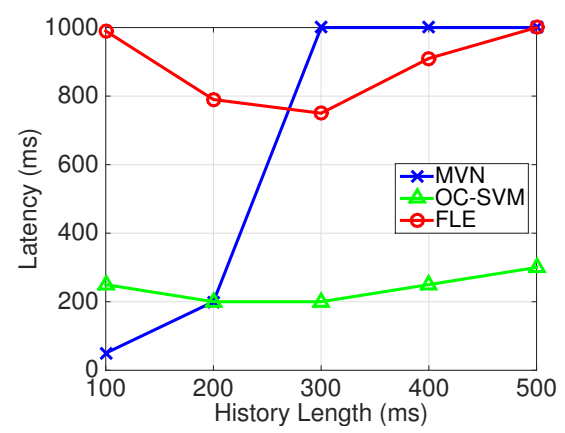

(c)

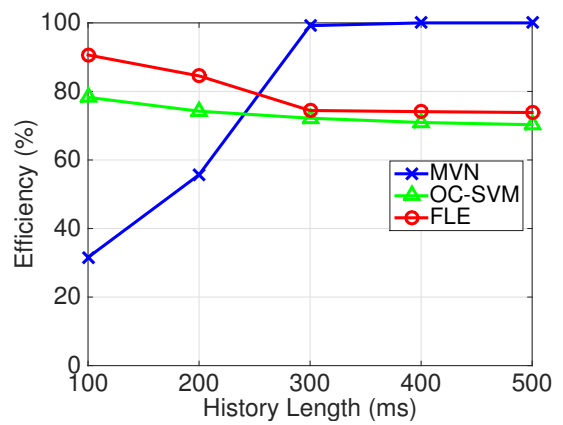

(b)

Figure 4.6: Change detection performance with the PSDP feature (a) Sensitivity, (b) Efficiency and (c) Latency.

feature when varying the history length parameter. Both FLE and the OC-SVM yield good results: high sensitivity ratios, high efficiency ratios and acceptable latency. FLE is better in terms of the efficiency while the OC-SVM is better in terms of the latency. Since the sensitivity ratios of MVN are very poor when the history length is greater than $200 \mathrm{~ms}$, its efficiency and latency results shown in Fig. 4.6(b) and Fig. 4.6(c) are meaningless.

By looking at the performance results with the MFCC shown in Fig. 4.7, we draw the same conclusions as with the PSDP. The similarity is due to that both PSDP and MFCC represent the frequency aspect.

Fig. 4.8 shows the results when applying changes detection with the PCA feature. And again, both FLE and OC-SVM perform well in terms of sensitivity, 


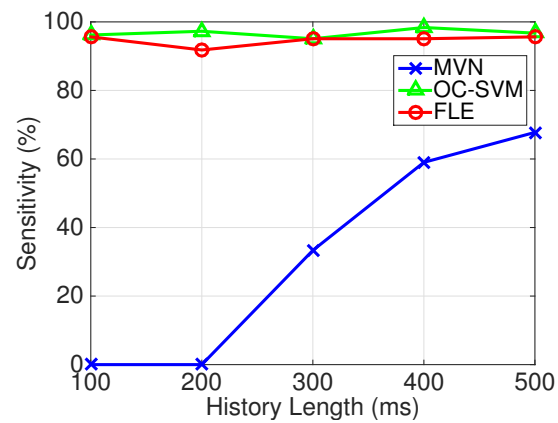

(a)

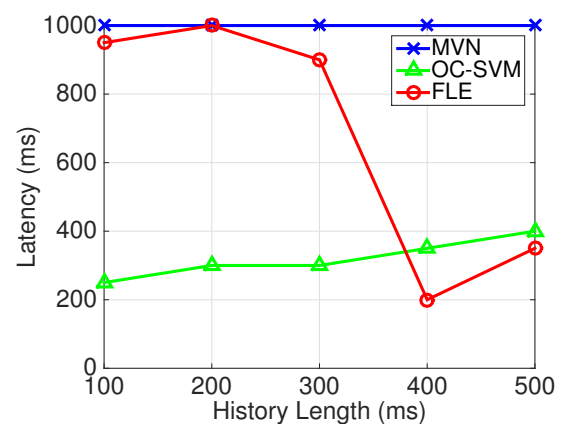

(c)

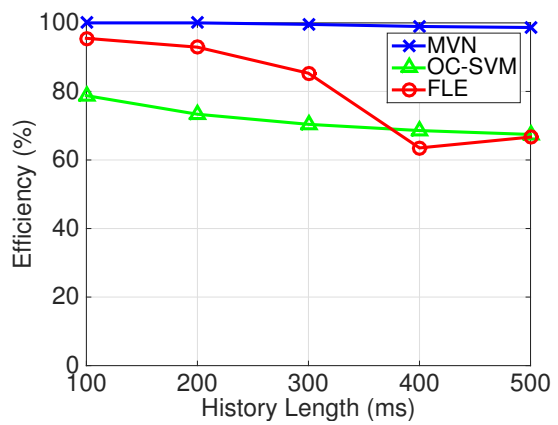

(b)

Figure 4.7: Change detection performance with the MFCC feature (a) Sensitivity, (b) Efficiency and (c) Latency.

efficiency and latency. Contrary to FLE and the OC-SVM, the MVN is not able to detect more than $95 \%$ of real changes. In addition, we observe that using the PCA gives shorter latency than using the MFCC. However, using the PCA gives lower efficiency, which means more false detections.

We have investigated change detection methods with features extracted in time and frequency domains. We will go on with another feature in timefrequency domain, the DWT feature. The experimental results are shown in Fig. 4.9. Surprisingly, FLE has a very low efficiency. In other words, FLE is not suitable for energy-efficiency sensing using the DWT. This is not what we expected but explainable. The fact is that DWT provides higher time resolution of high frequencies and lower time resolutions of lower frequencies. A small 


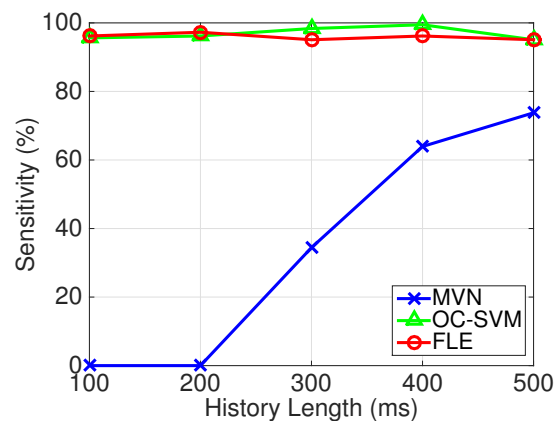

(a)

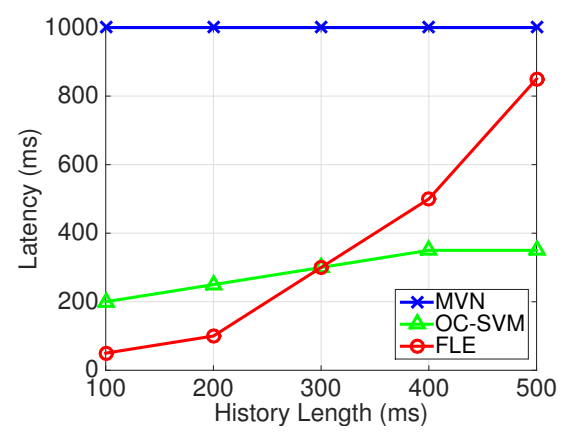

(c)

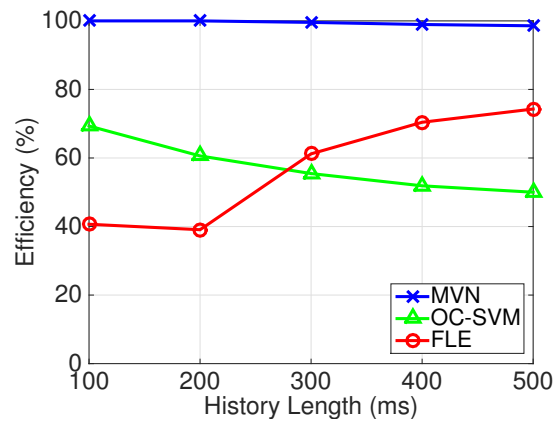

(b)

Figure 4.8: Change detection performance with the PCA feature (a) Sensitivity, (b) Efficiency and (c) Latency.

change in sensory data will result in a significant change in the historical pattern. This leads to very high sensitivity and short latency, but low efficiency. Due to the high resolution, the MVN is able to detect well real changes with acceptable efficiency.

Above conclusive results can be summarized as in Table 4.2 by averaging the performance results of history lengths. In the table, the hyphen "-" indicates the value is meaningless. The MVN is unsuitable for change detection in our assumption because it is not sensitive to the context changes. Overall, the OC-SVM performs best in terms of sensitivity and latency. The OC-SVM is able to detect more than $97 \%$ change points within approximate $300 \mathrm{~ms}$. We also observed that FLE is more efficiency than others, especially with the feath- 


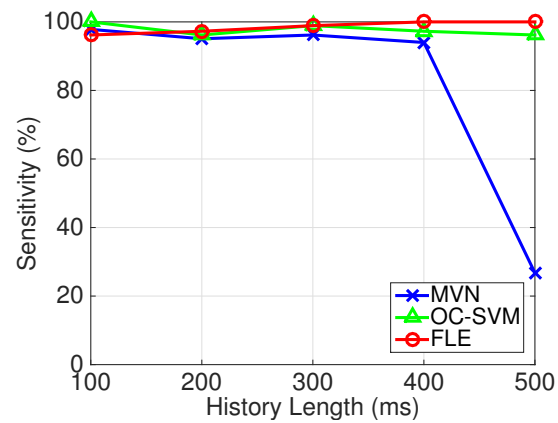

(a)

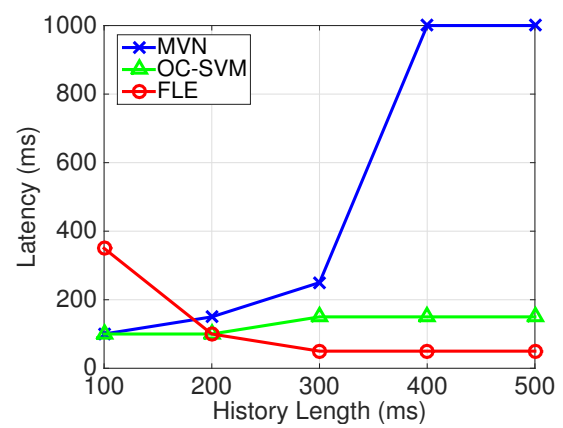

(c)

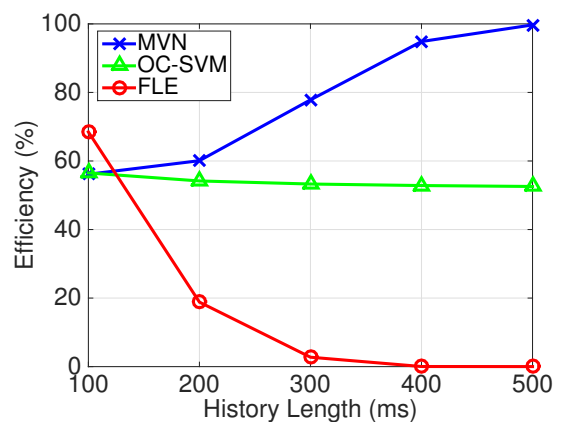

(b)

Figure 4.9: Change detection performance with the DWT feature (a) Sensitivity, (b) Efficiency and (c) Latency.

erweight feature MedianX. In addition, the computational cost of the combination of FLE and the MedianX is the least, see the numerical example calculated by (4.9) in Table 4.3. Since the objective of our problem is lightweight computing and high detection efficiency, FLE with the MedianX is a suitable solution for the energy-efficient problem. Moreover, FLE also performs well when using more complex features such as the PSDP and MFCC. In addition, the results clearly lead to a conclusion that the OC-SVM is suitable for applications of which high sensitivity and fast response are more important than resource saving. 


\begin{tabular}{|l||c|c|c|c|c|c|c|c|c|}
\hline \multirow{2}{*}{ Performance } & \multicolumn{3}{c|}{ MVN } & \multicolumn{3}{c|}{ OC-SVM } & \multicolumn{3}{c|}{ FLE } \\
\cline { 2 - 10 } & $\mathrm{S}$ & $\mathrm{E}$ & $L_{S}$ & $\mathrm{~S}$ & $\mathrm{E}$ & $L_{S}$ & $\mathrm{~S}$ & $\mathrm{E}$ & $L_{S}$ \\
\hline \hline MedianX & 41 & - & - & 97 & 45 & 210 & 93 & 76 & 780 \\
\hline ZCR & 47 & - & - & 96 & 78 & 280 & 95 & 61 & 540 \\
\hline PSDP & 46 & - & - & 97 & 73 & 240 & 94 & 80 & 780 \\
\hline MFCC & 32 & - & - & 97 & 72 & 320 & 95 & 81 & 680 \\
\hline PDA & 34 & - & - & 97 & 57 & 290 & 96 & 57 & 360 \\
\hline DWT & 82 & - & - & 98 & 54 & 130 & 98 & 18 & 120 \\
\hline
\end{tabular}

Table 4.2: Average performance values of history lengths.

\begin{tabular}{|c||c|c|c|c|c|c|}
\hline Big O & MedianX & ZCR & PSDP & MFCC & PDA & DWT \\
\hline \hline MVN & $4.55 \mathrm{E}+07$ & $4.55 \mathrm{E}+07$ & $1.28 \mathrm{E}+08$ & $1.38 \mathrm{E}+10$ & $1.42 \mathrm{E}+10$ & $6.19 \mathrm{E}+08$ \\
\hline OC-SVM & $6.62 \mathrm{E}+08$ & $6.62 \mathrm{E}+08$ & $7.44 \mathrm{E}+08$ & $5.19 \mathrm{E}+12$ & $5.19 \mathrm{E}+12$ & $4.16 \mathrm{E}+10$ \\
\hline FLE & $\mathbf{1 . 4 6 E + 0 7}$ & $\mathbf{1 . 4 6 E + 0 7}$ & $\mathbf{9 . 6 6 E + 0 7}$ & $\mathbf{2 . 3 5 E + 0 8}$ & $\mathbf{6 . 4 7 E + 0 8}$ & $\mathbf{1 . 0 2 E + 0 8}$ \\
\hline HBOS & $2.92 \mathrm{E}+07$ & $2.92 \mathrm{E}+07$ & $111 \mathrm{E}+08$ & $5.27 \mathrm{E}+08$ & $9.39 \mathrm{E}+08$ & $1.60 \mathrm{E}+08$ \\
\hline
\end{tabular}

Table 4.3: Numeric computation complexity of change detections with historical length is $200 \mathrm{~ms}$, given the experimental dataset comprised of 81105 sliding windows (160 samples per window).

\subsection{Summary}

In this chapter, we have proposed a cooperative distributed sampling method by optimizing the sampling schedule based on change detection. In particular, sensors are divided into two groups: the proactive and reactive group. Proactive sensors are supposed to actively monitor the interesting context, while reactive sensors are idle to save battery. Once the context changes, a trigger is generated to activate reactive sensors. The scheme, MASS is designed in a cooperative distributed manner. It also can be applied to multiple sensors types or single sensor type.

To enable MASS to be feasible, we proposed the nonparametric-based change detection FLE to detect change points of a context. The technique is featherweight, sensitive and efficient compared to existing ones such as the MVN, OC-SVM, and also HBOS. In particular, FLE estimates the density probability of a test sample using the frequency sum of the least and the most significant bins of the modified histogram. The complexity of FLE is only $O(m p)$, which is much lighter than the complexity of the MVN, OC-SVM and even HBOS.

Although the proposed method can be applied to various sensory data 
types, we conducted the experiment on sound data since the audio signal is likely more complex than others in terms of environmental noises, tempos, and dynamics. The experimental results are consistent with our analysis. The results show that FLE can detect more than $95 \%$ change points in limited time while saving $80 \%$ resource compared with standard continuous sensing. This work makes continuous sensing applicable for smartphone-based applications. In other words, it shows that using MASS with FLE can save significant amounts of sampling, processing and transmitting data. 


\section{CHAPTER 5}

\section{Non-deterministic Data Processing}

In the previous chapter, we studied techniques to efficiently sample sensory data with smartphones by intensively sensing data around the change points of interesting contexts. In this chapter, we present the solutions for the next layer of the architecture, the data processing stack. Although modern smartphones have provided a powerful platform to process sensory data locally, smartphones are non-deterministic. Therefore, sensory data collected by onboard sensors of smartphones are not as reliable as the data measured by dedicated sensors in the traditional Wireless Sensor Network (WSN). To this end, our goal is to explore and exploit opportunities given by smartphones to overcome the non-deterministic problem. In a cooperative distributed manner, we apply the non-deterministic algorithms to search for all possibilities to compute the outcome by varying the parameters then discarding the unreasonable solutions. The testbed results obtained through studying a distributed sound source localization provide a valuable insight into the proposed strategy, using non-deterministic algorithms to solve the non-deterministic problem of smartphones.

This chapter is partially based on:

- D.V. Le, J.W. Kamminga, H. Scholten, and P.J.M. Havinga "Nondeterministic Sound Source Localization with Smartphones in Crowdsensing," in Pervasive Computing and Communications Workshops (PERCOM Workshops), 2016 IEEE International Conference on. IEEE, 2016.

- D.V. Le, J.W. Kamminga, H. Scholten, and P.J.M. Havinga "Error Bounds of Localization with Noise Diversity," in Distributed Computing in Sensor Systems, 2016 IEEE International Conference on. IEEE, 2016. 


\subsection{Introduction}

The problem with using only the onboard sensors of smartphones without support from a dedicated sensing system has received comparatively little attention, especially with Android smartphone-based platforms that share more than $80 \%$ smartphone market in 2015. The main challenge is that Android devices have considerable uncertainties in time synchronization and signal processing latency. To investigate sensing latency, Perneel et. al. [76] conducted an experiment to measure the Android's real-time behavior and performance in terms of thread switch latency, interrupt latency, sustained interrupt frequency, mutex, and semaphore. The testing results showed that Android version 2.3.5 (Gingerbread) cannot be qualified to be used in real-time environments. Receiving numerous feedback from mobile developers on such latency, especially for audio, Google launched a set of guidelines to reduce the latency. However, the latency is still considerably large according to Google's measurements [77], even with the latest Android version such as Marshmallow 6.0 launched in October 2015.

In this chapter, we first study the behavior of data measurements with smartphone-based platforms. The results show that the measurement distribution is close to Gaussian only if there is a large dataset. Even though the distribution could be close to a normal distribution, the standard deviation is significantly large. Moreover, the distribution parameters vary with the device type. This means retrieving information using deterministic algorithms is a challenge because the number of poor measurements is comparable with good measurements, especially given a small sample size. Therefore, we propose to divide the measurements into subsets so that some subsets may contain mostly good measurements. Although insufficient samples provided by a single subset probably give inaccurate estimates, averaging such outcomes would deliver a higher estimation accuracy. This approach is a so-called non-deterministic algorithm, from which outcomes vary for every trial due to the impact of random parameters such as the subset size in our proposal.

As many pointed out, the non-determinism of Android operating systems in terms of audio latency is severe [77]. Meanwhile, audio is one of the most informative sources in mobile sensing and knowing a sound without its original location is meaningless in many sensing applications. Therefore, we applied the non-deterministic idea to a sound localization problem given only a set of smartphones and their acoustic Time Difference of Arrival (TDOA) measurements, without the help of extra infrastructures or dedicated sensing systems. In particular, we propose a Distributed variant of the RANdom SAmple 
Consensus (DRANSAC) scheme to deal with the effects of non-deterministic TDOA measurements on localization accuracy. Our testbed experiment results with 16 Nexus-7 tablets show that, when using the Levenberg-Marquardt (LM) method [78] under the DRANSAC scheme to solve the Least Absolute Deviation (LAD) cost function [79], the non-deterministic algorithm significantly improves the Root Mean Square Error (RMSE), two times better the deterministic version of the algorithm (1.19 $\mathrm{m}$ vs. $2.64 \mathrm{~m})$. An example is event detection within crowds, where people often carry their smartphones and there is no dedicated sound localization system.

In general, a Random Sample Consensus (RANSAC) scheme [80] can be used to weed out outliers in erroneously filtered estimated parameters, which are the unknown locations of the sounds in our problem. Such conventional RANSAC schemes require a pre-calibration to optimize RANSAC parameters, such as the number of iterations, the threshold of the number of inliers, and the threshold of the distances between the data points and the fitting line. Although the calibration is straightforward, it is ill-suited to dynamic environments. Therefore, we take advantage of the proliferation of smartphones to propose a distributed variant of RANSAC, as most people carry at least one smartphone wherever they go. This DRANSAC-like scheme distributes iterations to neighboring smartphones to perform decentralized optimization simultaneously using a deterministic fitting model. This approach eliminates the pre-calibration phase and allows the scheme to run faster since it uses a deterministic model to fit estimated outcomes rather than the RANSAC-based fitting. In addition, we propose to formulate the localization problem using the LAD cost function to lessen the influence of outliers in the TDOA measurements on the estimates.

The remainder of this chapter is organized as follows. Section 5.2 describes non-deterministic factors that effect measurements measured by smartphones. Our proposed DRANSAC scheme is presented through a sound localization in Section 5.3 and evaluated in Section 5.4. Finally, we conclude this work with Section 5.5.

\subsection{Non-determinism in Smartphone-based Sensing}

In this section, we discuss non-determinism in the smartphone-based platform through two aspects: the time synchronization among smartphones and the latency of signal transformation. Since smartphone-based platforms are nondeterministic, the synchronized clock and sensing latency contain considerable 
errors.

\subsubsection{Time synchronization}

When a Global Positioning System (GPS) radio is connected to multiple satellites the device can acquire very accurate time synchronization with the satellites. This accurate time $t_{g}$ is communicated through so called National Marine Electronics Association (NMEA) messages and has a resolution of nano seconds.

The NMEA messages are communicated to the Android framework from the GPS chip through several layers, which causes an offset between the timestamp stored inside the message and the moment the message received in the Android framework. This offset also varies and introduces jitter in the offset. The user application, that handles the received NMEA message in a callback, also suffers from latency. The user application handles the callback after a small delay, as Android is not a real-time OS.

The Android framework provides a timestamp $t_{s}$ along with the received NMEA message based on the system clock. This timestamp is the device's system time at the moment the Android framework received the message. The experimental application calculates the offset between the provided timestamp in the callback and the GPS-time, $\tau_{c}=t_{s}-t_{g}$. This offset is the parsed time from the NMEA message minus the timestamp that is provided in the callback.

In a very similar way, we found that clock synchronization using the Network Time Protocol (NTP) also faces an offset problem, the offset between the system clock and NTP server clock.

\subsubsection{Sensing latency}

Typically, a transducer takes a latency to convert a signal from a form of energy to another form of energy, usually electrical signal. The electrical signal also requires another delay to be converted from analog to digital. In addition, in multiprocessing platforms, the delay caused by interrupts and others concurrent tasks also affects the latency of measurements. To establish notations for future use, let $t_{r}$ denote the latency of measurements, which is from the moment measuring signal applied on the transducer till the moment its digital data are available in the input buffer for digital signal processing. Similarly, there is also latency denoted as $t_{e}$ from the moment the digital data is loaded into the output buffer to the moment the signal is converted into its physical form of energy, for example, an acoustic emitter sends out sound waves. 


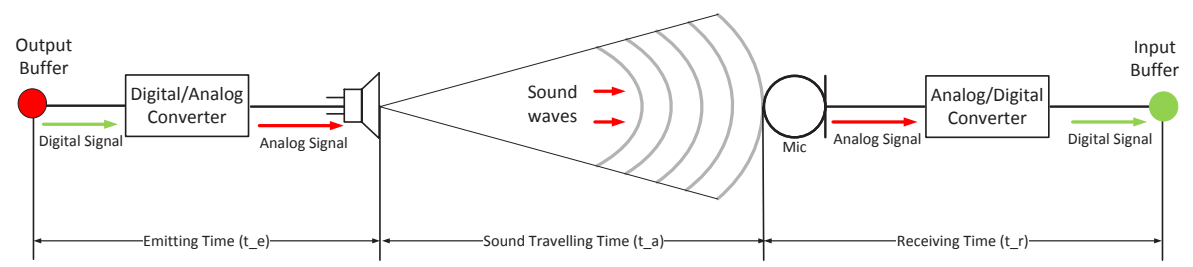

Figure 5.1: Sensing latency in measuring travel time of sounds. That converting time is usually unknown may result in unacceptable error in the measurements.

To make the problem clearer, we give an example on acoustic signals (sounds). Figure 5.1 illustrates the error in distance measurement based on acoustic sensors. For non-deterministic sensing platforms of ubiquitous devices, the delay from the moment when the sound reaches the microphone till the analog-todigital data are available at the input buffer can be in hundreds of milliseconds. In other words, the estimated distance from the sound source to the destination has an error of dozens of meters. This is unacceptable for most applications such as acoustic-based indoor localization. In analogy, emitting sounds via output buffer and speaker as illustrated in Figure 5.1 are equally erroneous.

As we assumed that we only can get timestamps when the data are loaded into the output buffer and when the data are available in the input buffer, the latency we can measure, denoted as $T$, includes the emitting latency $t_{e}$, the signal travelling latency $t_{a}$ and the receiving latency $t_{r}$.

$$
T=t_{e}+t_{a}+t_{r}
$$

Since our objective is to study the latency caused by signal conversion, we minimise the distance between a transmitter and a receiver, the signal travelling latency $t_{a}$ can be neglected in Equation 6.2. Let $\xi$ denote the sensing latency, we have $\xi \approx t_{e}+t_{r}$. The problem turns out how to measure and estimate the latency $\xi$.

\subsubsection{Experimental Evaluation}

From the best of our knowledge, the Android platforms have a poor reputation on time synchronization as well as audio sensing latency, but no up-to-date literature has studied the influence scientifically as far as we know. Therefore, 


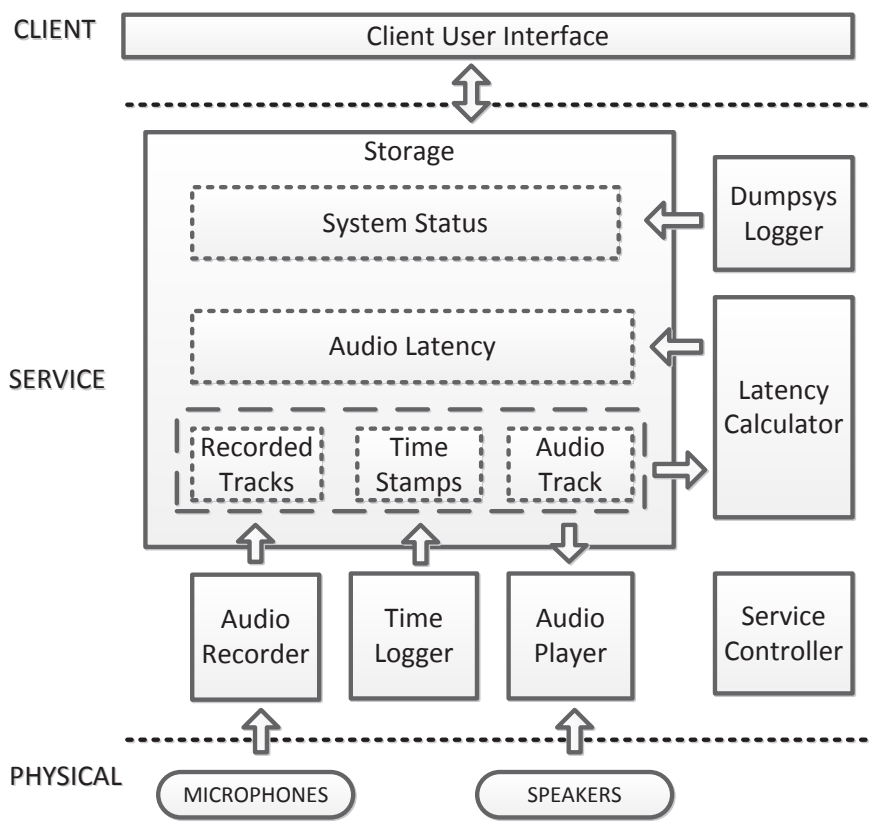

Figure 5.2: Architecture of the Acoustic Latency app.

we built an application to measure the errors of the time sychoronization and sensing latency. Software architecture of the app is shown in Figure 5.2. The architecture comprises seven software components: Service Controller, Audio Recorder, Audio Player, Time Logger, Dumpsys Logger, Latency Calculator, Storage and Client User Interface (UI). To ensure performance reliability, we implemented all components, except Client GUI, under a bound service app that runs in an independent process. Client UI is implemented as a client app that can bind to the service through interprocess communication. Moreover, audio recording and playing are implemented in NDK level to avoid delay due to the Java running environment.

To evaluate the theoretical analysis, we conducted a testbed experiment with audio signals. First we use the app to measure the clock system offset with regard to the clock from GPS chips of 16 Nexus 7 tablets, with Android 4.3 


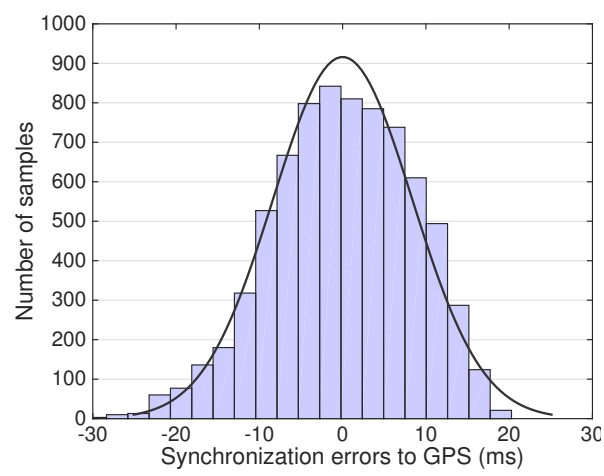

(a) Mean $=0 \mathrm{~ms}$ Std. $=8.3 \mathrm{~ms}$

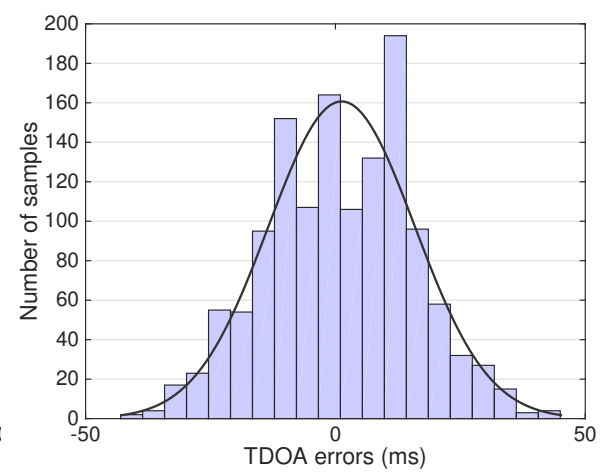

(b) Mean $=1.2 \mathrm{~ms}$

Std $=14.6 \mathrm{~ms}$

Figure 5.3: Testbed experiment: (a) GPS-based time synchronization, (b) TDOA measurements.

installed. The devices were placed outdoor without obstruction such as buildings and trees nearby to ensure good line of sight to satellites. Figure 5.3(a) shows the histogram with a Gausian distribution fits to the mean and variance of $15 \times 500=7500$ offset samples. The mean value is $0 \mathrm{~ms}$ and the standard deviation is $8.3 \mathrm{~ms}$. In other words, the error of acoustic ranging caused by inaccurately synchronized clocks of the devices in general can be modelled as a Gaussian distribution with zero mean and standard deviation is $2.8 \mathrm{~m}$, speed of sound is $0.34029 \mathrm{~m} / \mathrm{ms}$.

Next, we measure the variance caused by audio sensing latency. The delay is counted from the moment an acoustic signal arrives at the microphones to the moment the digital data of the signal is available to be processed in the reader buffer. There are a number of works have investigated such acoustic latency [81-83]. Reasons include interrupt inversion, pre-emption disability application priority, buffer size, signal processing, scheduling latency, power management, security kernels, etc. In our experiment, we placed aforementioned 16 Nexus 7 devices at a same position. Then we sounded a horn right above the tablets. Figure 5.3(b) shows the histogram and Gaussian distribution that fits with the mean and standard deviation of 1350 TDOA measurements. 


\subsection{Non-deterministic Algorithms for Smartphone- based Sensing}

Based on our study, we observed that most errors in measurements with smartphones are due to unpredictable outliers caused by jitter from the smartphone operating system. It is hard to remove these outliers since the quantity of outliers is comparable to the quantity of inliers, and the distribution knowledge of the measurements are unknown in most situations, for instance, the population mean and standard deviation. Therefore, most estimators have a poor performance given such non-deterministic measurements. In this section, we propose a new perspective on the non-deterministic problem via nondeterministic algorithms.

By definition, a non-deterministic algorithm is, as opposed to a deterministic algorithm, an algorithm that can produce different results with different runs for the same given data. The different behaviors on different runs can be due to a race condition, a random number generator, an initial value, etc. The non-deterministic algorithms are often used to find an approximate solution when the exact solution would be too costly or almost impossible to obtain using a deterministic one.

\subsubsection{Sound Source Localization Problem}

We present how to solve the non-deterministic problems caused by smartphonebased platforms through the sound source localization problem. The motivation is that time synchronization and audio latency are significantly large and non-deterministic with smartphones, especially the Android ones. However, sound source localization requires quite accurate measurements. The problem can be formulated as follows.

In general, we consider a network comprising a set $\boldsymbol{S}=\{\boldsymbol{x}(k): k=$ $1, \ldots, n\}$ of $n$ acoustic emitters with unknown location information, namely source nodes, and a set $\boldsymbol{A}=\{\boldsymbol{x}(k): k=n+1, \ldots, n+m\}$ of $m$ acoustic sensor devices such as smartphones with known location information, namely anchor nodes. To idealize the localization problem, we assume that all source nodes and anchor nodes are stationary on a 2-dimensional plane for a few seconds which is long enough to perform the distributed localization with smartphones. Localization with mobility and in a higher dimension is straightforward. We also assume that the size of each node is small enough to be treated as a point on the localization map, with has the coordinate $x=(x, y)^{T} \in \mathbb{R}^{2}$. 
Each source node generates a limited-power acoustic signal that can be used to estimate the distance to near receivers. Let $\boldsymbol{N}(k)$ denote the set of anchor nodes in the range of the $k^{t h}$ node. Clearly, $\boldsymbol{x}_{k} \notin \boldsymbol{N}(k)$ and $\boldsymbol{N}(k) \subset \boldsymbol{A}$.

The localization problem is to estimate the vector positions of the source nodes, the unknown parameter $\Theta=\left\{\theta(k)=\left(\hat{x}_{k}, \hat{y}_{k}\right)^{T}, k=1, \ldots, n\right\}$, given the vector positions of the anchor nodes in $\boldsymbol{N}(k)$ and the TDOA measurements $\left\{\delta_{i, j}(k): i, j \in \boldsymbol{N}(k), j \neq i\right\}$. In a 2-dimensional plane we have $\delta_{i, j}(k)=$ $c\left(t_{j}(k)-t_{i}(k)\right)$, where $c$ is the speed of sound $(\approx 0.34029 \mathrm{~m} / \mathrm{ms}), t_{i}(k)$ and $t_{j}(k)$ are the TDOA values of the sound source $k$ when the signal arrives anchor nodes $i$ and $j$, respectively.

Without loss of generality, we assume that all measurements $\left\{\delta_{i, j}(k): i, j \in\right.$ $\boldsymbol{N}(k), j \neq i\}$ between a blind node $\boldsymbol{x}(k)$ and its neighbouring anchor nodes in $\boldsymbol{N}(k)$ are available. We also assume that the distribution of $\delta_{i, j}(k)$ is Gaussian. In addition, we assume that $\left\{\delta_{i, j}(k)\right\}$ are statistically independent. This assumption somewhat oversimplifies the practical environment but it is necessary for analysis. Finally, we assume that there is an adequate number of anchor nodes. For example, at least three anchor nodes are required in any set of $\boldsymbol{N}(k)$ in 2-dimensional space.

In a real-world application, a source node can be either natural or synthesized. Some kinds of source nodes might have microphones to receive the acoustic signal as well, for example, smartphones that emit sounds. Therefore, $\boldsymbol{N}(k)$ might consist of not only anchor nodes but also source nodes that can receive the acoustic signal from the $k$ th node. Since most of the modern devices possess accurate positioning systems such as the acoustic localization system proposed by Liu et al. [84] could achieve an accuracy of $23 \mathrm{~cm}$, and our aim is sound source localization in crowds, we will focus on localizing natural sound sources.

\subsubsection{Time-of-Different Arrival Localization}

As the time of emission, also called Time of Arrival (TOA), is almost impossible to obtain for natural sounds, we propose to solve the sound localization problem based on TDOA. Herein we first mathematically model the TDOA measurements. We then introduce the mathematical problem of the TDOA localization. 


\section{Time Difference of Arrival Variables}

TDOA is used in the case where the time of sound emission is unavailable. The TDOA measurements can be obtained by computing the difference between the TOA of source node $k$ and the anchor nodes as

$$
\boldsymbol{\delta}(k)=\left(\begin{array}{c}
\delta_{2,1}(k) \\
\delta_{3,1}(k) \\
\vdots \\
\delta_{|\boldsymbol{N}(k)|, 1}(k)
\end{array}\right),
$$

where $\delta_{i, j}(k)$ is the TDOA measurement defined in Section 5.3.1. Since the TDOA measurement $\delta_{i, j}(k)$ is computed by multiplying the time-of-flight and the speed of sound, $\boldsymbol{\delta}(k)$ is a vector of Euclidean distances, which has the length depending on how many anchor nodes in the range node $k$. Note that the TDOA vectors $\{\boldsymbol{\delta}(k)\}$ may have different sizes.

\section{Least Squares Problem}

Typically, a localization problem can be formulated as a least-squares problem so that it can be optimized by using a least-squares optimization, such as the LM method [78] or Trust-Region-Reflective Algorithm [85]. Let $J(\boldsymbol{x}(k))$ denote the Jacobian (cost) function of the location estimation for source node $k$, we have

$$
J(\boldsymbol{x}(k))=\sum_{i=2}^{|\boldsymbol{N}(k)|}\left(\|\boldsymbol{x}(k)-\boldsymbol{x}(i)\|-\|\boldsymbol{x}(k)-\boldsymbol{x}(1)\|-\delta_{i, j}(k)\right)^{2}
$$

where $\|\cdot\|$ is the Euclidean norm and $|\boldsymbol{N}(k)|$ is the size of $\boldsymbol{N}(k)$. Recall that $|\boldsymbol{N}(k)| \geq 3$.

\section{Least Absolute Deviations}

As the Ordinary Least Squares (OLS) problem formulation amplifies the outliers of measurement by squaring the residuals, we propose to use the LAD instead. The Jacobian function then becomes

$$
J(\boldsymbol{x}(k))=\sum_{i=2}^{|\boldsymbol{N}(k)|}\left|\|\boldsymbol{x}(k)-\boldsymbol{x}(i)\|-\|\boldsymbol{x}(k)-\boldsymbol{x}(1)\|-\delta_{i, j}(k)\right|,
$$


Table 5.1: Comparing statistical optimality criteria

\begin{tabular}{|c|c|c|}
\hline Criteria & Least Squares & Least Absolute Deviations \\
\hline Robust & $\boldsymbol{x}$ & $\boldsymbol{X}$ \\
\hline Stable & $\checkmark$ & $\boldsymbol{\checkmark}$ \\
\hline Multiple outcomes & $\boldsymbol{x}$ & $\boldsymbol{}$ \\
\hline
\end{tabular}

where $|$.$| is the absolute value.$

Table 5.1 may help to understand why we choose LAD as the criterion for our localization optimization. Compared to the OLS method, the LAD method is more robust because it is resistant to outliers in the data, which are very common when measuring data with non-deterministic smartphone-based platforms. In addition, the instability property of the LAD method makes it self-adaptive to the change of the random parameter of non-deterministic approaches, for instance, the random subset of anchors is used to iteratively estimate the positions of source nodes. A small change in the configuration of input data may produce a significant difference in the optimization results. Finally, the LAD is also a better choice since it can provide multiple outcomes that offer an opportunity to select the best solution.

\subsubsection{Non-deterministic Localization Approach}

Since it would be too costly to obtain high localization accuracy by an exact solution, we propose to use nondeterministic approaches to find an approximation to a solution. In general, a nondeterministic algorithm is an algorithm that may provide different results on different runs, even for the same given input. To solve the sound source localization problem, we address a probabilistic algorithm of which behaviors rely on a random number generator. The random number can be the number of devices selected to perform distributed localization. Every estimated location the nondeterministic algorithm computes is valid, regardless of which set of devices the algorithm chose while running.

In particular, we propose to optimize the LAD problem using an iterative algorithm such as the LM method on random subsets. The bottom line of the idea is to minimize the effect of measurement outliers caused by the nondeterministic behaviors. The run of the algorithm with a random subset that contains mostly anomalies will result in a large residual, which can be filtered 
out later by some outlier detection, such as Online Frequency Likelihood Estimation $[74,86]$.

The localization algorithm is designed in a cooperative distributed manner. In other words, for each source node $k$, the localization computation tasks are split and assigned to different anchor nodes, which are smartphones in $\boldsymbol{N}(k)$. Then such anchors nodes combine their estimated models to enhance the estimated locations of the source node. More details of the algorithm are presented in Algorithm 5.1. Without loss of generality, the algorithm is expressed with regard to anchor node $i$ and source node $k$ for implementation clarity.

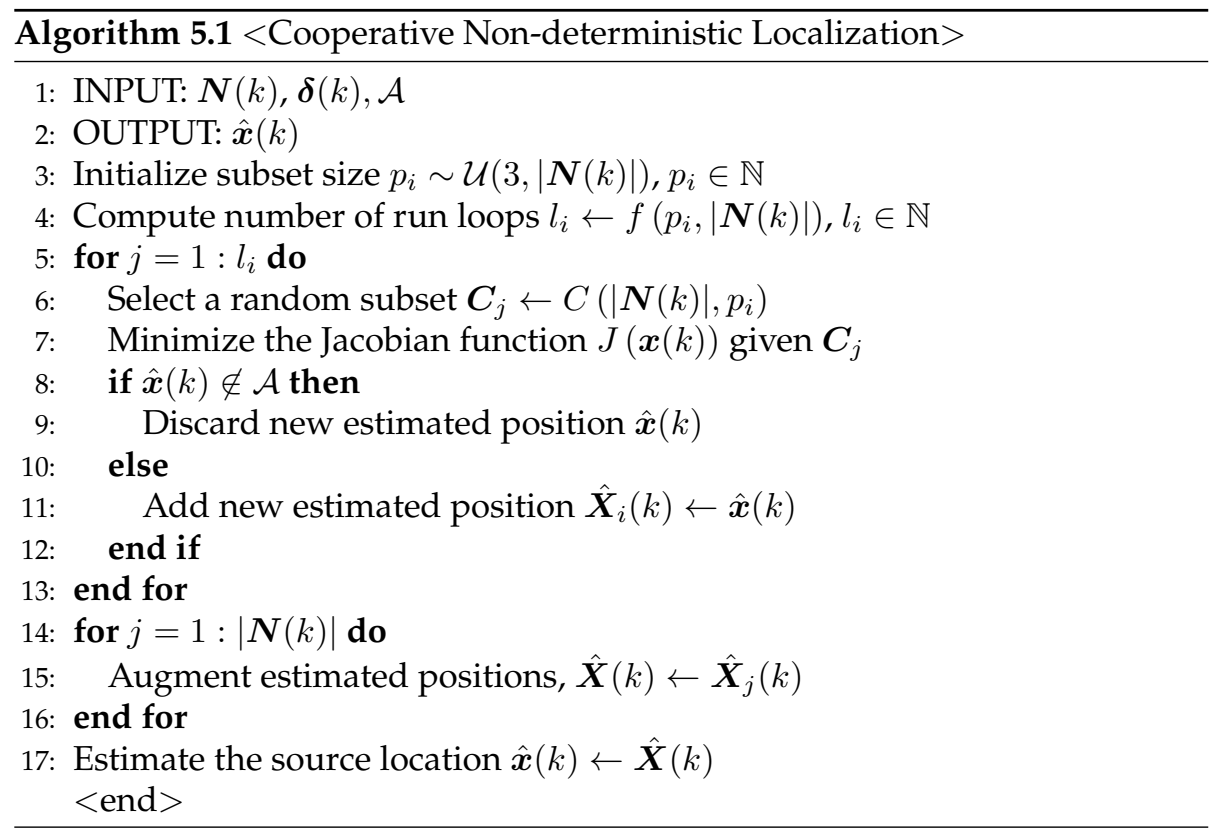

To run Algorithm 5.1 on an arbitrary anchor node $i$, it requires that smartphone $i$ has to connect to other smartphones in neighborhood $\boldsymbol{N}(k)$ to obtain the TDOA measurements $\boldsymbol{\delta}(k)$, which are correlated to a same sound source $k$, and estimate the area $\mathcal{A}$ bounded by anchors in $\boldsymbol{N}(k)$. The random size of the subset $p_{i}$, which will be used to localize source node $k$, is drawn from a discrete uniform distribution with lower and upper endpoints specified by 3 and $|\boldsymbol{N}(k)|$, respectively. Note that the minimum number of anchor nodes to perform $2 D$ localization is 3 . Line 3 in Algorithm 5.1 determines the number 
of loops $l_{i}$ to perform location estimation, $l_{i}=f\left(p_{i},|\boldsymbol{N}(k)|\right)$ given by (5.7). For each loop, a random subset $C_{j}$ is selected without replacement from all possible combinations of anchor nodes in $\boldsymbol{N}(k)$ taken $p_{i}$ at a time. Without replacement means that if a subset is already selected, it will not be selected again in the next loop. Given the TDOA measurements $\boldsymbol{\delta}(k)$ and the anchor nodes in $\boldsymbol{C}_{j}(k)$, the coordinates of source node $k$ are estimated by applying an estimator such as the LM on the non-linear cost function $J(\boldsymbol{x}(k))$ described in (5.3) or (5.4). If an estimated position $\hat{\boldsymbol{x}}(k)$ does not fall in area $\mathcal{A}$, it will be discarded. Note that other approaches use a RANSAC-based outlier detection instead of the area-based constraints like us. Combining the valid estimated coordinates of source node $k$ provided by other smartphones in the group, we obtain set $\hat{\boldsymbol{X}}(k)$ of which elements are considered as samples of a distribution. Finally, the expected value of the coordinate distribution is computed through lines 12 and 13, which is also the final estimated location of source node $|\boldsymbol{N}(k)|$.

In particular, the number of loops $l_{i}$ is computed based on the subset size $p_{i}$ and the neighbor-set size. A small subset will result in a higher variation of the estimated locations, and thus needs more loops to determine the inliers given by:

$$
l_{i}=\left\lfloor\beta \frac{|N(k)|}{p_{i}}\right\rceil,
$$

where $\lfloor$.$] is the nearest integer and \beta$ is the loop factor. The loop factor is the minimum number of loops that are required for a single anchor node (smartphone) to run all possible subsets of the smallest combination, which is when the largest possible subset size $p_{i}=|\boldsymbol{N}(k)|-1$. We have,

$$
\begin{aligned}
\min _{p_{i}} C_{|\boldsymbol{N}(k)|}^{p_{i}} & =C_{|\boldsymbol{N}(k)|-1}^{|\boldsymbol{N}(k)|-1}=\frac{|\boldsymbol{N}(k)| !}{(|\boldsymbol{N}(k)|-1) ! 1 !} \\
& =|\boldsymbol{N}(k)| \forall p_{i} \in[3,|\boldsymbol{N}(k)|] .
\end{aligned}
$$

Therefore, the number of loops can be rewritten as follows:

$$
l_{i}=\left\lfloor\frac{|\boldsymbol{N}(k)|(|\boldsymbol{N}(k)|-1)}{p_{i}}\right\rceil .
$$

Equation 5.7 tells that in the case of smallest combination, $p_{i}=|\boldsymbol{N}(k)|-1$, a single anchor node needs to run all the possible subsets, which is $l_{i}=|\boldsymbol{N}(k)|$. 


\subsection{Testbed Experiment}

In this section, we describe the testbed that is used to evaluate our proposed cooperative non-deterministic approach for sound source localization. We demonstrate our scheme with one of the best optimization methods, the LM method [78]. The LM method is used to solve non-linear least squares problems by interpolating between the Gauss-Newton algorithm and the method of gradient descent. If an iteration gives an insufficient reduction in the residual, the damping factor can be increased to bring the LM closer to the gradient descent, whereas if an iteration reduces the residual enormously, the damping factor can be decreased to bring the LM closer to the Gauss-Newton algorithm. The results show that non-deterministic approaches provide localization accuracy that is significantly better than the standard variants and very close to the Cramer-Rao Bound (CRB), especially when there are many smartphones participating in localization. The CRB is a benchmark that has been popular to evaluate localization algorithms [87]. It provides a lower bound of estimators given measurements containing Gaussian noises. The CRB can be computed from an inverse Fisher Information Matrix (FIM) in information theory given the Gaussian parameters of the noises.

\subsubsection{Experimental Setup}

In order to conduct the experiment, we developed a localization application and installed it on 16 Nexus-7 tablets. In particular, there are 16 Nexus-7 tablets installed with Android 4.4.4 (KitKat) marked as " $\square$ " that were deployed over an outdoor area with a pairwise distance of $4 \mathrm{~m}$ as illustrated in Fig. 5.4(a). From our experience in working with smartphone sensor networks, this experiment setup is very similar to daily circumstances such as in a classroom, in a conference, and on a walking-street. Since we address the environmental sounds within a crowd, sound sources were sequently generated at 9 positions marked as "o" in Fig. 5.4(a), five successive sounds per each position. The aim of conducting multiple measurements is to increase the possibility of position estimates. Although the positions of the tablets are known and fixed in our testbed, we placed them next to the absolute reference points with a random error of $\pm 30 \mathrm{~cm}$. This random placement intends to emulate the random errors of the device positions obtained by an off-the-shelf system such as the acoustic localization system proposed by Liu et al. [84]. 


\subsubsection{Experimental Results}

Fig. 5.4(b) shows sound sources and estimated locations for DRANSAC-LADLM using Nexus-7 tablets, which are marked as "o" and " $\triangle$ ", respectively. The dash eclipses illustrate the bounds of possible outcomes that vary around the mean of estimated locations. The non-deterministic algorithm has a RMSE of $1.19 \mathrm{~m}$, which is amazingly good since the standard deviation of TDOA measurements is approximate $5 \mathrm{~m}$. Especially, the localization error that can be achieved is very good at some locations such as an error of $28 \mathrm{~cm}$ for node number 2 . In consistency with our theoretical analysis, DRANSAC-like localization scheme efficiently deals with outliers of measurements when combining LAD and LM. In particular, our algorithm iteratively filters out outliers by assuming that there exist subsets of inliers from measurements. These inliers can be used to estimate the location of sounds. Sound node number 9 has the worst error since it produces a lot of outliers in our measurement data. Conducting more measurements would help to improve the accuracy.

On the other hand, Fig. 5.4(c) shows sound sources and their mean-estimates of sound locations with the deterministic approach LAD-LM using 16 Nexus7 tablets, which are marked as "o" and " $\nabla$ ", respectively. Consistently with the theoretical analysis, the deterministic algorithm mostly returns a constant estimated location for every source, of which RMSE is $2.64 \mathrm{~m}$. Note that both DRANSAC-LAD-LM and LAD-LM use the same measurement inputs, of which outliers are removed based on the mean and standard deviation of the TDOA measurements.

That is to say DRANSAC-LAD-LM can remove further outliers from the TDOA measurements through estimates whereas LAD-LM cannot. As a set of pairwise measurements may contain largely outliers due to a channel shadowing, an outlier detection algorithm will remove inliers, but outliers. This explains why LAD-LM still has poor accuracy even if applying outlier detection on the inputs. However, the effects of outliers can be revealed after using them to estimate the unknown location. The estimated location would be unrealistic and easier to be filtered with a constraint, for example, with the area formed by the locations of anchor nodes.

It is interesting to study the computational load of non-deterministic localization through how fast a non-deterministic localization converges to a stable accuracy. Fig. 5.5(a) shows the convergence speeds of LAD-LM (deterministic localization algorithm) and DRANSAC-LAD-LM (non-deterministic localization algorithm). Surprisingly the non-deterministic algorithm converges very quickly to its stable estimates (RMSE $\approx 1.2 \mathrm{~m}$ ) just after around 20 loops. Note 


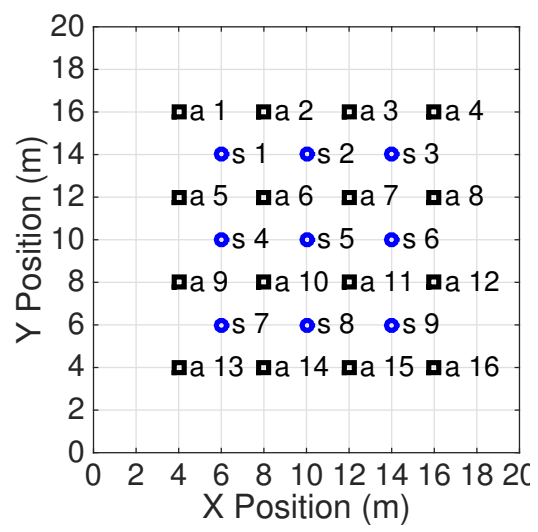

(a)

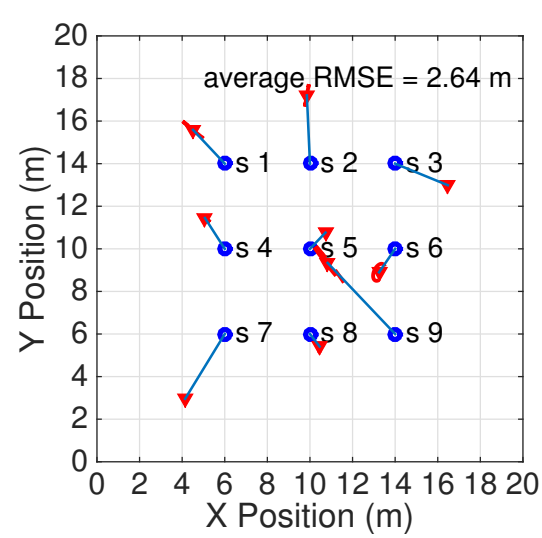

(c)

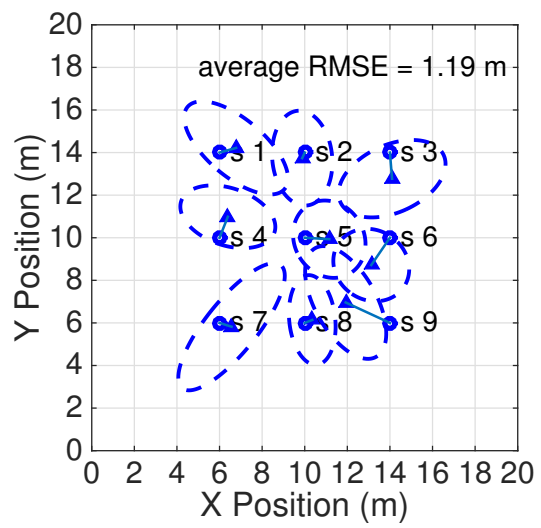

(b)

Figure 5.4: Testbed experiment: (a) 16 Nexus-7 tablets and 9 sound sources marked as " $\square$ " and "o", respectively; (b) estimated locations provided by the non-deterministic algorithm marked as " $\triangle$ "; (c) estimated locations provided by the deterministic algorithm marked as " $\nabla$ ". Dashed ellipses represent the bounds of possible location estimates.

that the deterministic version also requires similar number of loops to converge to its best estimates (RMSE $\approx 2.7 \mathrm{~m}$ ).

Varying the total number of anchor nodes (Nexus-7 tablets), we investigate 


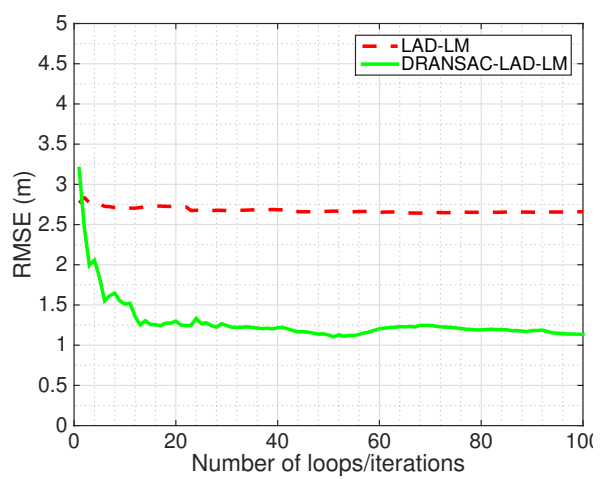

(a)

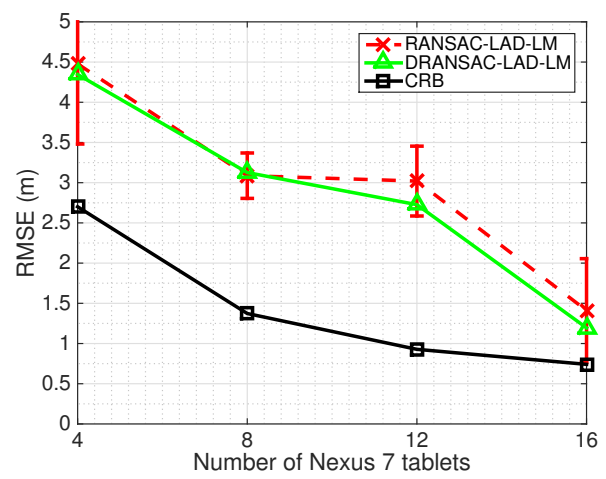

(c)

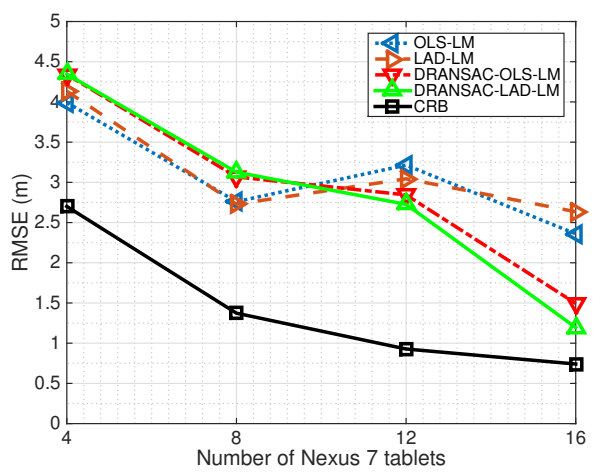

(b)

Figure 5.5: Testbed experiment: (a) Convergence of non-deterministic DRANSAC-LAD-LM vs. deterministic LAD-LM; (b) RMSE when varying the total number of Nexus-7 tablets; (c) Compared with RANSAC-like scheme through 100 runs, DRANSAC-like scheme provides smaller std. of the final estimates.

the performance of the LM technique when being used in different localization schemes. Overall, the RMSE of the algorithms decreases when increasing the number of the devices as shown in Fig. 5.5(b). In particular, the LM using the DRANSAC-like scheme shows its advantage when having sufficient anchor number ( $>12$ anchors) that would enable efficiently iterating the subsets of anchors to remove outliers. Fig. 5.5(b) also shows that our proposed 
DRANSAC-LAD-LM can achieve closely to the CRB when using 16 Nexus-7 tablets.

In addition, we compare the conventional RANSAC-like scheme to our distributed version that uses the Levenberg-Marquardt method to optimize the LAD cost function. The results plotted in Fig. 5.5(c) show that our distributed version is more reliable than the conventional one. The reason is that DRANSAC uses the deterministic model fitting, based on estimated areas and mean value. Conversely, the conventional RANSAC uses line fitting on a subset of estimated positions, which is non-deterministic. Although we did fine-tune parameters of RANSAC line fitting, the RMSEs of RANSAC are still higher than our DRANSAC, which does not require tuning parameters.

Last but not least, we emphasize that the results used to plot graphs in Fig. 5.5(b) and 5.5(c) are optimally obtained from all combinations from the collection of 16 Nexus-7 tablets' positions. The corresponding optimal combinations of four, eight, and twelve Nexus-7 tablets are illustrated in Fig. 5.6(a), 5.6(b), and 5.6(c), respectively. Fig. 5.6 indicates that anchors should be placed symmetrically and towards the bound of the areas of interests in order to obtain the highest localization accuracy.

\subsection{Summary}

In this chapter, we have proposed the DRANSAC scheme, a distributed version of random sample consensus, to deal with uncertainties in sensory data measured by smartphones. In fact, we improve the accuracy of an estimation by exploiting the benefit of collaborating local smartphones to weed out poor measurements. Using the advantage of numerous smartphones in crowded places, the algorithm locally divides measurements into subsets. By doing so, some subsets may comprise either good measurements or poor measurements. Applying a simple rule based on assumptions and constraints of the problem, such as the boundary of the interesting area, we can select the good measurement subsets as the input of an estimator.

Without loss of generality, we apply DRANSAC to the sound localization problem, which is considered as one of the best study cases in terms of nondeterministic effects on data processing. We conduct a testbed experiment with 16 Nexus-7 tablets. The results show that our non-deterministic algorithm gives a RMSE of $1.19 \mathrm{~m}$, which is close to the CRB. The RMSE of DRANSACLAD-LM is two times better than when using its deterministic version, which was considered as an optimal estimator for nonlinear problems by most exist- 


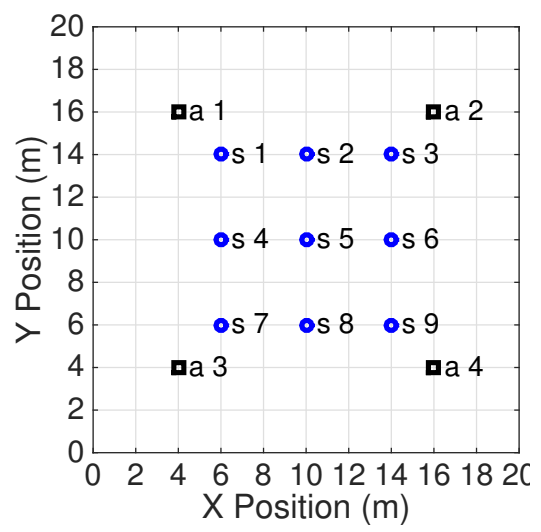

(a) Optimal 4-anchor placement.

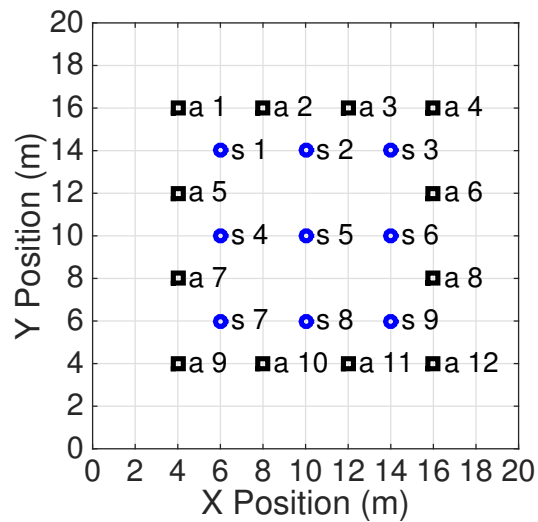

(c) Optimal 12-anchor.

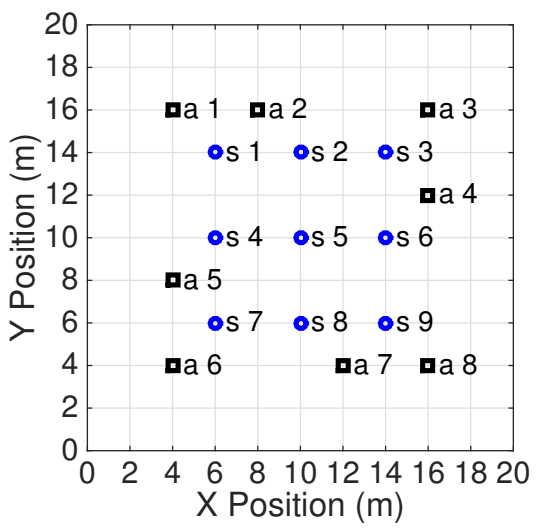

(b) Optimal 8-anchor placement.

Figure 5.6: Optimal placement of anchor subsets obtained by trying all possible combinations with the testbed experiment. Nexus-7 tablets and 9 sound sources marked as " $\square$ " and "०", respectively.

ing work. 



\section{Robust Data Dissemination}

In previous chapters, we introduced distributed sampling and processing approaches to retrieve the information of events. To make use of the retrieved information, smartphones need to broadcast their estimated results to other civilians and governmental departments of the city, for example, the information of a fire or traffic accident. In this chapter, we propose solutions for the data dissemination stack. The solutions include new human-like models and algorithms for message routing in Heterogenous Sensor Networks (HSNs). Through realistic simulations and an experimental dataset, we show that our proposed approaches are more practical and perform better than most existing ones. Moreover, we study that using short-range wireless interfaces such as WiFi and Bluetooth on smartphones to broadcast messages throughout a small city like the Enschede is feasible. When supported with Road Side Units (RSUs) that are placed on main roads of the city of Enschede, more than $80 \%$ of the messages can be delivered to the datacenter at the central bus station of the city within 10 minutes.

This chapter is partially based on:

- V.-D. Le, H. Scholten, and P. Havinga, "Evaluation of opportunistic routing algorithms on opportunistic mobile sensor networks with infrastructure assistance," International Journal On Advances in Networks and Services, vol. 5, no. 3 and 4, pp. 279-290, 2012.

- V.-D. Le, H. Scholten, and P. Havinga, "Towards opportunistic data dissemination in mobile phone sensor networks," in Proc. of The Eleventh International Conference on Networks (ICN 2012), 2012.

- V.-D. Le, H. Scholten, and P. Havinga, "Unified routing for data dissemination in smart city networks," in Proc. of the 3rd International Conference on the Internet of Things (IoT2012), 2012.

- V. D. Le, H. Scholten, P. Havinga, and H. Ngo, "Location-based data dissemination with human mobility using online density estimation," in Consumer Communications and Networking Conference (CCNC), 2014 IEEE 11th. IEEE, 2014, pp. 450-457. 


\subsection{Introduction}

One of the common approaches to disseminate messages is through a standard channel such as cellular networks, 3G mobile internet, or WiFi networks. However, not all smartphones have a mobile internet subscription. Even if a smartphone has a subscription, the connection may not always be available due to the connection fee, and mostly being disconnected when a disaster happens, for instance, an earthquake, criminal sabotage, or tsunami. But mobile phones still have the means to participate in exchanging messages through onboard short-range radio interfaces such as WiFi and Bluetooth. Routing algorithms using the store-carry-forward paradigm have been proposed in a number of recent studies to evaluate the performance of routing algorithms on data gathering [88-101]. However, these algorithms use either basic scenarios or simple simulation architectures that are still quite far from real-world applications as in our study $[102,103]$.

Towards providing a practical solution, this chapter presents approaches for data dissemination, exploring and exploiting the available short-range radio interfaces and mobility of smartphones to provide confident and robust routing in a smartphone-based sensing network, characterized by contemporaneous end-to-end connectivity. In particular, we investigate the performance of existing opportunistic routing algorithms for Delay/disruption Tolerant Networks (DTNs) by proposing a heterogeneous architecture, mobility models, and routing algorithms. The architecture includes most real-world sensor nodes such as RSUs, buses, cars, and pedestrians with unpredictable movements. To achieve a realistic setting, the architecture is mapped onto a real city, the city of Enschede, the Netherlands. The buffer size and the time-to-live (TTL) of messages are limited. We also consider heterogeneous means of communication, especially WiFi and Bluetooth. In addition, we propose new mobility models for pedestrians, cars, and RSUs. These models, together with available ones in The ONE simulator [104], are implemented to make the simulation more realistic. The new models are evaluated through an experimental dataset. Moreover, we propose two message dissemination algorithms. The former deals with the diversity of heterogeneous network. The latter copes with the contemporaneous end-to-end connectivity due to human mobility. By means of simulations, the proposed architecture, models, and algorithms are evaluated and compared with other opportunistic routing protocols.

The remainder of this chapter is organized as follows: Section 6.2 presents a brief survey on current opportunistic routing algorithms. The mobility models are presented in Section 6.3. Next, we propose two new routing approaches in 
Section 6.4 and Section 6.5. Finally, Section 6.6 concludes this chapter.

\subsection{Opportunistic Routing Approaches}

Conventional routing protocols $[105,106]$ require contemporaneous end-to-end connectivity for a data packet to be delivered. In other words, if the destination is not available on a connected path, the packet delivery will fail and no further effort is taken to secure next transmissions of the data. Consequently, the routing protocols must be adapted for HSNs aforementioned in Section 1.1, which can be characterized by intermittent connectivity and sparse mobility. Numerous opportunistic routing algorithms have been proposed in the last few years using different mechanisms [88-97,99-101,107]. They can be generally categorized based on either the type of the network (without infrastructure and with infrastructure) or the pre-known information of the networks (Stochastic and Context-based) as defined in [108]. These categories slightly overlap each other as depicted in Fig. 6.1. If a network is sparse and most nodes possess unpredictable movement, the stochastic protocols are more appropriate. In our opinion, an algorithm that can combine advantages of stochastic and contextbased approaches is most suitable for our considered HSNs since the global knowledge of the fixed and mobile infrastructure perhaps improves the routing performance of mobile nodes, which have unpredictable movement.

\subsubsection{Routing Without Infrastructure Assistance}

Most stochastic routing protocols deliver messages by simply disseminating them all over the network without infrastructure assistance. Being passed from node to node, messages can be gradually delivered at the destination. Epidemic Routing [94] diffuses messages similar to the way viruses/bacteria spread in biology. When encountering others, a node will replicate and broadcast the messages to them. These nodes that just received the messages will move to other places and continuously replicate and transmit messages to other nodes whenever they are in the range of communication. Though increasing the possibility of message delivery, the method results in flooding the network and rapidly exhausting available resources. Direct Delivery (DD) [95] only delivers the holding messages directly to the destination; therefore, DD saves huge amounts of resources but significantly decreases the delivery probability. Spray and Wait (SnW) [96] is a tradeoff between the multi-copy scheme 


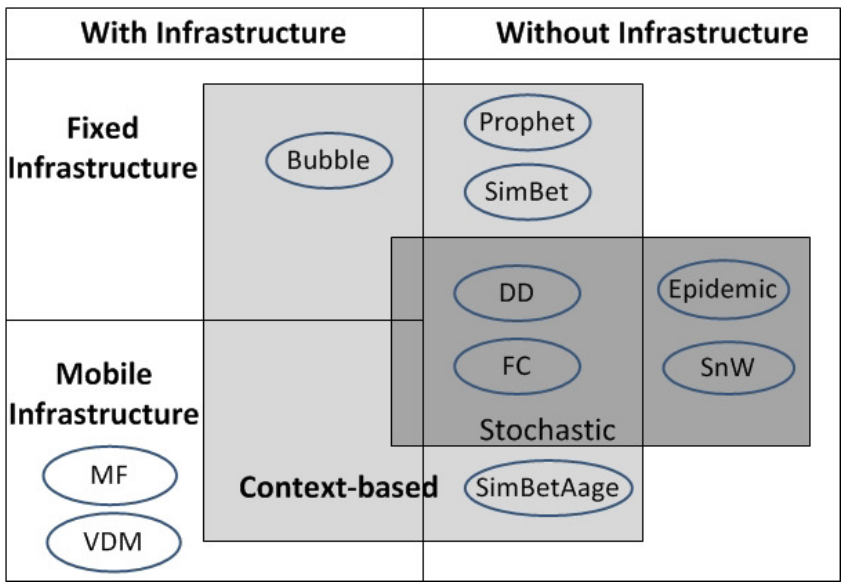

Figure 6.1: Categorizations of routing protocols in opportunistic networks.

(Epidemic) and the single-copy scheme (Direct Delivery) by finding an optimal number of message copies and dividing the message delivery process into two phases (spray phase and wait phase). First Contact (FC) [97] is a variant of the single-copy scheme, which sends messages to the first encountered node or a random node if there are more than one.

Most context-based protocols also do not require infrastructure to disseminate data. In particular, the context-based protocols use the information of historical contacts. Probabilistic Routing Protocol using History of Encounters and Transitivity (PRoPHET) [98] is a well-known context-based routing protocol based on encounter. PRoPHET estimates the delivery predictability for each known destination at each node before passing a message. The estimation relies on the history of encounters between nodes. SimBet [99] uses historical contacts to calculate two metrics, similarity and betweenness. The similarity, which is calculated by how frequently a node and its destination have met, is meant of how socially connected such two nodes are. Meanwhile, the betweenness, which is calculated by how many nodes a node has met, is meant of how interconnected a node is. However, if the utility metrics are equal, SimBet will prevent its forwarding behavior. 


\subsubsection{Routing With Infrastructure Assistance}

Data Mule [109] is designed to exchange messages between the close fixed infrastructure via mobile nodes, which have random movements. Conversely, Virtual Data Mule (VDM) [93], Message Ferrying (MF) and its variants [92,110, 111] try to improve network performance by increasing the encounter probability via predefined movement. The ferries shuttle along the predefined routes in the region of interests. Meanwhile, mobile nodes have the tendency to move towards ferries to send messages. Such an assumption makes the algorithms limited to specific scenarios with majority of buses and bus travellers. In fact, these algorithms are entirely constrained by the route and time schedule of ferries. Without the route information, the algorithms will perform poorly.

To improve the flaw of SimBet, BUBBLE [100] adds the knowledge of community structure to ensure message diffusion. Since the social knowledge varies over time, information used by BUBBLE may be outdated. In addition, the betweenness may be useless if the message is near its destination.

\subsubsection{Routing for Heterogenous Sensor Networks}

To our best knowledge, little attention has been given on how to apply above opportunistic routing algorithms on data dissemination in HSNs. DTF-MSN [107] shows a scheme to gather information in the Delay/Fault-Tolerant Mobile Sensor Network based on an improvement of Direct Delivery and Epidemic. The proposal consists of two key components: queue management and data transmission. Queue management decides the importance of messages, and data transmission decides the node with high delivery probability to send messages to. However, the scenario used to evaluate the proposal has only one mobility model, where both source and sink are mobiles nodes, and is far from realistic for the HSN application domain. Camara et al. [88] present a good mechanism for the distribution of messages, but the mechanism limits itself to vehicle-to-vehicle and infrastructure-to-vehicle. The work uses only the basic Epidemic routing and there is no comparison with other routing protocols. Recently, Keranen et al. [112] evaluate opportunistic networks with various mobility models and routing algorithms by using the ONE. Nevertheless, the used architecture does not include fixed infrastructure and the results only show the simulation speed.

Therefore, we are interested in investigating routing in HSNs, which consist of fixed, mobile infrastructures, and mobile nodes with unpredictable movement. Since algorithms are proposed for different optimization objectives un- 
der different constraints and scenarios, it is difficult to compare the performance of them all. We also improve the ONE simulator for simulations. The ONE includes several opportunistic routing algorithms and mobility models. Researchers can import their own maps and configure the simulator with their own settings by many parameters, such as speed of mobility, message size, buffer size, and etc. Moreover, the ONE is open-source, enabling researchers to develop the tool for their own specific objectives.

\subsubsection{Evaluation Metrics}

Four metrics are used to evaluate the aforementioned performance requirements of different routing algorithms. Two of them are metrics implemented in the ONE: delivery probability and latency. Hop-count metric is no longer an informative metric to assess the delivery cost in time and distance in HSNs as it is used in connected ad-hoc WSNs. Instead, we define Delivery Speed and Delivery Cost for a more accurate evaluation.

- Delivery ratio (DR): The total number of successfully delivered unique messages, denoted by $Q$, divided by the total number of created unique messages, denoted by $P$. Each unique message is created at a certain time, and has a unique identification number to be distinguished from others in the network.

$$
D R=\frac{Q}{P}
$$

- Latency ( $D L)$ : The average of delays between the moment that unique message $i$ is originated, denoted by $T s_{i}$, and the time when the first replicate of unique message $i$ arrives at the destination, denoted by $T d_{i}$. The replicate is a copy of the unique message. The number of replicates depends on the methodology of the DTN routing algorithm, single or multiple-copies.

$$
D L=\frac{1}{Q} \sum_{i=1}^{Q}\left(T d_{i}-T s_{i}\right) .
$$

- Transmission cost (DC): The total number message transmissions, denoted by $T$, divided by the number of successfully delivered messages.

$$
D C=\frac{T}{Q} .
$$


To evaluate the proposed architecture and the proposed mobility model, we use the inter-contact time, first defined by Chaintreau et al. [113]. Intercontact time is the time interval between two successive contacts of a pair of nodes, from the end of one contact to the next contact with the same node. Inter-contact time represents the frequency of opportunities for nodes to send packets to other nodes. The distribution of inter-contact time has an impact on the performance of different routing algorithms. [113] also shows that the intercontact times are power-law distributed with the power-law exponent less than one.

\subsection{Mobility Models}

In this section, we introduce mobility models that can be used in a simulation for data dissemination in a city through heterogeneous networks. The models are also investigated given not only simulation data but also against real-world measurement data.

\subsubsection{Human-like Mobility}

To increase the realism of the mobility model, we propose two additional models, Random Shortest Path Map Based Movement (RSPMBM) and Road Side Unit Placement (RSUP). The new models, together with existing Map Based Movement (MBM), Bus Traveler Movement (BTM), and Bus Movement (BM), are suitably applied for different types of sensor nodes. This approach represents the heterogeneous nature of reality, with RSUs, cars, buses, and pedestrians.

We assume that a portion of mobile nodes represents pedestrians wandering around without any specific purpose. The existing MBM provided by the ONE is likely the most suited. MBM is the Random waypoint movement with map-based constraints, in which a mobile node moves from one map node to another by selecting a neighbouring map node randomly. This movement is repeated a randomly chosen number of times.

Naturally, people do not just wander around. They want to go somewhere for a purpose, using the shortest or fastest path possible. The choice of walking or taking the car is often decided by the Euclidean distance to the destination. These destinations are very diverse [114], ranging from points of interest in the public domain (e.g. restaurants, parks, offices) to more private ones (e.g. friends, home, family). Therefore, we propose a new mobility 
model, RSPMBM, to model the behaviour of human-like mobility. A node selects an arbitrary destination within a predefined range and then moves along the shortest path. Euclidean distance ranges are configurable in a setting file, for example, the distance ranges can be set $[50,500]$ and $[500,5000]$ meters for pedestrians and cars, respectively. Remark that the minimum walking distance of a pedestrian is set to $50 \mathrm{~m}$ to ensure every node always travels a little.

It is reasonable to assume that a number of civilians, called bus travellers, prefer traveling by bus. Movements of bus travellers and buses are modelled by Bus Traveler Movement and Bus Movement, which are also available in the ONE simulator, respectively. A bus traveler compares distance to the nearest bus stop with to the destination to decide whether to take a bus or not. A bus can carry many passenger and shuttles following its pre-defined route and timetable.

The new RSUP model is proposed for deploying RSUs on a map, along side roads with a certain distance between each other. The RSUs are stationary and form a wireless ad-hoc network or wireless sensor network.

\subsubsection{Model Evaluation}

In order to evaluate our proposed architecture and mobility models, a realistic simulation environment is set up using a real city map. The results from running selected routing protocols are analyzed and compared to gain a better understanding of the performance of the existing routing protocols. From that, we may attain implications for future work. We use the ONE simulator [104] that is specially designed for opportunistic networks. It allows users to import maps, configure radios, message size, node speed, etc. The ONE is the fact that it is open-source, so we can flexibly develop new features for better simulations. Indeed, we conduct our simulation on a realistic map, the Enschede city as shown in Fig. 6.2. The red squares $\square$ are RSUs fixed on main roads, and the blue circles o represent mobile nodes including pedestrians, cars, and busses. The green star $\star$ at the center is the common sink.

Fig. 6.3 plots the complementary cumulative distribution (CCDF) of the inter-contact times. The graphs show that the inter-contact time distribution of RSPMBM has a power-law distribution with the exponent approximate 0.3 and similar to the real iMote trace [115]. This power-law distribution does contradict the exponential decay implied by previous mobility models that have been used to design routing algorithms (see [113]). Because the exponent and shape of the distribution may vary between environments, we did not configure parameters to produce the exact same exponent and shape as the iMote 


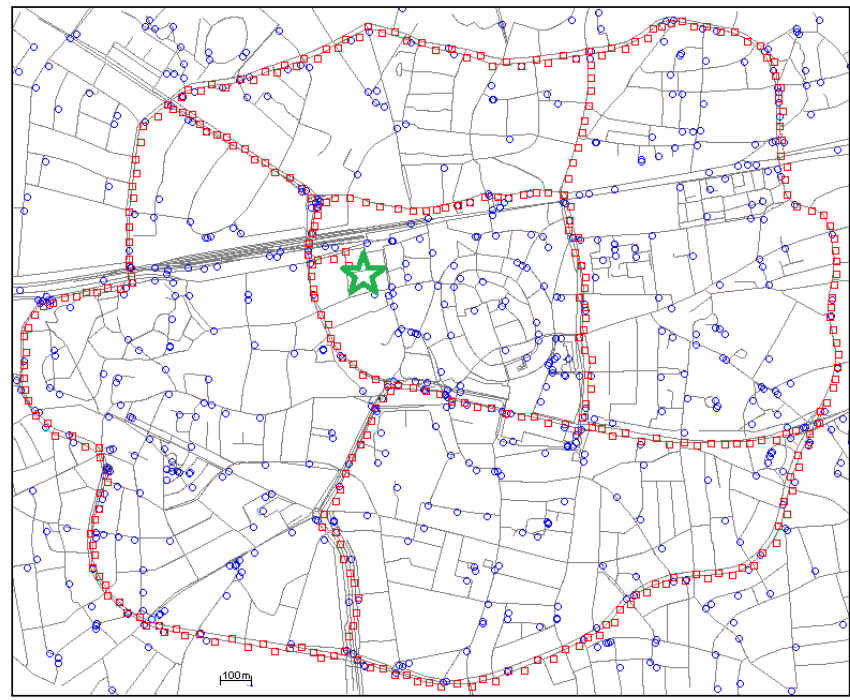

Figure 6.2: Simulation on the city map of Enschede.

trace. Note that the match between the iMote trace and RSPMBM in the first two-thirds of the graph. The difference in the last part of the graph is due to the longer trace (in time) of the iMote, leading to more contacts with low distribution probabilities. RSPMBM has shorter contact times due to the RSU communication backbone. In other words, nodes in our simulation environment meet one another more frequently that those in the iMote experiment.

Fig. 6.3 also shows the inter-contact time distribution for MBM used in the Enschede City Scenario (ECS) for comparison. Surprisingly, both RSPMBM and MBM produce similar tails of the distribution (exponent coefficients are about 0.3). However, the inter-contact time distribution of RSPMBM has a higher probability than that of MBM. This is expected; inter-contact times usually get shorter with increasing reality [104].

\subsection{Unified Routing for Heterogeneous Networks}

As discussed in Section 6.2, current routing algorithms cannot perform well in the aforementioned HSNs. Therefore, it is necessary to have a unified routing 


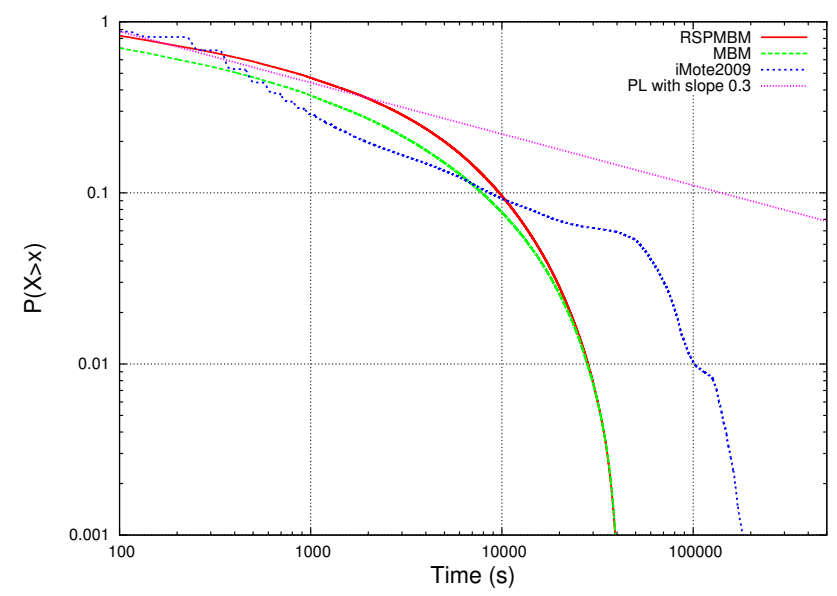

Figure 6.3: Inter-contact times for RSPMBM compared to the iMote trace.

algorithm that adopts most of the components of heterogeneous architecture such as lampposts, pedestrians, cars, and buses. RSUs are intentionally designed in order to improve not only the reliability of event detection, but also to play an important role in both gathering and disseminating data. Since the RSU network is a connected ad-hoc network with a few constraints on throughput, energy, and storage capacity, the approach should preferably transfer messages along the RSU lines rather than among mobile nodes to a common sink, such as the base station shown in Fig. 5.2. The proposed scheme must also allow messages to be temporarily stored at RSUs so that other mobile nodes can directly retrieve the necessary information.

Moreover, the algorithm should avoid flooding the mobility network since mobile nodes have limited power and bandwidth, while still obtaining a high delivery ratio. It is necessary to disseminate a detected event to some specific areas or groups. Normally, the people surrounding an event are interested in what is happening nearby because it is likely that they are at risk. Therefore, the approach should not entirely flood the whole city with such information, but only neighboring areas. Finally, the algorithm must be light-weight and need as little network information as possible because mobile phones have limited energy and computation power, and many people are unwilling to share their personal information.

Following on from the above, we define the desirable objectives of our pro- 
posed routing algorithm for data dissemination in smart city networks:

- must work well with a heterogeneous architecture

- maximize the use of the RSUs' network to improve performance in terms of delivery ratio and latency

- control flooding phenomena in the mobility group

- perform better than existing algorithms

- possibly manipulate disseminated-message areas

- need as little network oracle as possible to facilitate implementation in real-world applications.

To achieve these goals, we propose the Unified routing algorithm, combining the advantages of stochastic and oracle-based algorithms. By adding mechanisms to vary two parameters, namely the Delivery Capability and the Number of Copies, the algorithm can adapt itself to respond to various node and connection types.

\subsubsection{Unified Parameters}

Unified uses the Delivery Capability and the Number of Copies to decide which node and which message to start transferring, respectively. The Delivery Capability value is a constant, whereas the Number of Copies gradually decreases from an initial value.

\section{Delivery Capability}

The Delivery Capability, denoted by $\sigma$, represents the possibility that a node can deliver information based on its hardware characteristics. For example, a RSU usually has the unlimited power supply, high bandwidth, and thus possesses a high Delivery Capability value. Otherwise, a pedestrian carrying a mobile phone which has battery constraint and low bandwidth will be assigned a low Delivery Capability. Since the hardware is almost the same for all nodes in the same component and remains constant over a long period, nodes in the same component have the same value of the Delivery Capability, and nodes from different components certainly own different values of the Delivery Capability. 
When there are several nodes in communication range, a node will be selected as a master node and the remaining nodes as slave nodes by applying some existing clustering mechanism, such as K-means clustering. The master uses the value of the Delivery Capability to determine which slave node to transfer messages first. The slave node, of which the Delivery Capability is higher than that of others, will have the higher priority to exchange messages with the master. This connection selecting is essential to assure that messages are handed to the most suitable nodes since the cluster will not last for long due to the fact that nodes move continuously.

\section{Number of Copies}

The Number of Copies, denoted by $L$, defines the constraint of message reduplication. Depending on the type of network and the number of sensor nodes, all messages originating from the same component have the same initial value of the Number of Copies. The values will be gradually decreased down to 1 by dividing by 2 after each successful transfer.

Unlike the Binary Spray and Wait mechanism proposed in [96], which does not allow a node to send out a message that has the Number of Copies equal to 1 and is consistent with the maximum instants of a message in the network, Unified still allows a node to transfer such a message to other nodes if these nodes belong to different components. Under such circumstances, a receiver will check the original component of the incoming message, from which component the message was created. If the message was generated by a node in the same component, the receiving node will assign 1 to the Number of Copies of the message in order to deter further broadcasting. If not, the initial Number of Copies of the receiving node will be attached to the message to encourage more dissemination. This idea is based on a hypothesis: Information is well-known within a component but unpopular in other components.

In fact, the Number of Copies is used to optimize the delivery ratio of messages. If the Number of Copies is too small, there will be few opportunities to deliver messages to their destinations. However, if the Number of Copies is too large, many copies will flood the network and decrease the delivery performance.

\subsubsection{Unified Routing}

Each component $k$, such as RSUs or pedestrians, has its own predefined the Number of Copies, denoted by $L_{k}$, and the Delivery Capability, denoted by 
$C_{k}$. Clearly, the values of $L_{k}$ and $C_{k}$ rely heavily on the physical characteristics of each component. Moreover, these parameters can be flexibly set to match specific architectures and applications. To this end, we are led to the flowing pseudo code of the Unified routing algorithm for an arbitrary master node $i$. A master is the node is holding the communication channels as a cluster head, and trying to transmit its messages to the in-range neighbouring nodes.

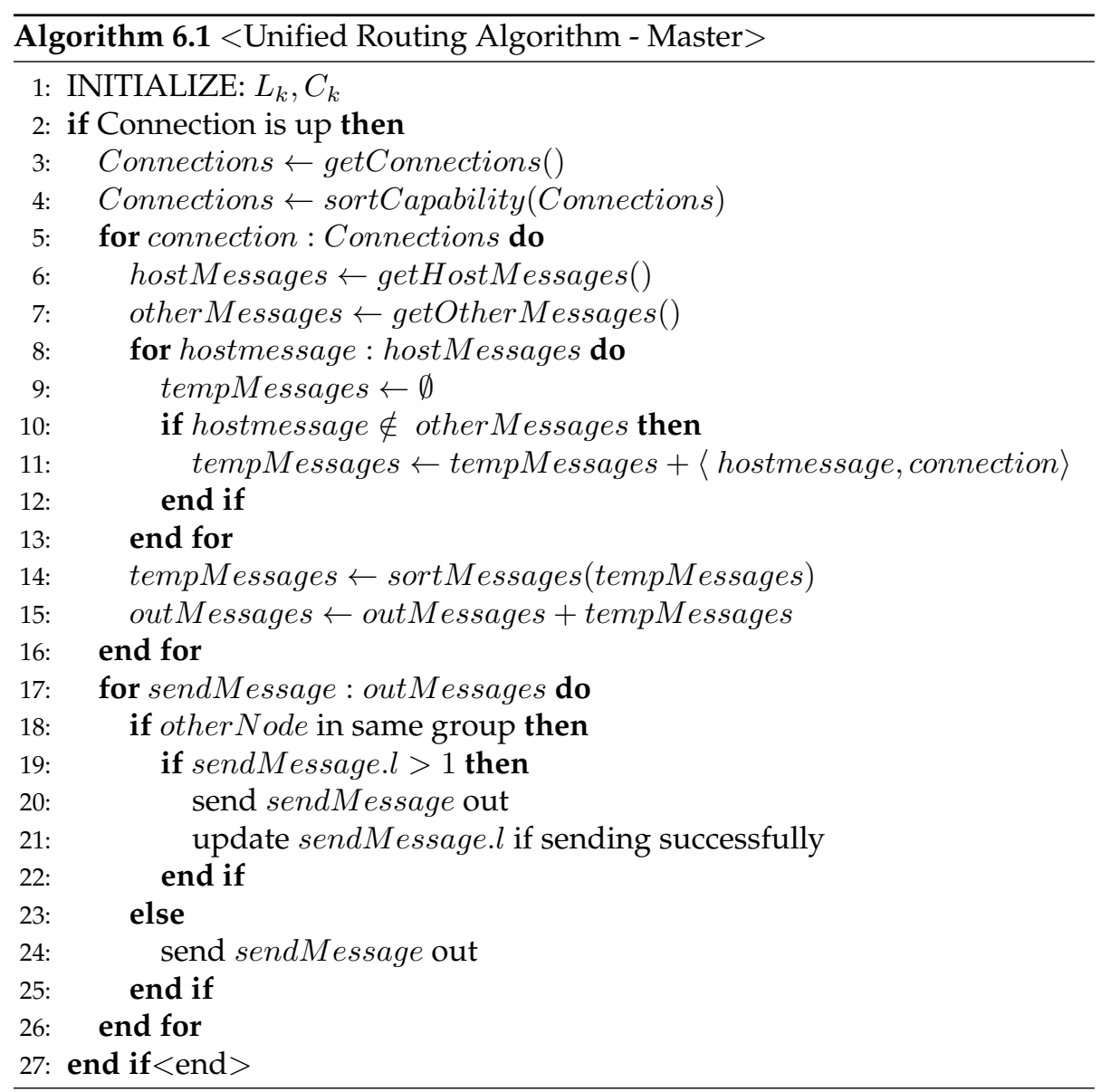

When node $i$ encounters one or more nodes, the connection is up, the master collects and sorts all active connections in descending order of the Deliv- 
ery Capability $C_{k}$ by sortCapability(Connections). Then, for each connection, two nodes exchange their summary vectors to determine which messages the other node does not own, similarly to Epidemic [94]. These requested messages are sorted by a message sorting method, such as random or First In First Out (FIFO), before being pushed into the outgoing buffer outMesssages. From now, node $i$ can start sending out selected messages through connection by connection. Note that the current Number of Copies, sendMessages.l, is reduced if and only if the receiving node is in the same group. A slave node receiving messages will check again whether received messages exist in its buffer. The current Number of Copies $l$ of recevingMessage will be updated as the rule defined in Section 6.4.1. As long as a received message comes from a node in the same component, the slave node updates the current Number of Copies recevingMessage.l by dividing by 2 . Once the sending node belongs another component, the receiving node will check the origin of the message. If the message originates from a node in the same component, recevingMessage.l will be set to 1 . If not, recevingMessage.l is set as $L_{k}$ of the slave node. Algorithm 6.2 describes the pseudo code of messages received at a slave node.

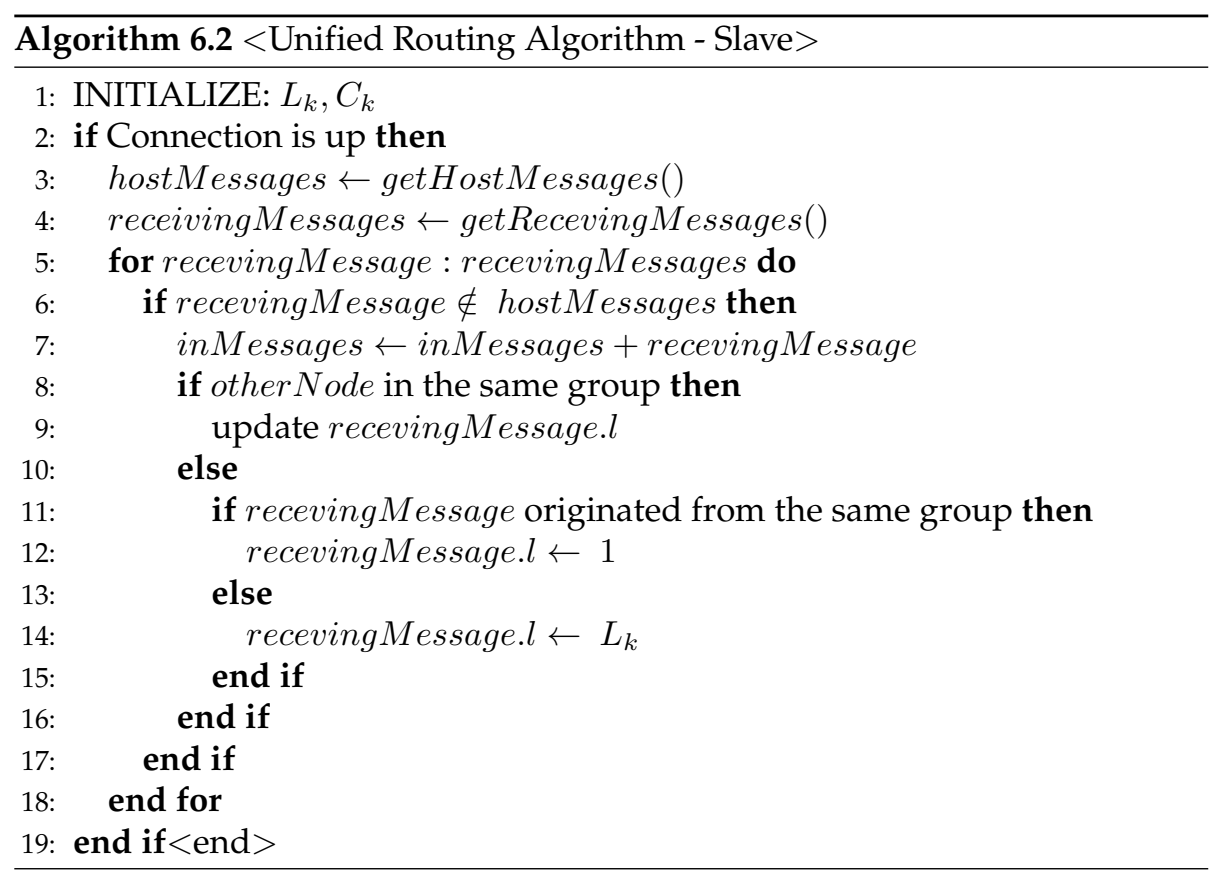


In fact, the Unified algorithm also covers most novel existing schemes, both stochastic and oracle-based routings. When $L$ is set as infinite for all components, the algorithm switches itself to an Epidemic-like scheme by entirely flooding the network with message copies. When $L$ is finite, a node will spread messages like Spray and Wait $(+\infty>L>1)$ or Direct Delivery $(L=1)$ to nodes inside the component, and like Epidemic to nodes outside the component. Furthermore, the predictability of delivery, which can be estimated by an oracle-based routing algorithm, can be used to sort the requested messages of each connection at line 14 of Algorithm 6.1. Since messages probably have various destinations, the predictabilities of a node to deliver messages are not unique. Therefore, those messages that possess higher delivery probability should be transferred before others.

\subsubsection{Optimizing $C$ and $L$}

Without loss of generality, we analyze our proposal with one single city only for convenience of understanding. It is possible to extend the approach for the multiple-city scenarios by adding the Message Ferries algorithm for the bus component. Thus, busses are categorized into the mobility component, and we consider the optimal values of $C$ and $L$ for only two components in our proposed algorithm: Road Side Units and Mobility.

\section{Road Side Units}

RSU networks are a kind of Wireless Ad-hoc Network (WAN), so one might think conventional routing algorithms would be the best options. In fact, the aim of conventional routing is mainly to find the shortest or least-cost path for message delivery. However, the prime objective of data dissemination is not only delivering messages to a specific sink, but also broadcasting them as widely as possible to warn other civilians about imminent dangers. Under such circumstances, conventional routing algorithms fail to spread information over broader areas. Conversely, the proposed Unified with setting high $C$ and large $L$ can work well.

On the one hand, the Unified approach transfers messages along RSUs to a sink quickly since it is not necessary to find a shortest path. Since RSUs are implemented in linear formation, a RSU can only communicate with at most two neighbors. Once a message first reaches a RSU, no more than two copies of a message are created. Afterwards, the algorithm replicates the message copy and send it to the next RSU. In this way, the message is rapidly delivered to the 
sink. The progress of the Unified routing does not take much longer than that of conventional algorithms because there are only a few branches or spanning trees that lead to the sink.

On the other hand, this approach causes the RSU network to be entirely flooded with messages. Once a RSU receives a message from outside of its network, for instance, from a pedestrian, Unified speedily diffuses the message to most of the RSUs. Other pedestrians, cars, or even buses can retrieve the information directly from RSUs they encounter. Therefore, it is unnecessary to send a request message to the base station to demand information. In other words, RSUs can be either sink or source nodes for the mobile sensor network.

To this end, the optimal value of $C$ for RSUs should be set to the highest value $(C=1)$ so that messages can take the advantage of the RSU network. In addition, the optimal value of $L$ should be infinite $(L=+\infty)$ in order that messages can reach as many RSUs as possible.

\section{Mobility}

We consider a sparse mobility network in which pedestrians are the majority. Limited battery, narrow throughput, low speed and unpredictable movement patterns self-evidently imply that the mobility component has a fairly low delivery capability. Therefore, the optimal value of $C$ should be the lowest: $C \simeq 0$. In addition, the $L$ of mobility can be in the range from 1 to $+\infty$ depending on the expected delivery ratios and mitigation of network-flooding effects. However, one of the simplest ways to find a near optimal value of $L$ for mobility is to solve the equation of mitigation of the trade-off between optimal and expected latency, described in [96], by letting $E D_{s w}=a E D_{o p t}$, where $a$ is a factor for the delay constraint dictated by the application. Similarly to the numerical example in [96], we set $a$ to 5 . This means the expected delay is allowed to be up to five times of the optimal delay.

The expected delay of the optimal case, when $L=+\infty$ :

$$
E D_{o p t}=\frac{H_{M-1}}{M-1} E D_{d t}
$$

where $H_{n}=\sum_{i=1}^{n} \frac{1}{i}$ and $M$ is the number of mobile sensor nodes.

The upper-bound of the expected delay of normal case with $1<L<+\infty$ message copies is:

$$
E D_{s w} \leq\left(H_{M-1}-H_{M-L} E D_{d t}+\frac{M-L}{M-1} \frac{E D_{d t}}{L}\right) .
$$


Suppose that $1<L \ll M$, we have $H_{M-1} \approx H_{M-L} \approx H_{M}$ and $M-$ $1 \approx M-L \approx M$. Therefore, $\mathrm{L}$ in Equations 6.5 can be simplified as:

$$
L \approx \frac{M}{a H_{M}},
$$

Analogously, we can set other values of $C$ and $L$ for a sub-mobility group, for example, cars. Cars usually have high-speed and long-range communication so they certainly possess higher $C$ than pedestrians do. In fact, the more appropriate value setting of these two parameters, the better performance of networks.

We remarked earlier that there is another solution to find the optimal Number of Copies for the mobility component in a heterogeneous architecture as Fig. 5.2. In fact, Equations 6.4 and 6.5 are derived on the assumption that the network has only mobile nodes and the destination of a message is a random mobile node. However, our considered network is heterogeneous with various types of components, including stationary and mobile nodes. Furthermore, the comment destination is a common sink fixed in the center of the map. Therefore, the expected delay of $L=+\infty$ and $1<L<+\infty$ cases will be slightly different. We are going to investigate this aspect in a journal since it takes a lot of space to describe the solution. However, the estimated value of $L$ for mobility calculated by applying Equation 6.6 is almost consistent with our simulation results.

\subsubsection{Simulation}

We first define setup for our simulation with regard to HSNs. Then we compare our proposed algorithm with other algorithms when performing in a realistic scenario.

\section{Simulation Setup}

The simulation uses the center of the city of Enschede as a realistic setting. In the center of the map, there is the central bus station. The map shown in Fig. 6.2 takes up approximately 3000 by 3000 meters and is exported from Openstreetmap.org. We create several submaps for RSUs, roads for cars, paths for pedestrians, and routes for busses. There are 336 RSUs deployed in linear formation at the outer ringroads, inner ringroads, and four radiating roads leading to the center of the city. Pedestrians are able to roam freely, but cars can 
only run on roads. Moreover, four distinct bus lines in the city are mapped onto the simulation. For each bus line, two buses are assumed to serve the route.

Since our main concern is the contribution of pedestrians to data dissemination, the number of pedestrians will be varied between 258 and 1058. One thousand of pedestrians seems too few, but is in fact realistic because the population density of Enschede is just about $1000 / \mathrm{km}^{2}$ and we assume that roughly one-tenth of the civilians walk in streets at a certain time. The simulation also includes 50 cars. The initial positions of cars and pedestrians are randomly distributed. The speed of cars and pedestrians are randomly generated in ranges of from 10 to $40 \mathrm{~km} / \mathrm{hr}$ and 1.8 to $5.4 \mathrm{~km} / \mathrm{hr}$, respectively. Since pedestrians walking at almost uniform speed form the majority of the network, the effect of the mobility velocity on performance results is not studied in this simulation.

As mobile internet and mobile services are expensive, we only consider built-in short range interfaces, such as Bluetooth and WiFi. We assume that all mobile phones possess Bluetooth Version 2.0 at $2 \mathrm{Mbit} / \mathrm{s}$ net bit rate with a $10 \mathrm{~m}$ radio range, while only smartphones have a WiFi interface at net bit rate of $11 \mathrm{Mbit} / \mathrm{s}$ with a $60 \mathrm{~m}$ radio range. According to Eriksson et al. [116], the $11 \mathrm{Mbit} / \mathrm{s}$ rate overall performs much better than the other $802.11 \mathrm{~b} / \mathrm{g}$ rates for vehicles. Fifty percent of pedestrians own smartphones, and the rest uses regular phones without the WiFi interface. RSUs have both interfaces as do smart phones. However, cars and buses possess WiFi only because Bluetooth is unrealistic at high speeds.

Our proposed RSPMBM is applied for most pedestrians and all cars with travel distances ranging from 50 to $500 \mathrm{~m}$ and 500 to $5000 \mathrm{~m}$, respectively. Cars certainly can go farther than $5000 \mathrm{~m}$, but they would be out of the simulation area of $3000 \mathrm{~m}$ by $3000 \mathrm{~m}$. In the RSPMBM model, a pedestrian would walk for a short distance, typically $50-500 \mathrm{~m}$, but not usually for long distances such as $500-5000 \mathrm{~m}$. For a long distance, he or she would tend to drive a car. In addition, we assume that there are always 100 pedestrians who just stroll around the city and modeled by the MBM [104]. These pedestrians simply follow Random Walks along map paths. Modeled by the BM model, buses shuttle between the bus stops along fixed routes. Finally, among pedestrians, we assume that there are 100, who prefer taking the bus and are modeled with the BTM model [104]. They walk to a nearby bus stop and wait to catch a bus to take them to their destinations.

During the interval time, which is randomly drawn from 25 to 35 seconds, an event happens at a random place in the city. Nearby mobile phone sensors, which are carried by pedestrians or cars, will measure data together. A node will be assigned as a cluster head that is responsible for distributed data pro- 
cessing to detect such event. A message, which contains inferred information about the event, is created at the cluster head and ready for dissemination. In other word, there will be 732 unique messages created during simulation of 6 hours in real world. In our study, RSUs do not generate messages, but act as a communication backbone. In fact, we intend to use RSUs to further improve the reliability and accuracy of detection in future. For message size setting, as [117] shows that the optimal message size for MAC layer is approximate 500 bytes, a random size between $0.5-1$ Kbytes is assigned to each message. We remark that 500 bytes suffice for most kinds of data in a sensor network such as, humidity, temperature, or toxic chemical intensity. Since the common buffer length for the MAC layer is from 25 to 50 packets, the message buffer is set at 25 Kbytes for all nodes in most of the simulations in this paper, except when studying the effects of varying the buffer size.

Data dissemination is simulated using our proposed algorithm. The Delivery Capability $C$ of RSUs is set as one, and that of mobility is set as zero. The Number of Copies $L$ of mobile nodes are varied from $1 \ldots 10^{9}$. Meanwhile, $L$ of RSUs are set as infinite for all cases. Furthermore, a comparison with First Contact, Spray and Wait, Epidemic, and ProPHET is also made. For the same reason as mentioned in [103], Message Ferries (MF) is not used for buses in our simulation since MF is more suitable for carrying messages from city to city.

\section{Results and Discussion}

Using the above configured scenario, we evaluate performance of the Unified algorithm in terms of average delivery ratio, latency, transmission cost. FIFO is used to sort messages, in line 14 of Algorithm 6.1. The compared algorithms also used FIFO to sort message buffers to make a fair comparison. The results demonstrate that the Unified algorithm performs significantly better others in most cases.

Fig. 6.4.a plots the overall delivery ratio of the Unified algorithm when the Number of Copies $L$ of mobile nodes increases from 1 to $1 e+9$. There are 658 mobile nodes.By benefiting from the RSU network, Unified can obtain quite high delivery ratios even when $L=1$, at least $47.81 \%$ with $T T L=60$ minutes. The delivery ratios are significantly improved when $L$ changes from 1 to 5 since there are more copies broadcast. However, the delivery ratios remain constant and even decrease a little if $L$ increases continuously, especially with large $T T L$. Large $L$ and $T T L$ cause the flooding effect similarly to that for Epidemic. In addition, Unified gives high delivery ratios with short TTL messages because the algorithm can drop more expired messages, which probably 


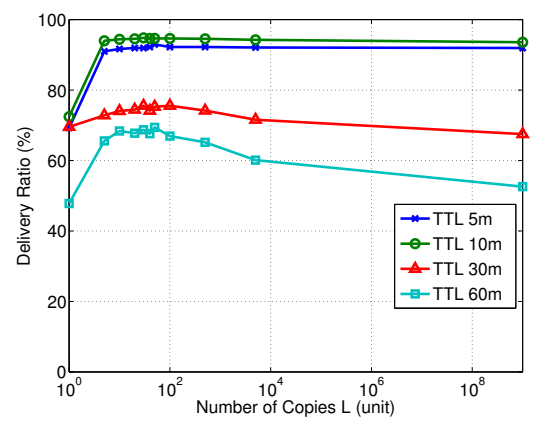

(a)

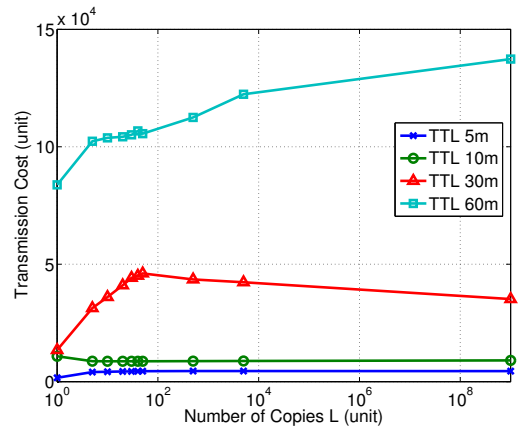

(c)

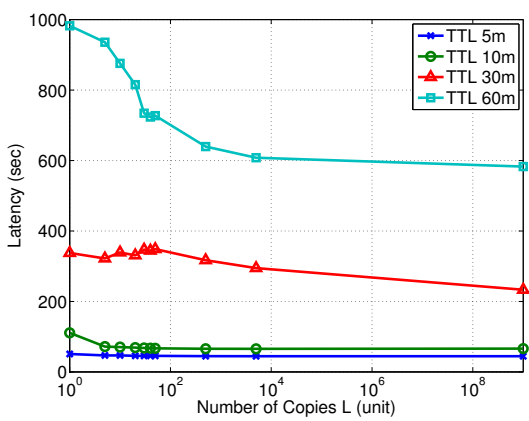

(b)

Figure 6.4: Unified performance with varying the number of copies

have already been delivered, allowing it to receive new ones.

Fig. 6.4.b shows the average latency of message delivery performed by the Unified algorithm. The algorithm can obtain good delivery delay for messages with short $T T L$ even with a small Number of Copies $L$. However, for large $T T L$, Unified has to run with $L=20$ at least to have good latency for $T T L=60$ minutes. By looking at the delivery cost shown in Fig. 6.4.c, the total number of messages including replicates over that of delivered messages, we can see that setting $L$ correctly minimizes the tradeoff between latency and transmission cost. It seems that $L=20$ is an optimal value so that the Unified algorithm obtains a high delivery ratio, low latency, and acceptable transmission cost for various values of $T T L$. By considering Equation $6.6, L=20$ is consistent with $L \approx 19(a=5$ and $M=658)$.

We also compare the Unified algorithm with First Contact (FC), Spray and 


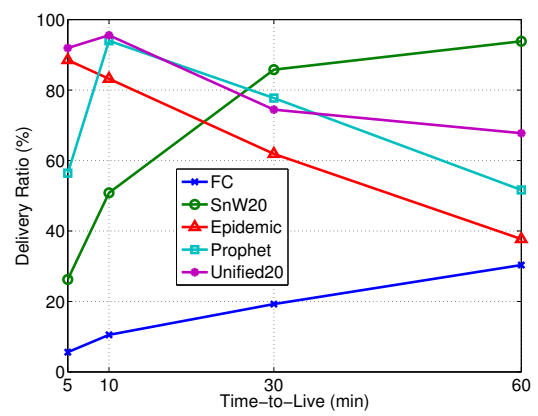

(a)

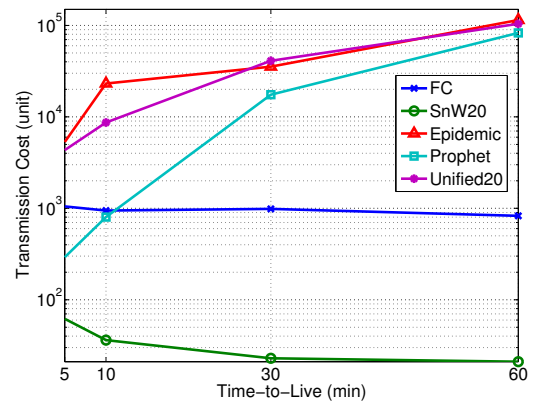

(c)

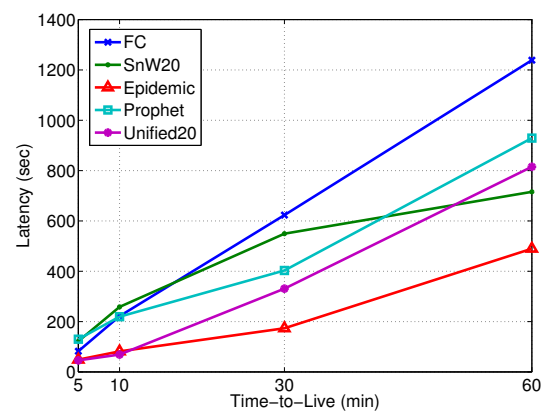

(b)

Figure 6.5: Algorithms comparing

Wait with the number of copies is 20 (SnW20), Epidemic, and ProPHET in Fig. 6.5.a. All algorithms run with the same 658 mobile nodes and 25 Kbytes buffer size. Delivery ratios obtained by applying Epidemic and ProPHET significantly decreases when $T T L$ increases, but SnW20. Observation shows that Unified takes advantage of Epidemic of Epidemic when $T T L$ is short, and that of Spray and Wait when $T T L$ is long. First Contact, of course, gives a very poor delivery ratio because it has only one copy of messages.

Fig. 6.5.b shows that the Unified algorithm has the second lowest average latency because it can take advantage of the RSU network. Clearly, by flooding the network with multiple copies of messages, Epidemic has optimal latency. Overall, larger $T T L$ results in a longer delay since messages can stay longer in the buffer before being delivered. In addition, transmission costs of algorithms 


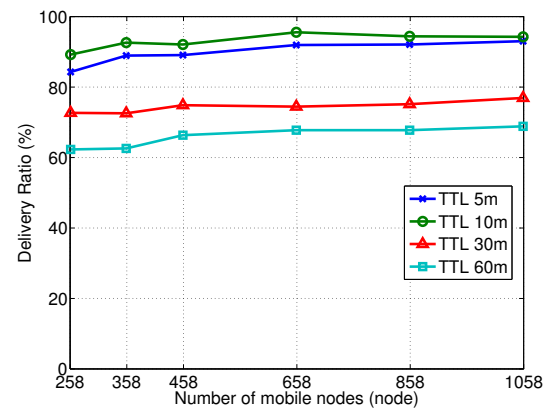

(a)

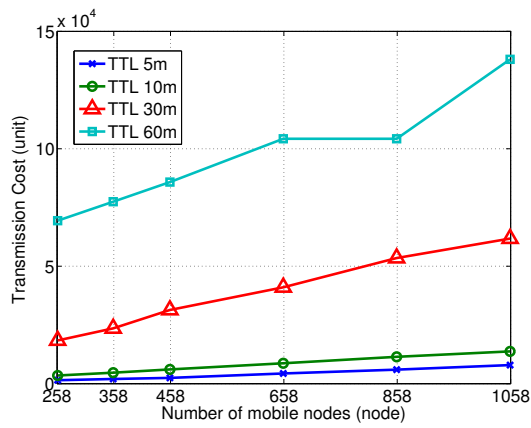

(c)

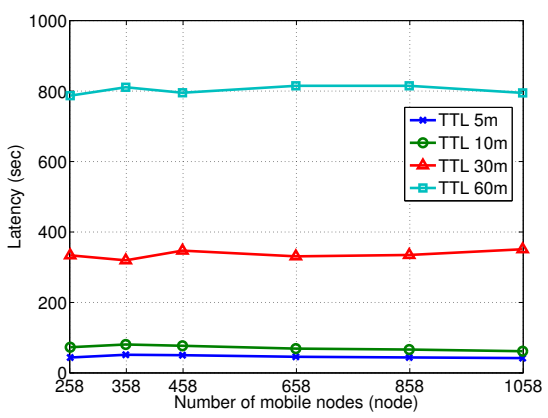

(b)

Figure 6.6: Unified performance with varying the number of mobile nodes

are shown in Fig. 6.5.c. The transmission cost of Unified is as high as that of Epidemic when TTL is greater than 30 minutes. Since our main objective is disseminating information, high transmission cost is not always a drawback since more messages are broadcasted.

To investigate the effect of the network size, we vary the number of nodes but keep the buffer size constant at 25 Kbytes. Results show that the network size does not significantly affect the performance of the Unified algorithm, as shown in Fig. 6.6. Even the number of nodes vary widely from 258 to 1058, the existence of the RSU network makes that delivery ratios and latency almost remain constant in Fig. 6.6.a and 6.6.b for various TTLs, respectively. Only transmission costs monotonically increase with the number of mobile nodes. This is expected since the more nodes, the more transmissions.

Fig. 6.7 shows that the performances of Unified increase significantly in 


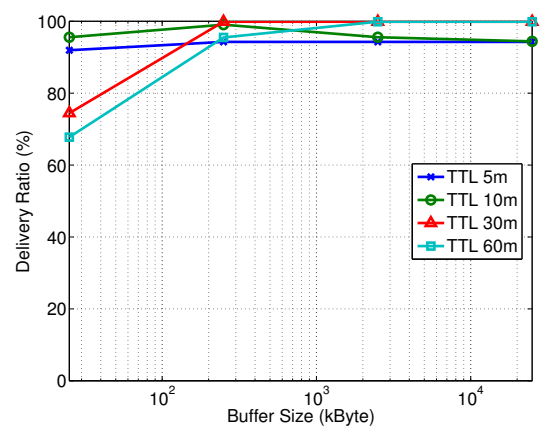

(a)

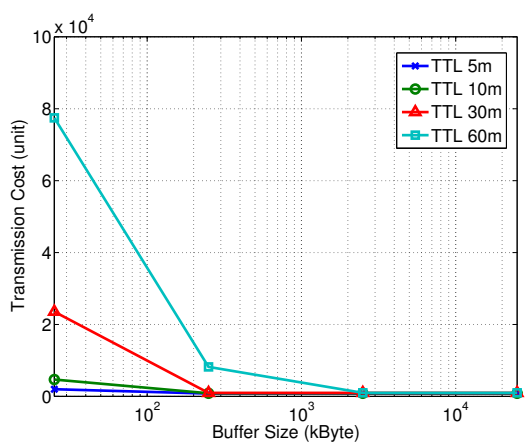

(c)

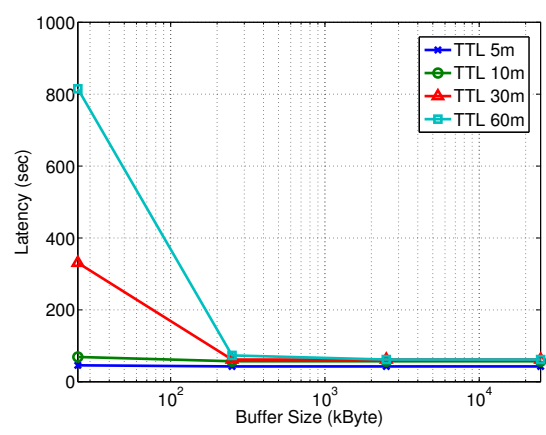

(b)

Figure 6.7: Unified performance with varying the buffer sizes

terms of delivery ratio, latency, and transmission cost when the buffer sizes are bigger since more messages can be exchanged during a limit contact time. However, when the buffer size is greater than 250 Kbytes, holding approximately 375 packets, saturation is reached so that increasing the buffer size hardly improves the performance, as expected. Even with a finite buffer size, a pair of nodes will not be able to exchange all messages in buffers due to limited contact duration. We remark that the optimal value of buffer sizes depends on the mobility model as long as different models have different contact times. As discussed in [103], the Random Shorted Map Based Movement is quite realistic. Therefore, the results shown in Fig. 6.7 are realistic and give a good prediction for real experiments in the future. Note that we set the number of mobile nodes as 658 for all simulations in Fig. 6.7. 


\subsection{Location-based Routing with Human Mobility Behaviours}

In this section, we exploit the impact of human mobility on message delivery in opportunistic mobile phones sensor networks. In particular, we consider a DTN of human-centric nodes. Conventional DTN's routing protocols have attempted to find the probability of message delivery based on contact times, which indicate how frequently a pair of devices is in connection. Though having showed good performance on message delivery, contact-based algorithms do not perform well when each device frequently appears at different regions, as most people daily do. The reason is that geographic coordinates of nodes have little or no correlation with their contact times. For example, assume that a staff currently has a bundle of messages to send to colleagues either working in or out of his office. Since devices of staffs working in the same office have high contact times, most contact-based algorithms estimate delivery probability of roommates of the staff much higher than that of colleagues in other offices. Therefore, the staff preferably transfers messages to his roommates first, and to a visiting person later. As a visitor just drops in for a while, he or she would not have enough time to wait to receive the messages from the staff. As a result, the staff misses a good opportunity to deliver messages to other offices.

Although several contact-based algorithms also consider transitive probability, such as Probabilistic Routing Protocol using History of Encounters and Transitivity (PRoPHET) [98] and MaxProp [118], these algorithms will fail in the case that the mobile phone of a visitor is frequently out of communication range with other mobile phones in his office. This can happen if the visitor is unwilling to participate in dissemination. However, if the message is carried by the visitor, its information can be delivered to the destination by other communication channels because they are in the same office.

The above scenario points out the limitations of current routing approaches based on contact times, and motivates us to propose a new approach based on historical locations of mobile nodes, called location-based routing. The key insight is that nodes frequently appearing nearer the message destinations are regarded as possessing higher delivery probability. In our approach, at regular time intervals, each node records its current location in a first-in-first-out (FIFO) buffer, which has an ageing parameter deciding its length. If the buffer overflows, the oldest location will be removed to make room for a new recorded location. An appropriate unsupervised learning or clustering algorithm will be applied to infer the full information of location densities from recorded data. 
When having obtained the information, we estimate the distribution of nodes in the past to select better candidates to carry messages. To our best knowledge, this is the first work to formulate Opportunistic Mobile Sensor Network (OppMSN) routing problem as an online unsupervised learning problem on historical locations to predict message delivery probability of mobile phone users.

\subsubsection{Problem Formulation}

In this section, we formulate two routing problems in spatial and spatiotemporal domains. The problem in the spatial domain is simpler but less reliable than that in the spatiotemporal domain. In addition, storing both time and coordinates of mobile phones carried by users requires a more sophisticated security scheme to protect private data. Depending on the requirements of applications, researchers can select the suitable domain to apply.

\section{Spatial Domain}

We consider a network of $n$ mobile sensor nodes, denoted by the set $\mathcal{S}=$ $\left\{s_{1}, \ldots, s_{n}\right\}$, which have unpredictable moving patterns. For each node $i$ (i.e., $s_{i}$, used interchangeably) let $\mathcal{D}_{i}=\left\{\boldsymbol{x}_{i, 1}, \ldots, \boldsymbol{x}_{i, k}\right\}$ denote the set of its $k$ most recent locations recorded with time interval $\Delta$. With a slightly abuse of notation, denote the set of encountered nodes of $s_{i}$, including itself, as $\mathcal{E}_{i}=$ $\left\{s_{i}, s_{i+1}, \ldots, s_{i+e}\right\}$, where $e$ is the number of nodes currently connected to $s_{i}$. The set of location history induced by the set $\mathcal{E}_{i}$ is defined as $\mathcal{D}_{i}^{e}=\left\{\mathcal{D}_{i}, \mathcal{D}_{i+1}\right.$, $\left.\ldots, \mathcal{D}_{i+e}\right\}$. The set of $l$ messages which is being held by node $s_{i}$, and needs to be delivered, is denoted as $\mathcal{M}_{i}=\left\{\boldsymbol{m}_{1}, \ldots, \boldsymbol{m}_{l}\right\}$. Each message might have various attributes such as destination, time-to-live, message size, message priority, etc. The problem is for each node to decide which node in its encounter set is the best next message carrier for each message so as to quickly and reliably deliver the message.

We propose a probabilistic framework to solve the above problem. For each message $\boldsymbol{m}_{j} \in \mathcal{M}_{i}$, the destination coordinate is the only relevant attribute concerned in this paper to decide a successful delivery, which in turn depends on the set $\mathcal{D}_{i}$. Other attributes can be used to sort $\mathcal{M}_{i}$ in advance by a buffer management $[119,120]$. By assuming $\mathcal{D}_{i}$ is parameterized by a vector of unknown parameters $\boldsymbol{\theta}_{i}$, the delivery probability can be defined as the probability density function $p_{i, j}\left(\boldsymbol{m}_{j} \mid \boldsymbol{\theta}_{i}\right)$ conditioned on the parameter vector $\boldsymbol{\theta}_{i}$. Here we use a shorthand notation $p_{i, j}\left(\boldsymbol{m}_{j} \mid \cdot\right)$ to denote the event of a successful delivery of 
message $\boldsymbol{m}_{j}$. The set of delivery probabilities of all nodes in $\mathcal{E}_{i}$ for message $\boldsymbol{m}_{j}$ is

$$
P_{i, j}=\left\{p_{i+k, j}\left(\boldsymbol{m}_{j} \mid \boldsymbol{\theta}_{i+k}\right)\right\}_{k=0}^{e} .
$$

Let $\boldsymbol{\Theta}_{i}^{e}=\left\{\boldsymbol{\theta}_{i}, \boldsymbol{\theta}_{i+1}, \ldots, \boldsymbol{\theta}_{i+e}\right\}$, the set of unknown parameters for the nodes in $\mathcal{E}_{i}$. The set of all parameter vectors at all nodes is called location distribution, $\boldsymbol{\Theta}=\left\{\boldsymbol{\theta}_{1}, \ldots, \boldsymbol{\theta}_{n}\right\}$. Our first goal is to estimate the set of parameters $\boldsymbol{\Theta}$ using the location history at all nodes, $\mathcal{D}=\left\{\mathcal{D}_{1}, \ldots, \mathcal{D}_{n}\right\}$. Once the estimate $\widehat{\boldsymbol{\Theta}}$ (and hence $\left.\widehat{\boldsymbol{\Theta}}_{i}^{e}\right)$ is available, each node $s_{i}$ can calculate the set of delivery probabilities $P_{i, j}$ for each message $\boldsymbol{m}_{j}$. Subsequently, by making pairwise comparisons, node $s_{i}$ will find a candidate with higher delivery probability and not holding the message to transfer.

Let us reconsider the problem of learning location distributions from historical data unlabelled to which geographical regions. These data sets may be geometrically viewed as clouds of points in a $d$-dimensional space $(d=2,3)$. Finding a location distribution in our approach is a typical unsupervised learning and clustering problem. A location distribution of node $i$ generally falls into two categories, a single distribution or a mixture of $z_{i}$ distributions. In essence, given historical-location sets $\mathcal{D}$, we have to find the estimate $\widehat{\boldsymbol{\Theta}}=\left\{\widehat{\boldsymbol{\theta}}_{1}, \ldots, \widehat{\boldsymbol{\theta}}_{n}\right\}$ of $\boldsymbol{\Theta}=\left\{\boldsymbol{\theta}_{1}, \ldots, \boldsymbol{\theta}_{n}\right\}$. In particular, given historical location set $\mathcal{D}_{i}$ of node $i$, the problem is to find the estimate $\widehat{\boldsymbol{\theta}}_{i}=\left\{\widehat{\boldsymbol{\theta}}_{i, 1}, \ldots, \widehat{\boldsymbol{\theta}}_{i, z_{i}}\right\}$ of full parameter $\boldsymbol{\theta}_{i}=\left\{\boldsymbol{\theta}_{i, 1}, \ldots, \boldsymbol{\theta}_{i, z_{i}}\right\}$. Then, we can estimate the delivery probability of node $i$ to deliver a message $\boldsymbol{m}_{j}$ by

$$
\widehat{p}_{i, j}\left(\boldsymbol{m}_{j} \mid \boldsymbol{\theta}_{i}\right)=\max _{k=1, \ldots, z_{i}}\left\{p_{i}\left(\boldsymbol{m}_{j} \mid \omega_{i, k}, \widehat{\boldsymbol{\theta}}_{i, k}\right) P_{i}\left(\omega_{i, k}\right)\right\},
$$

where $P_{i}\left(\omega_{i, k}\right)$ is the prior probability of each class with state of nature $\omega_{i, k}$. This is a simplified solution to the mixture of distribution, in which we put all weight on the single best distribution. Finally, for each message $\boldsymbol{m}_{j}$, node $i$ selects the next carrier $s_{c}(i, j)$ to deliver the message as the one with highest estimate delivery probability,

$$
s_{c}(i, j)=\arg \max _{k=0, \ldots, e}\left\{\widehat{p}_{i+k, j}\left(\boldsymbol{m}_{j} \mid \boldsymbol{\theta}_{i+k}\right)\right\} .
$$

\section{Spatiotemporal Domain}

Now we seek to formulate the above problem in spatiotemporal domain. At a time slot $t$ during a cycle of $\mathcal{T}$ time slots, which can be a day, a week, or a month, let $\mathcal{D}_{i}^{t}=\left\{\boldsymbol{x}_{i, 1}^{t}, \ldots, \boldsymbol{x}_{i, k}^{t}\right\}$ denote the set of node $i$ 's locations recorded at 
time slot $t$ of $k$ most recent cycles. The set of location history induced by the set $\mathcal{E}_{i}$ becomes a matrix $\mathcal{D}_{i}^{e}=\left\{\mathcal{D}_{i}^{t}, \mathcal{D}_{i+1}^{t}, \ldots, \mathcal{D}_{i+e}^{t}\right\}_{t=1}^{\mathcal{T}}$. Besides the destination coordinate, the expected delivery time attribute needs to be considered to estimate the delivery probability of node $i$ to deliver a message $\boldsymbol{m}_{j}$. Therefore, the delivery probability of node $i$ to delivery message $\boldsymbol{m}_{j}$ on expected time slot $t$ is defined as the probability density function $p_{i, j}^{t}\left(\boldsymbol{m}_{j} \mid \boldsymbol{\theta}_{i}^{t}\right)$ conditioned on the parameter vector $\boldsymbol{\theta}_{i}^{t}$, which parameterizes $\mathcal{D}_{i}^{t}$. For time slot $t$ in spatiotemporal domain, equations (6.7), (6.8) and (6.9) can be rewritten as

$$
\begin{gathered}
P_{i, j}^{t}=\left\{p_{i+k, j}^{t}\left(\boldsymbol{m}_{j} \mid \boldsymbol{\theta}_{i+k}^{t}\right)\right\}_{k=0}^{e}, \\
\widehat{p}_{i, j}^{t}\left(\boldsymbol{m}_{j} \mid \boldsymbol{\theta}_{i}^{t}\right)=\max _{k=1, \ldots, z_{i}}\left\{p_{i}^{t}\left(\boldsymbol{m}_{j} \mid \omega_{i, k}^{t}, \widehat{\boldsymbol{\theta}}^{t}{ }_{i, k}\right) P_{i}^{t}\left(\omega_{i, k}^{t}\right)\right\},
\end{gathered}
$$

and

$$
s_{c}^{t}(i, j)=\arg \max _{k=0, \ldots, e}\left\{\widehat{p}_{i+k, j}^{t}\left(\boldsymbol{m}_{j} \mid \boldsymbol{\theta}_{i+k}^{t}\right)\right\} .
$$

Note that if one can solve the problem in spatial domain, a similar solution can be applied to the problem in spatiotemporal domain by drawing historical locations regarding the expected delivery time slot.

The computational complexity depends on online machine learning algorithm. For example, the Location-Mean to be described in Section 6.5.2, only needs $O(n)$ to update parameters of $n$ nodes. In spatiotemporal domain, the computational complexity becomes $O(n \mathcal{T})$ with the number of expected time slot bounded by $\mathcal{T}$. The memory cost on each node for the problem depends on dimensions of parameters, which are much smaller than original data.

It is infeasible to compare the complexity between location-based and encounter-based approaches because they are based on two different elements, which are not well correlated. Computational load of the location-based scheme depends on the location updating intervals, which are independent from encounters. The shorter intervals, the heavier computation. Meanwhile, computation load of the encounter-based scheme depends on human mobility and density, which decides how frequently people meet each other.

\subsubsection{Unsupervised Learning Approaches}

For simplicity and clarity, we describe how to estimate the distribution parameters for the spatial domain. The spatiotemporal domain can be solved analogously. Our approach is to use recorded locations to estimate the unknown 


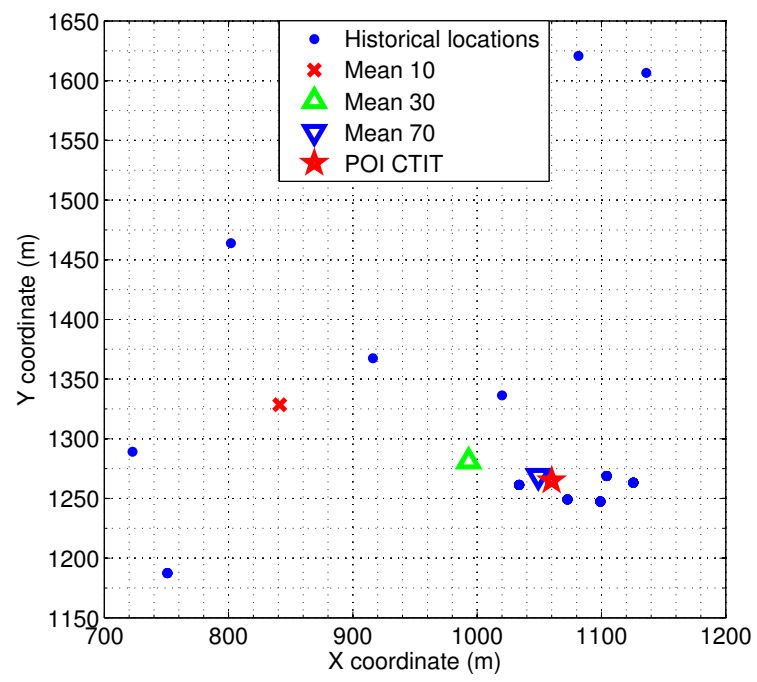

Figure 6.8: Illustration of the Location-Mean approach from simulation data.

location distributions of a single best distribution of the mixture densities. For simplicity, assume that the densities follow a Gaussian mixture

$$
p_{i, j}\left(\boldsymbol{m}_{j} \mid \boldsymbol{\theta}_{i}\right) \sim \aleph\left(\boldsymbol{\mu}_{i}, \boldsymbol{\sigma}_{i}^{2}\right),
$$

where $\boldsymbol{\mu}_{i}$ and $\boldsymbol{\sigma}_{i}$ are vectors of $z$ dimensions, with only $z$ is known as the second case in Table I. The check mark $(\checkmark)$ and question mark (?) indicate known and unknown parameters. Therefore, this problem can be solved by existing classification and clustering tools, such as Gaussian Mixture Model (GMM), Kalman filter, or Support Vector Machine(SVM) [121]. Once the problem is solved, we will obtain estimated prior probability $\widehat{P}_{i}\left(\omega_{i, k}\right)$, mean $\widehat{\boldsymbol{\mu}}_{i}$ and $\widehat{\boldsymbol{\sigma}}_{i}$ vectors of node $i$. Afterwards, the delivery probability in Eq. 6.8 of node $i$ to deliver message $\boldsymbol{m}_{j}$ is computed by

$$
\widehat{p}_{i, j}\left(\boldsymbol{m}_{j} \mid \boldsymbol{\theta}_{i}\right)=\max _{k=1, \ldots, z_{i}}\left\{\frac{\left\|\boldsymbol{m}_{j}-\widehat{\boldsymbol{\mu}}_{i, k}\right\|}{\sum_{k=1}^{z_{i}}\left\|\boldsymbol{m}_{j}-\widehat{\boldsymbol{\mu}}_{i, k}\right\|} \widehat{P}_{i}\left(\omega_{i, k}\right)\right\},
$$

where $\boldsymbol{m}_{j}$ and $\boldsymbol{\mu}_{i, k}$ are also coordinates of the message destination and cluster centers, respectively. 


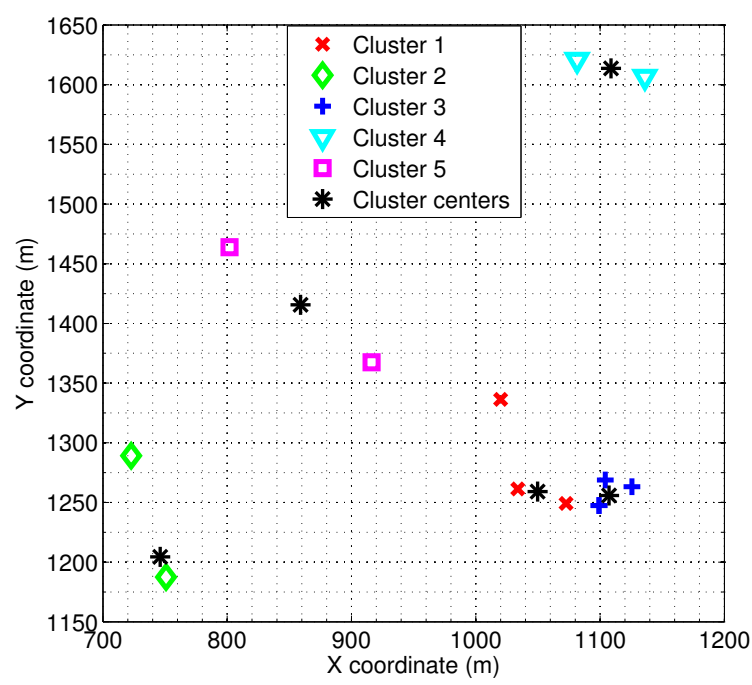

Figure 6.9: Illustration of the Location-Cluster approach from simulation data.

Following on, we present two simple tools to solve the problem of location densities, Location-Mean (mean) and Location-Cluster (k-means) for $k=1$ and $k=z_{i}$, respectively.

\section{Location-Mean}

Table 6.1: Three cases of mixture Gaussian estimation [121]

\begin{tabular}{|c||c|c|c|c|}
\hline Cases & $\boldsymbol{\mu}_{i}$ & $\boldsymbol{\sigma}_{i}^{2}$ & $P_{i}\left(\omega_{i, k}\right)$ & $z_{i}$ \\
\hline \hline 1 & $?$ & $\checkmark$ & $\checkmark$ & $\checkmark$ \\
\hline 2 & $?$ & $?$ & $?$ & $\checkmark$ \\
\hline 3 & $?$ & $?$ & $?$ & $?$ \\
\hline
\end{tabular}

Suppose we knew the distribution of historical locations of node $i$ came from a single normal distribution with a mean $\boldsymbol{\mu}_{i}$ and standard deviation $\boldsymbol{\sigma}_{i}$. Essentially, these two parameters constitute a compact representation of the 
movement pattern. If the mobile phone user actually stays most of the time in a specific place, such as his office building, the historical locations have a mean that tends to fall in the region where the user mostly stays. Of course, if the samples from a user is not normally distributed, the Location-Mean approach can give very misleading description of movement pattern, and the estimate delivery probability will be wrong. At each time $t$, when $\boldsymbol{x}_{i, t}$ is updated, the mean $\widehat{\boldsymbol{\mu}}_{i}$ can be updated incrementally as:

$$
\widehat{\boldsymbol{\mu}}_{i} \leftarrow \frac{N_{i}}{N_{i}+1} \widehat{\boldsymbol{\mu}}_{i}+\frac{1}{N_{i}+1} \boldsymbol{x}_{i, t}
$$

where $N_{i}$ is the current number of historical locations of node $i$.

Fig. 6.8 illustrates the historical coordinates and their means of a node moving according to the movement model to be described in Section 6.5.3. We tested a variety of historical lengths. Means 10,30, 70 are the mean values of the 10,30 , and 70 recorded locations of the node representing a user at the CTIT institute. Because the simulated user spends most of the time in his building, of which the main gate is marked as the star 'POI CTIT', the mean values of 30 and 70 are quite close. This indicates that choosing the length of 30 latest historical locations is sufficient. Note that the time interval we chose to record locations in the simulation is randomly drawn between $250-350$ seconds.

\section{Location-Cluster}

If we consider a longer period of human activity than working hours, the model of a mobile phone user locations is probably a mixture distribution instead of a single normal distribution. We observe that most people still spend most of their time in several places, such as their house, offices, bars, sport centers, etc. Therefore, the normal mixture with an unknown number of class $z$ can give a close description of the location densities, as case 3 in Table I. There are several machine learning methods to estimate the number of classes $z$ of each person. The number of classes $z$ can also be obtained by asking the mobile phone user. Note that each node $i$ has its own value of class number, $z_{i}$.

We use k-means to solve the defined problem since this technique has efficient online update, thus it can simplify the computation and accelerate convergence. In particular, k-means computes the squared Euclidean distances $\left\|\boldsymbol{x}_{i, t}-\widehat{\boldsymbol{\mu}}_{i, k}\right\|^{2}$ at each time $t$ to find the mean $\widehat{\boldsymbol{\mu}}_{i, k}$ nearest to $\boldsymbol{x}_{i, t}$ with $k=1, \ldots, z_{i}$. K-means does not require to know $z_{i}$ in advance; instead, $z_{i}$ can be inferred from the given data. When the distance to $\widehat{\boldsymbol{\mu}}_{i, k}$ is greater than a given threshold $\delta$, we increase $z_{i}$ by one $z_{i}=z_{i}+1$. The mean of the newly created cluster 
is $x_{i, t}$. At each time $t$, when $\boldsymbol{x}_{i, t}$ is assigned to cluster $k$, the mean $\widehat{\boldsymbol{\mu}}_{i, k}$ can be updated incrementally as:

$$
\widehat{\boldsymbol{\mu}}_{i, k} \leftarrow \frac{N_{i, k}}{N_{i, k}+1} \widehat{\boldsymbol{\mu}}_{i, k}+\frac{1}{N_{i, k}+1} \boldsymbol{x}_{i, t}
$$

where $N_{i, k}$ is the current number of data points assigned to cluster $k$. So each node needs to store only a tuple of cluster mean values and their number of assignments, instead of storing the whole data it receives for its whole lifetime.

It is interesting to see how the k-means operates on the example data we used in Fig. 6.8. Fig. 6.9 shows 5 cluster centers and theirs clustered locations. These cluster centers give a compact description of 5 places in campus the node frequently occurs.

We note that there are better Machine Learning tools to solve (6.8), for instance, Maximum Likelihood, Support Vector Machine, Decision Tree, and Gaussian Mixture Model. Some of them may have very high computation that should be considered since mobile phones have limited computation capability and battery.

\subsubsection{Simulation}

In this section, we present our preliminary results, the performance of LocationMean. Note that recent work studying the nature of human mobility [103,112, $113,122]$ has proved that suitable mobility models can sufficiently simulate the behavior of human mobility. A realistic model of human mobility does not mean that the movement pattern is predictable; instead, it better characterises the unpredictable human mobility rather than the simple Random Walk [123].

\section{Simulation Settings}

Our simulation is based on a realistic scenario of the University of Twente campus shown in Fig. 6.10. The routes and Points of Interests (POIs) such as offices, sport centers, stadiums, tennis courts, libraries, restaurants, shops, supermarkets, staff houses, and dormitories are mapped into the simulation. For each place, there is a WiFi access point installed at each main entrance, which is marked as square in Fig. 6.10. These nineteen access points are also the sinks of messages that are randomly generated at one of mobile phones, which are carried by students and staff. We assume that the speed of pedestrians remains almost constant at $0.5-1.5 \mathrm{~m} / \mathrm{s}$. Therefore, the mobility speed has a minor effect on performance results. 


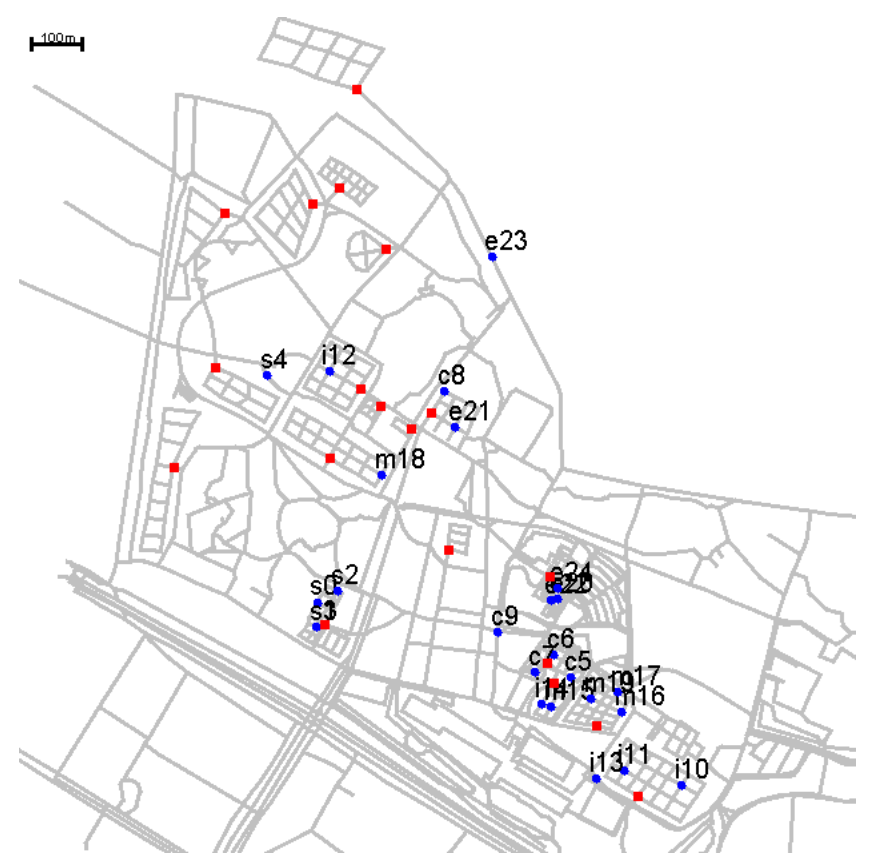

Figure 6.10: Screenshot of simulation. WiFi access points marked as square and pedestrians marked as circles.

The mobile phones and sinks are supposed to possess a WiFi interface at net data rate of $11 \mathrm{Mbit} / \mathrm{s}$ with $30 \mathrm{~m}$ radio range. Every thirty seconds, a new message with size of 500 Bytes to 1 KBytes is created, and its destination is one of the access points. Buffer sizes of mobile phones and sinks are 25 KBytes and 25 MBytes, respectively. The First In First Out (FIFO) is applied on buffer management.

In addition, students and staff are split into 5 sub-groups, called STAFF, CTIT, IMPACT, MESA++, and ELAN as the name of research institutes in University of Twente. Their movements are modeled with the Shortest Path Map Based Movement, which is presented by [104], and various POIs. At a certain moment, a node will choose one of nineteen POIs with predefined probability. In particular, the probability of a node to visit libraries, shops, or sport centers are $5 \%, 10 \%$, and $5 \%$, respectively. Since we concern the effect of humans in 


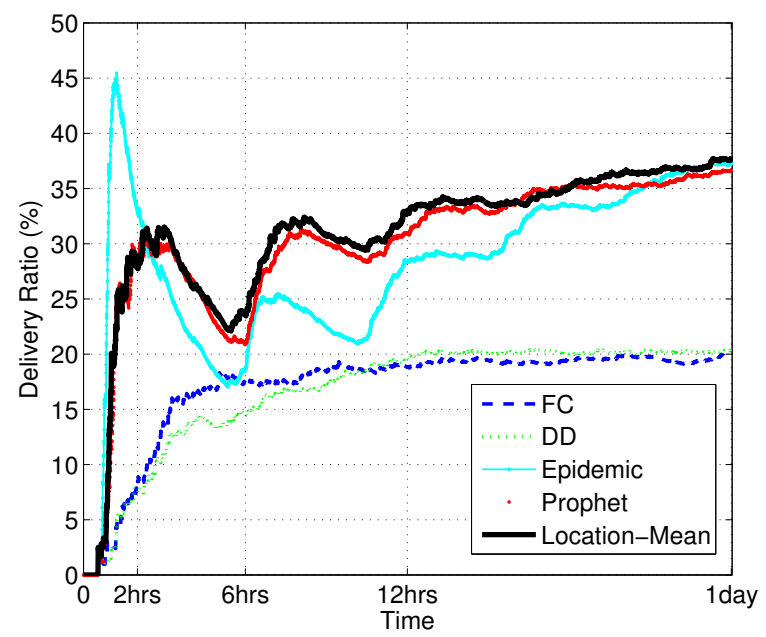

Figure 6.11: Convergence of delivery ratios.

data dissemination during day time, $60 \%$ of the time students and staff stay in their offices, and only $10 \%$ of the time they visit their homes for a while. Moreover, we assume that every 30 to 60 minutes there is at least a person entering or leaving a building in the simulation.

With above settings, our proposed algorithm is evaluated and compared with a number of well-known opportunistic routing protocols: Direct Delivery (DD) [95], FirstContact (FC) [97], Epidemic [94], and PRoPHET [98]. Since Location-Mean is a very naive algorithm as an example for location-based, we do not include results of better contact-based algorithms, such as BUBBLE and SimBetAge, to make a fair comparison.

\section{Simulation Results}

All results are averaged over five runs with difference random seeds to simulate one day in real time. Fig. 6.11 shows the convergence of delivery ratios when time increases. Because of the limited buffer size and contact durations, delivery ratios quickly converge after first two hours. The delivery ratios of DD and FC firmly converge after 12 hrs while those of Epidemic, Prophet and 


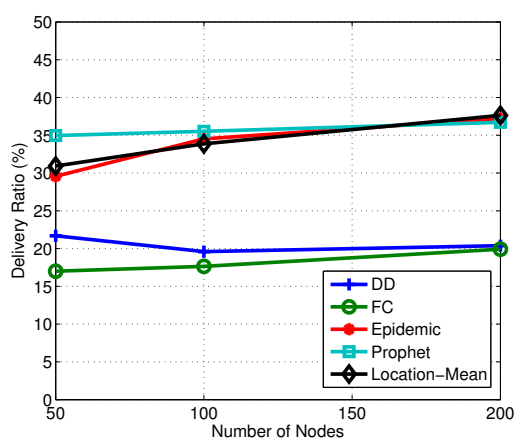

(a) Delivery ratio.

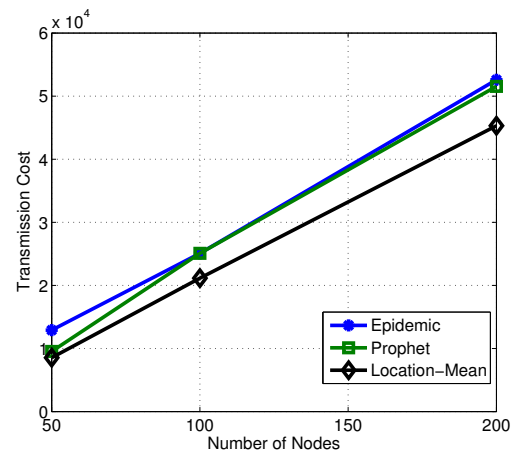

(c) Transmission cost.

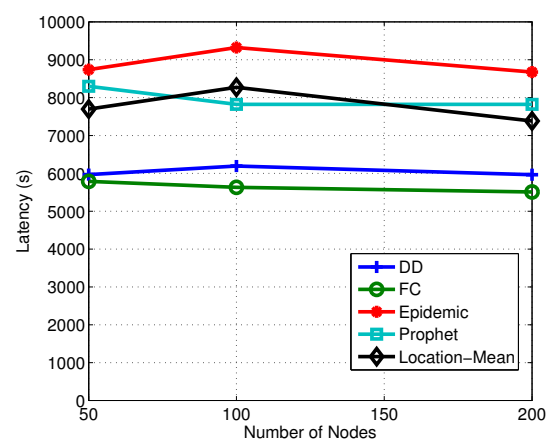

(b) Latency.

Figure 6.12: Performance with varying the buffer number of mobile nodes.

Location-Mean still slightly raise up. We also observe that the delivery ratio given by Location-Mean reaches $41 \%$ after two days and still keep slightly increasing. This is explained by the longer period, the closer estimate the means of the locations.

We also evaluate the delivery ratios of the algorithms by varying the number of participants in the above scenario, from 50 to 200 . We remark that the number of mobile nodes here represents the people moving in and out of the buildings on the campus, not the number of total students and staffs. In such way, 200 can represent 2000 people working on the campus, and thus save a lot of simulation time. 
Fig. 6.12(a) shows the percentages, after one day, slightly rise when the number of nodes increase. This is expected since more mobile nodes produce more contact opportunities. Note that this observed increase is not held with DD because it only sends a message to the destination node. This can be proved using Random Walk theory [123]. Location-Mean scores best among compared algorithms when increasing the number of nodes. This implies that Location-Mean knows better which nodes to transmit the messages than the others, which seem like random dissemination.

We also examine how the Location-Mean performs in terms of latency as shown in Fig. 6.12(b) since the time taken to deliver messages is important. We measure the average delays when changing the number of nodes from 50 to 200 as we evaluate the delivery ratio. Since our algorithms can predict the potential nodes better to avoid the traffic loads, its latency is lower others multi-copies schemes when increasing the number of nodes. We remarked that the average delay obtained by our algorithm, about 120 minutes, is quite long for some applications. However, it makes sense because the delivery totally relies on the walking speed of $4 \mathrm{~km}^{2}$ on the campus, and the users usually stay in either their office or classes. Note that the problem caused by latency can be solved by prioritizing messages based on required delivery time.

We remarked that latency obtained by Epidemic is higher than by Prophet in Fig. 6.12(b) is reasonable. Only under ideal conditions such as unlimited buffer sizes and all messages can be exchanged during any contact duration, Epidemic will give the lowest latency. However, our simulation is set with limited buffer sizes (message queue), contact durations, and very short communication ranges. This makes Epidemic have a longer delay than Prophet, which is consistent with the investigation in [98].

Resource consumption is always a key metric in evaluating routing algorithms in mobile phone sensor networks. Fig. 6.12(c) shows the transmission costs, defined in Section 3, of our proposed algorithm and some existing algorithms. DD has the lowest transmission cost since it only transfers messages to the destinations. FC also has very low transmission cost since it is single-copy routing. Therefore, we subtract them from the plot to have a clearer visualization. Among the multi-copy schemes, Location-Mean has lowest transmission cost as we expected. Since Location-Mean infers movement patterns of nodes based on locations, it hands the messages to better candidates to avoid roaming messages.

In addition, we observed that the simulation of Location-Mean runs faster than that of Epidemic and Prophet with regard to an identical setting, which consists with computation complexity discussed in Section 6.5.1. For instance, 
simulation time for Location-Mean is 3802 seconds with described 200-node scenario, $20 \%$ and $12 \%$ shorter than Prophet and Epidemic, respectively.

\subsection{Summary}

In this chapter, we have proposed solutions for efficient message transmission in HSNs. To save the deployment cost and increase the robustness, we address using off-the-shelf wireless interfaces of smartphones to disseminate messages. To improve the network performance in terms of delivery ratio and delivery latency, we proposed to add extra devices such as RSUs and mobile wireless gateway installed on public transportation. In addition, we also propose two DTN routing algorithms: the unified routing algorithm and the location-based algorithm. The unified routing algorithm explore and exploit the advantage of different kinds of networks to transfer messages as efficient as possible. On the other hand, the location-based algorithm applies online unsupervised learning techniques on the historical locations of smartphones. Given the recent locations, the delivery probability of a smartphone with regard to a destination is estimated through solving a mixture densities problem. Based on the values of delivery probability, smartphones are selected to continue carrying the messages to their destinations.

Through realistic simulation scenarios based on the real maps and mobility models based on the human mobility, the results are consistent with the theoretical analysis of the proposed solutions. This chapter also gives implications for further development of opportunistic routing algorithms with online unsupervised learning and clustering for location densities in both spatial and spatiotemporal domains. With better density parameter estimation methods such as support vector machine or decision trees, we expect the performance to be significantly improved. 


\section{Conclusions}

Throughout previous chapters, this dissertation presents symbiotic sensing solutions that overcome the new challenges in mobile sensing. In Chapter 2, we review the state-of-the-art of smartphone-based sensing systems and their techniques with respect to a number of criteria. In Chapter 3, we present an cooperative distributed hybrid sensing architecture that are inspired by the symbiosis of living beings in nature to share sensing resource and avoid conflicts. The symbiotic architecture is composed of three main stacks: data sampling, data processing, and data disseminating. Our proposed solutions for each stack are consecutively presented in Chapter 4, 5, and 6.

To conclude this dissertation, we first revisit the major contributions of this dissertation in Section 7.1. Next, concluding remarks are discussed in Section 7.2. Finally, we discuss directions for future work in Section 7.3.

\subsection{Evaluation of Contributions}

We proposed and addressed a new symbiotic sensing research problem in the context of Heterogenous Sensor Networks (HSNs) through the contributions that are re-summarized as follows.

\section{Contribution 1: Cooperative Hybrid Sensing}

Data sampling with smartphone platforms is constrained by resources, especially batteries. Therefore, we propose a new bio-inspired sensing strategy called symbiotic sensing to reduce resource consumption in Section 3.2. The new sensing paradigm is well mathematically modelled to quantitatively explore and evaluate the probability of the sensing success. The evaluation results show that the commensal sensing paradigm has merits when there are 
plenty of smartphones to carry out the sensing tasks. To cope with hybrid sensing paradigms including the new symbiotic sensing, we also proposed a possible cooperative distributed sensing architecture in Section 3.3. The architecture provides an outline for designing a new generation of smartphone-based sensing system through three major stacks: the data sampling, the data processing, and the message dissemination. Based on the skeleton of this architecture and techniques presented in later chapters, a smartphone-based sensing system can be developed for specific applications.

\section{Contribution 2: Cooperative Data Sampling}

Most data sampling methods for onboard sensors of smartphones are conducted with a predefined scheduling based on learned behaviours of the contexts and the requirements of the applications. However, we argue that the data sampling must be adaptive to the change of contexts to save the energy consumption and efforts. The fact is that presented solutions in Chapter 4 can meet these challenges. The hypothesis is if sampling scheduling can adapt to the change of the contexts, it will save the smartphone and human resources without loss of the sensitivity or information. Through experimental evaluation, the featherweight context change detection algorithm presented in Section 4.5 evaluates the hypothesis of adaptive sampling. The proposed algorithm is based on the likelihood of historical sampled data to detect the change points of the context. The detected change points are used as triggers to schedule the sampling carried by smartphone sensors. Without loss of generality, we show that our solutions could save at least $80 \%$ resources with the audio context in our experiments. This is a significant improvement compared with existing approaches.

\section{Contribution 3: Non-deterministic Data Processing}

We are among the first to explore the impact of the non-deterministic smartphone-based platforms on sensing, exploiting collaboration to meet the accuracy requirements. The contribution is presented in Chapter 5. Without loss of generality, we investigate key elements that result in inaccuracy measurements for audio data in Section 5.2. From learned models of the non-deterministic measurements, we propose a cooperative distributed approach based on the non-deterministic scheme, which divides measurements into subsets to weed out the outliers. Through the testbed experiment with sound localization, we show that the non-deterministic approach we proposed performs much bet- 
ter than others. The results also confirm our hypothesis; the non-deterministic scheme can deal with the noise included in smartphone-based measurements better than the deterministic scheme.

\section{Contribution 4: Robust Data Dissemination}

In many smartphone-based sensing systems according to our survey in Chapter 2, transmitting data from smartphones to a central server or to other smartphones are via long-range radios such as mobile Internet and WiFi gateways. Only one in fifty uses the short-range radios to transfer sensing data in a delaytolerant network routing scheme. The main reason is that Delay/disruption Tolerant Network (DTN) schemes usually cause a substantial delay and drop the delivery ratio due to the intermittent connectivity. Therefore, we proposed the solutions for such problems in Chapter 6. In Section 6.3, we propose new movement models that imitate the human movements. The models enhance the realism of the DTN simulations, of which real experiments are costly and troublesome to deploy. We also propose two new routing algorithm to overcome the challenge in Section 6.4 and 6.5, respectively. The unified routing algorithm presented in Chapter 6.4 can cope with the diversity of the HSN by exploiting the delivery capability of devices. The location-based routing algorithm described in Chapter 6.5 can deal with the mobility of persons who carry the smartphones by applying machine learning techniques. The simulations show the improvement of the routing performances and promise a potential research direction, using machine learning for DTN routing.

\subsection{Conclusions}

While the first generation smartphone-based sensing systems focus on best effort data collecting, backend and non-cooperative data processing, current proliferation of smartphones has intrigued researchers to focus on sensing performance in terms of power efficiency, reliability, robustness, and short feedback to users. However, designing a smartphone-based sensing system to meet these requirements faces major challenges related to the battery consumption, the diversity of smartphone sensing capability, the non-determinism of smartphones' platforms, and the dynamic mobility of the users carrying the smartphones. These challenges are summarized through research questions as follows. 
Q.1 Data Sampling: How to sample sensory data using smartphones in the regions of interest while not compromising the energy consumption and accuracy?

In order to answer the first research question, Q.1, we began with Hypothesis 1: if a sensing application can piggyback on another application to acquire sensory data, it saves a lot of energy consumption while maintaining the performance, especially when many applications run simultaneously on many smartphones. Inspired by the benefits of symbiotic relationships of living beings in nature, we validate this hypothesis by presenting a symbiotic sensing paradigm that is energy efficient, facilitating sharing of resources, and avoiding conflicts among sensing applications. Through theoretical and experimental analysis, the proposed symbiotic sensing showed that Hypothesis 1 is correct. Details of the answer to Q. 1 based on Hypothesis 1 were presented in Chapter 3 and summarized in Contribution 1 (Section 7.1).

To answer Q.1, we also presented an adaptive sampling method based on Hypothesis 2: sampling data only around the moment when the interesting context changes saves energy consumption while still providing sufficient informative samples. We called the adaptive sampling algorithm Minimum Active Duration Sensing Scheduling (MASS), which can effectively sample data around the change points of context that convey most information of interesting events. The key component of MASS is the Frequency Likelihood Estimation (FLE) proposed in Chapter 4. The FLE algorithm is lightweight and reliable to detect change points even if the context is dynamic and complex. Details of the adaptive sampling scheme were presented in Chapter 4 and summarized in Contribution 2 (Section 7.1).

Q.2 Data Processing: How to process the sampled data using smartphones in the regions of interest while dealing with considerable uncertainties in individual measurements?

We solved the second research question, Q. 2, with Hypotheses 3 and 4. On one hand, Hypothesis 3 says that the quality of data sampled with smartphone platforms can be enhanced by aggregating the data from multiple smartphones in the neighborhood with regard to the same event. On the other hand, Hypothesis 4 claims that the whole distribution of data sampled with smartphone platforms can be expressed by some set of model parameters. Splitting the dataset into subsets would help to weed out the low quality measurements to improve the accuracy of the system since subsets with poor measurements do not fit the model. Both hypotheses are evaluated through Chapter 5 , which present cooperative distributed data 
processing based on our non-deterministic approaches. In particular, measurement data are first combined from multiple mobile devices into a dataset. The dataset is then classified into subsets, which have distributions with smaller variances and diverse means. Through a distributed non-deterministic algorithm, the outcomes given different subsets of dataset will be filtered to obtain the best results. Through a sound localization problem, we demonstrated that using our non-deterministic approach would significantly improve the accuracy, for example, more than $200 \%$ in our localization experiment. Details of the answers to Q. 2 are presented in Chapter 5 and summarized in Contribution 3 (Section 7.1).

Q. 3 Data Dissemination: How to disseminate the sampled and processed data using mainly smartphones while facing the intermittent end-to-end connectivity?

We addressed this research question with two hypotheses. The first one is Hypothesis 5: each type of device in HSNs has its own characteristics with regard to the capability of delivering messages through the networks. Unifying different types of devices would provide better performance of message delivery than using only one type of device. To validate this hypothesis, we presented the unified routing for data dissemination that combines the advantages of different kinds of devices to efficiently deliver messages to their destinations. The simulation results given realistic settings and models demonstrated that our unified routing outperforms other well-known algorithms in terms of delivery ratio and latency.

To improve performance of message delivery, we came up with Hypothesis 6: although precisely recognizing movement patterns of mobile devices is a hard problem, combining a store-carry-forward paradigm with historical data such as the user locations and contact times would improve data dissemination performance. To test Hypothesis 6, we proposed an in-network processing routing algorithm based on machine learning techniques. Given observations on locations and contact times of smartphone users, we applied online unsupervised learning techniques to predict nodes that can deliver a message to its destination with high probability. Simulation results validated Hypothesis 6 by showing that the proposed algorithms could achieve better routing performance in terms of delivery ratio, latency, transmission cost, and computation efficiency compared to the contact-based approaches. Details of the answers to Q. 3 were presented in Chapter 6 and summarized in Contribution 3 (Section 7.1).

In conclusion, this dissertation provides key insights and contributions for meeting stringent requirements of mobile sensing in the context of HSNs. With our work, we demonstrated that smartphones are an asset to be exploited to 
meet application requirements even though they are not designed as dedicated sensor devices. In Chapter 3, we demonstrated that sharing and exchanging sensing inputs and outcomes, like symbiosis in nature, help sensing applications to avoid conflict as well as to improve the performance. We also demonstrated that such symbiotic sensing can save energy consumption while still maintaining a high probability of success in completing sensing tasks. With cooperative data sampling in Chapter 4, we showed that lightweight and energy efficient solutions to detect change points of sensing context can enable adaptive sampling schemes through sharing sensors among smartphones. With non-deterministic data processing methods presented in Chapter 5, we showed that neighboring smartphones can collaborate to provide significant improvement in accuracy when using a non-deterministic approach with a problem of sound source localization. With robust data dissemination in Chapter 6, we explored the capabilities and behaviours of each type of mobile device to disseminate sensing data and messages in HSNs. We also illustrated the effectiveness in using machine learning techniques on user movement predictions to significantly increase delivery ratios and decrease delivery latency. All of the work in this dissertation maximizes the sensing capability of smartphones to meet application or user requirements while avoiding the resource conflict among applications by using symbiotic sensing.

\subsection{Future Research Directions}

In this section, we suggest potential open research areas for both the work we have proposed as well as for smartphone-based sensing systems in the concept of the Heterogeneous Sensor Networks and Internet of Things in general.

\section{Non-deterministic Parametric Modelling}

Although the smartphone-based platforms are non-deterministic, it is interesting if the performance of smartphones can be modelled through a set of parameters given the hardware and software characteristics, for instance, the smartphone's model, the hardware configuration, and the running applications. As the first step to build these models, we have extended the framework that was used to measure audio data presented in Section 5.2 so that we can measure the latency of the audio round trip while logging most statuses of the smartphone. The statuses include the capacity of the unused memory, the frequency of the processors, and the number of applications. Once we obtain sufficient data, we 
hope to find a parametric model that can represent for the non-determinism of a specific smartphone model or brand. With the learned parameters, the accuracy of data processing will be significantly improved.

\section{Symbiotic Sensing Paradigms}

The symbiotic sensing paradigm is developed based on the symbiotic relationship of living beings in nature. However, the symbiotic sensing paradigms have not been fully exploited yet. With the rise of Internet of Things and Big Data, it is likely that sensing applications will increase significantly. To be able to be accepted by users, sensing applications have to be able to adapt themselves to the environment where the applications are installed and run. Nevertheless, current mobile operating systems such as Android does not support efficently sharing sensory data among applications. A cross-application platform as well as sharing framework are necessary to enable the deployment of symbiotic sensing applications, which can cope with security and privacy issues.

\section{Free Delay and Bandwidth for Locally Cooperative Data Processing}

Cooperative distributed processing sensory data in real time within a group of smartphones requires a local network that is able to provide high throughput. From user's perspective, extra mobile Internet fee caused by sensing processes is also a main concern, which may limits the size of participation. In this dissertation, we assume that we use a local WiFi network to exchange triggers and data for sampling and processing. In practical, such WiFi network is not alway available and does cost Internet fees. Therefore, a local network that has high throughput and low latency, but does not charge the subscription fees, is demanding. Using the network discovery service on Android platforms is of potential solution since it provides high throughput, low latency, and does not consume Internet fee.

\section{Cooperative Distributed Online Machine Learning}

In Chapter 5, we have plotted guidelines to cope with non-deterministic platforms using non-deterministic approach through the case study of the sound localization. The non-deterministic scheme also can be applied to provide inputs for a machine learning algorithm to classify an interesting event. In addition, it would be interesting to apply incremental learning algorithms to 
the context of smartphone-based sensing to cope with the dynamics. When a smartphone senses a new event that was not labelled or unclassified, it may ask the help from other smartphones if they already encountered such event. If not, the smartphone should ask the custodian with a list of suggestions based on the prediction probabilities. By doing so, the sensing system can incrementally learn the new event as well as solidify their knowledge about the old events.

\section{Machine Learning for Data Dissemination}

Although in-network routing for efficient message delivery has gained interest during last few years, using machine learning to exploit the capability of message delivery is still in its infancy. In Chapter 6 we showed that even with a simple machine learning algorithm like the k-mean clustering (KNN), the delivery ratio is better than other conventional DTN routing algorithms. Therefore, it is promising to study more advanced machine learning algorithms in the context of DTN routing.

\section{Incentives and Privacy}

One thing this dissertation and most existing work have not seriously touched is the incentives and privacy for the users who participate in smartphone-based sensing systems. To convince smartphones' users to install the sensing applications, the benefits and performances of the applications probably are not attractive enough. Incentives such as money or game credits are one of the possible solutions. In addition, preserving the privacy of users is also a key element to avoid pushing the users away from installing the sensing applications. Symbiotic sensing also raises new challenges in protecting privacy of applications and users since it requires to share data among applications. 


\section{Bibliography}

[1] A. T. Campbell, S. B. Eisenman, N. D. Lane, E. Miluzzo, and R. A. Peterson, "People-centric urban sensing," in Proceedings of the 2 nd annual international workshop on Wireless internet. ACM, 2006, p. 18.

[2] J. A. Burke, D. Estrin, M. Hansen, A. Parker, N. Ramanathan, S. Reddy, and M. B. Srivastava, "Participatory sensing," Center for Embedded Network Sensing, 2006.

[3] "So many apps, so much more time for entertainment," 2015. [Online]. Available: http://www.nielsen.com/us/en/insights/news/2015/ so-many-apps-so-much-more-time-for-entertainment.html

[4] K. Nagamine, "Idc worldwide quarterly mobile phone tracker," http:/ / www.idc.com/getdoc.jsp?containerId=prUS25860315, August 2015.

[5] N. D. Lane, S. B. Eisenman, M. Musolesi, E. Miluzzo, and A. T. Campbell, “Urban sensing systems: opportunistic or participatory?" in Proceedings of the 9th workshop on Mobile computing systems and applications. ACM, 2008, pp. 11-16.

[6] N. D. Lane, E. Miluzzo, H. Lu, D. Peebles, T. Choudhury, and A. T. Campbell, "A survey of mobile phone sensing," Communications Magazine, IEEE, vol. 48, no. 9, pp. 140-150, 2010.

[7] V.-D. Le, H. Scholten, and P. Havinga, "Online change detection for energyefficient mobile crowdsensing," in Mobile Web Information Systems, ser. Lecture Notes in Computer Science, I. Awan, M. Younas, X. Franch, and C. Quer, Eds. Springer International Publishing, 2014, vol. 8640, pp. 1-16.

[8] D. Yang, G. Xue, X. Fang, and J. Tang, "Crowdsourcing to smartphones: incentive mechanism design for mobile phone sensing," in Proceedings of the 18th annual international conference on Mobile computing and networking. ACM, 2012, pp. 173184.

[9] S. Reddy, A. Parker, J. Hyman, J. Burke, D. Estrin, and M. Hansen, “Image browsing, processing, and clustering for participatory sensing: lessons from a dietsense prototype," in Proceedings of the 4th workshop on Embedded networked sensors. ACM, 2007, pp. 13-17.

[10] J. Dai, X. Bai, Z. Yang, Z. Shen, and D. Xuan, "Perfalld: A pervasive fall detection system using mobile phones," in Pervasive Computing and Communications Workshops (PERCOM Workshops), 2010 8th IEEE International Conference on. IEEE, 2010, pp. 292-297. 
[11] N. Oliver and F. Flores-Mangas, "Healthgear: automatic sleep apnea detection and monitoring with a mobile phone," Journal of Communications, vol. 2, no. 2, pp. $1-9,2007$.

[12] K. K. Rachuri, C. Mascolo, M. Musolesi, and P. J. Rentfrow, “Sociablesense: exploring the trade-offs of adaptive sampling and computation offloading for social sensing," in Proceedings of the 17th annual international conference on Mobile computing and networking. ACM, 2011, pp. 73-84.

[13] E. Miluzzo, C. T. Cornelius, A. Ramaswamy, T. Choudhury, Z. Liu, and A. T. Campbell, "Darwin phones: the evolution of sensing and inference on mobile phones," in Proceedings of the 8th international conference on Mobile systems, applications, and services. ACM, 2010, pp. 5-20.

[14] E. Miluzzo, T. Wang, and A. T. Campbell, "Eyephone: activating mobile phones with your eyes," in Proceedings of the second ACM SIGCOMM workshop on Networking, systems, and applications on mobile handhelds. ACM, 2010, pp. 15-20.

[15] A. Sashima, Y. Inoue, T. Ikeda, T. Yamashita, and K. Kurumatani, “Consorts-s: A mobile sensing platform for context-aware services," in Intelligent Sensors, Sensor Networks and Information Processing, 2008. ISSNIP 2008. International Conference on. IEEE, 2008, pp. 417-422.

[16] K. Sha, G. Zhan, W. Shi, M. Lumley, C. Wiholm, and B. Arnetz, “Spa: a smart phone assisted chronic illness self-management system with participatory sensing," in Proceedings of the 2nd International Workshop on Systems and Networking Support for Health Care and Assisted Living Environments. ACM, 2008, p. 5.

[17] T. Denning, A. Andrew, R. Chaudhri, C. Hartung, J. Lester, G. Borriello, and G. Duncan, "Balance: towards a usable pervasive wellness application with accurate activity inference," in Proceedings of the 10th workshop on Mobile Computing Systems and Applications. ACM, 2009, p. 5.

[18] S. Consolvo, D. W. McDonald, and J. A. Landay, “Theory-driven design strategies for technologies that support behavior change in everyday life," in Proceedings of the SIGCHI Conference on Human Factors in Computing Systems. ACM, 2009, pp. 405-414.

[19] P. Jarvinen, T. H. Jarvinen, L. Lahteenmaki, and C. Sodergard, “Hyperfit: hybrid media in personal nutrition and exercise management," in Pervasive Computing Technologies for Healthcare, 2008. PervasiveHealth 2008. Second International Conference on. IEEE, 2008, pp. 222-226.

[20] C. Gao, F. Kong, and J. Tan, "Healthaware: Tackling obesity with health aware smart phone systems," in Robotics and Biomimetics (ROBIO), 2009 IEEE International Conference on. Ieee, 2009, pp. 1549-1554.

[21] C. Liao, Q. Liu, B. Liew, and L. Wilcox, "Pacer: fine-grained interactive paper via camera-touch hybrid gestures on a cell phone," in Proceedings of the SIGCHI Conference on Human Factors in Computing Systems. ACM, 2010, pp. 2441-2450. 
[22] J. J. Oresko, Z. Jin, J. Cheng, S. Huang, Y. Sun, H. Duschl, and A. C. Cheng, "A wearable smartphone-based platform for real-time cardiovascular disease detection via electrocardiogram processing," Information Technology in Biomedicine, IEEE Transactions on, vol. 14, no. 3, pp. 734-740, 2010.

[23] K. K. Rachuri, M. Musolesi, C. Mascolo, P. J. Rentfrow, C. Longworth, and A. Aucinas, "Emotionsense: a mobile phones based adaptive platform for experimental social psychology research," in Proceedings of the 12th ACM international conference on Ubiquitous computing. ACM, 2010, pp. 281-290.

[24] E. Miluzzo, N. D. Lane, K. Fodor, R. Peterson, H. Lu, M. Musolesi, S. B. Eisenman, X. Zheng, and A. T. Campbell, "Sensing meets mobile social networks: The design, implementation and evaluation of the cenceme application," in Proceedings of the 6th ACM Conference on Embedded Network Sensor Systems, ser. SenSys '08. New York, NY, USA: ACM, 2008, pp. 337-350.

[25] X. Bao and R. Roy Choudhury, "Movi: mobile phone based video highlights via collaborative sensing," in Proceedings of the 8th international conference on Mobile systems, applications, and services. ACM, 2010, pp. 357-370.

[26] T. Das, P. Mohan, V. N. Padmanabhan, R. Ramjee, and A. Sharma, "Prism: platform for remote sensing using smartphones," in Proceedings of the 8th international conference on Mobile systems, applications, and services. ACM, 2010, pp. 63-76.

[27] P. G. Kannan, S. P. Venkatagiri, M. C. Chan, A. L. Ananda, and L.-S. Peh, "Low cost crowd counting using audio tones," in Proceedings of the 10th ACM Conference on Embedded Network Sensor Systems. ACM, 2012, pp. 155-168.

[28] S. Isaacman, R. Becker, R. Cáceres, M. Martonosi, J. Rowland, A. Varshavsky, and W. Willinger, "Human mobility modeling at metropolitan scales," in Proceedings of the 10th international conference on Mobile systems, applications, and services. ACM, 2012, pp. 239-252.

[29] M. B. Kjærgaard, M. Wirz, D. Roggen, and G. Tröster, "Mobile sensing of pedestrian flocks in indoor environments using wifi signals," in Pervasive Computing and Communications (PerCom), 2012 IEEE International Conference on. IEEE, 2012, pp. 95-102.

[30] A. Beach, M. Gartrell, S. Akkala, J. Elston, J. Kelley, K. Nishimoto, B. Ray, S. Razgulin, K. Sundaresan, B. Surendar et al., "Whozthat? evolving an ecosystem for context-aware mobile social networks," Network, IEEE, vol. 22, no. 4, pp. 50-55, 2008.

[31] M. Bilandzic, M. Banholzer, D. Peev, V. Georgiev, F. Balagtas-Fernandez, and A. De Luca, "Laermometer: a mobile noise mapping application," in Proceedings of the 5th Nordic conference on Human-computer interaction: building bridges. ACM, 2008, pp. 415-418. 
[32] M. Mun, S. Reddy, K. Shilton, N. Yau, J. Burke, D. Estrin, M. Hansen, E. Howard, R. West, and P. Boda, "Peir, the personal environmental impact report, as a platform for participatory sensing systems research," in Proceedings of the 7th international conference on Mobile systems, applications, and services. ACM, 2009, pp. $55-68$.

[33] R. K. Rana, C. T. Chou, S. S. Kanhere, N. Bulusu, and W. Hu, “Ear-phone: an end-to-end participatory urban noise mapping system," in Proceedings of the 9th ACM/IEEE International Conference on Information Processing in Sensor Networks. ACM, 2010, pp. 105-116.

[34] S. Gaonkar, J. Li, R. R. Choudhury, L. Cox, and A. Schmidt, "Micro-blog: sharing and querying content through mobile phones and social participation," in Proceedings of the 6th international conference on Mobile systems, applications, and services. ACM, 2008, pp. 174-186.

[35] H. Lu, W. Pan, N. D. Lane, T. Choudhury, and A. T. Campbell, "Soundsense: scalable sound sensing for people-centric applications on mobile phones," in Proceedings of the 7th international conference on Mobile systems, applications, and services. ACM, 2009, pp. 165-178.

[36] E. Kanjo, S. Benford, M. Paxton, A. Chamberlain, D. S. Fraser, D. Woodgate, D. Crellin, and A. Woolard, "Mobgeosen: facilitating personal geosensor data collection and visualization using mobile phones," Personal and Ubiquitous Computing, vol. 12, no. 8, pp. 599-607, 2008.

[37] N. Maisonneuve, M. Stevens, M. E. Niessen, and L. Steels, “Noisetube: Measuring and mapping noise pollution with mobile phones," in Information Technologies in Environmental Engineering. Springer, 2009, pp. 215-228.

[38] Y. Chon, E. Talipov, H. Shin, and H. Cha, "Smartdc: Mobility prediction-based adaptive duty cycling for everyday location monitoring," Mobile Computing, IEEE Transactions on, vol. 13, no. 3, pp. 512-525, 2014.

[39] N. D. Lane, P. Georgiev, and L. Qendro, "Deepear: robust smartphone audio sensing in unconstrained acoustic environments using deep learning," in Proceedings of the 2015 ACM International Joint Conference on Pervasive and Ubiquitous Computing. ACM, 2015, pp. 283-294.

[40] W. Willett, P. Aoki, N. Kumar, S. Subramanian, and A. Woodruff, “Common sense community: scaffolding mobile sensing and analysis for novice users," in Pervasive Computing. Springer, 2010, pp. 301-318.

[41] Y. Jiang, K. Li, L. Tian, R. Piedrahita, X. Yun, O. Mansata, Q. Lv, R. P. Dick, M. Hannigan, and L. Shang, "Maqs: a personalized mobile sensing system for indoor air quality monitoring," in Proceedings of the 13th international conference on Ubiquitous computing. ACM, 2011, pp. 271-280. 
[42] R. W. Ouyang, A. Srivastava, P. Prabahar, R. Roy Choudhury, M. Addicott, and F. J. McClernon, "If you see something, swipe towards it: crowdsourced event localization using smartphones," in Proceedings of the 2013 ACM international joint conference on Pervasive and ubiquitous computing. ACM, 2013, pp. 23-32.

[43] H. Wang, S. Sen, A. Elgohary, M. Farid, M. Youssef, and R. R. Choudhury, "No need to war-drive: unsupervised indoor localization," in Proceedings of the 10th international conference on Mobile systems, applications, and services. ACM, 2012, pp. 197-210.

[44] B. Hull, V. Bychkovsky, Y. Zhang, K. Chen, M. Goraczko, A. Miu, E. Shih, H. Balakrishnan, and S. Madden, "Cartel: a distributed mobile sensor computing system," in Proceedings of the 4th international conference on Embedded networked sensor systems. ACM, 2006, pp. 125-138.

[45] F. Zhang, D. Wilkie, Y. Zheng, and X. Xie, "Sensing the pulse of urban refueling behavior," in Proceedings of the 2013 ACM international joint conference on Pervasive and ubiquitous computing. ACM, 2013, pp. 13-22.

[46] R. K. Ganti, N. Pham, H. Ahmadi, S. Nangia, and T. F. Abdelzaher, “Greengps: a participatory sensing fuel-efficient maps application," in Proceedings of the 8th international conference on Mobile systems, applications, and services. ACM, 2010, pp. 151-164.

[47] S. Mathur, T. Jin, N. Kasturirangan, J. Chandrasekaran, W. Xue, M. Gruteser, and W. Trappe, "Parknet: drive-by sensing of road-side parking statistics," in Proceedings of the 8th international conference on Mobile systems, applications, and services. ACM, 2010, pp. 123-136.

[48] A. Janecek, K. A. Hummel, D. Valerio, F. Ricciato, and H. Hlavacs, “Cellular data meet vehicular traffic theory: location area updates and cell transitions for travel time estimation," in Proceedings of the 2012 ACM Conference on Ubiquitous Computing. ACM, 2012, pp. 361-370.

[49] P. Zhou, Y. Zheng, and M. Li, "How long to wait?: predicting bus arrival time with mobile phone based participatory sensing," in Proceedings of the 10th international conference on Mobile systems, applications, and services. ACM, 2012, pp. 379-392.

[50] Y. Maekawa, A. Uchiyama, H. Yamaguchi, and T. Higashino, "Car-level congestion and position estimation for railway trips using mobile phones," in Proceedings of the 2014 ACM International Joint Conference on Pervasive and Ubiquitous Computing. ACM, 2014, pp. 939-950.

[51] J. Weppner and P. Lukowicz, "Bluetooth based collaborative crowd density estimation with mobile phones," in Pervasive computing and communications (PerCom), 2013 IEEE international conference on. IEEE, 2013, pp. 193-200.

[52] T. Nishimura, T. Higuchi, H. Yamaguchi, and T. Higashino, "Detecting smoothness of pedestrian flows by participatory sensing with mobile phones," in Pro- 
ceedings of the 2014 ACM International Symposium on Wearable Computers. ACM, 2014, pp. 15-18.

[53] A. Thiagarajan, L. Ravindranath, K. LaCurts, S. Madden, H. Balakrishnan, S. Toledo, and J. Eriksson, "Vtrack: accurate, energy-aware road traffic delay estimation using mobile phones," in Proceedings of the 7th ACM Conference on Embedded Networked Sensor Systems. ACM, 2009, pp. 85-98.

[54] P. Mohan, V. N. Padmanabhan, and R. Ramjee, "Nericell: using mobile smartphones for rich monitoring of road and traffic conditions," in Proceedings of the 6th ACM conference on Embedded network sensor systems. ACM, 2008, pp. 357-358.

[55] C. Cornelius, A. Kapadia, D. Kotz, D. Peebles, M. Shin, and N. Triandopoulos, "Anonysense: privacy-aware people-centric sensing," in Proceedings of the 6th international conference on Mobile systems, applications, and services. ACM, 2008, pp. 211-224.

[56] H. Lu, N. D. Lane, S. B. Eisenman, and A. T. Campbell, "Bubble-sensing: Binding sensing tasks to the physical world," Pervasive and Mobile Computing, vol. 6, no. 1, pp. 58-71, 2010.

[57] "Number of available applications in the google play store from december 2009 to november 2015," 2015. [Online]. Available: http://www.statista.com/ statistics/266210/number-of-available-applications-in-the-google-play-store/

[58] "Number of available apps in the apple app store from july 2008 to june 2015," 2015. [Online]. Available: http://www.statista.com/statistics/263795/ number-of-available-apps-in-the-apple-app-store/

[59] T. M. I. Team, "Average britons spends almost 34 entire days on mobile phone per year," http:/ / www.mobileinsurance.co.uk/blog/average-britonsspends-almost-34-entire-days-on-mobile-phone-per-year/, March 2013.

[60] J. M. Brick, P. D. Brick, S. Dipko, S. Presser, C. Tucker, and Y. Yuan, “Cell phone survey feasibility in the us: Sampling and calling cell numbers versus landline numbers," Public Opinion Quarterly, vol. 71, no. 1, pp. 23-39, 2007.

[61] K. K. Rachuri, M. Musolesi, C. Mascolo, P. J. Rentfrow, C. Longworth, and A. Aucinas, "Emotionsense: A mobile phones based adaptive platform for experimental social psychology research," in Proceedings of the 12th ACM International Conference on Ubiquitous Computing, ser. Ubicomp '10. New York, NY, USA: ACM, 2010, pp. 281-290.

[62] W. Sherchan, P. P. Jayaraman, S. Krishnaswamy, A. Zaslavsky, S. Loke, and A. Sinha, "Using on-the-move mining for mobile crowdsensing," in Proceedings of the 2012 IEEE 13th International Conference on Mobile Data Management (Mdm 2012), ser. MDM '12. Washington, DC, USA: IEEE Computer Society, 2012, pp. $115-124$. 
[63] A. Beach, M. Gartrell, S. Akkala, J. Elston, J. Kelley, K. Nishimoto, B. Ray, S. Razgulin, K. Sundaresan, B. Surendar, M. Terada, and R. Han, "Whozthat? evolving an ecosystem for context-aware mobile social networks," Netwrk. Mag. of Global Internetwkg., vol. 22, no. 4, pp. 50-55, Jul. 2008.

[64] Y. Wang, J. Lin, M. Annavaram, Q. A. Jacobson, J. Hong, B. Krishnamachari, and N. Sadeh, "A framework of energy efficient mobile sensing for automatic user state recognition," in Proceedings of the 7th International Conference on Mobile Systems, Applications, and Services, ser. MobiSys '09. New York, NY, USA: ACM, 2009, pp. 179-192.

[65] H. Lu, J. Yang, Z. Liu, N. D. Lane, T. Choudhury, and A. T. Campbell, “The jigsaw continuous sensing engine for mobile phone applications," in Proceedings of the 8th ACM Conference on Embedded Networked Sensor Systems, ser. SenSys '10. New York, NY, USA: ACM, 2010, pp. 71-84.

[66] V. Chandola, A. Banerjee, and V. Kumar, "Anomaly detection: A survey," $A C M$ Comput. Surv., vol. 41, no. 3, pp. 15:1-15:58, 2009.

[67] _ - "Anomaly detection for discrete sequences: A survey," Knowledge and Data Engineering, IEEE Transactions on, vol. 24, no. 5, pp. 823-839, 2012.

[68] L. M. Manevitz and M. Yousef, "One-class svms for document classification," J. Mach. Learn. Res., vol. 2, pp. 139-154, 2002.

[69] A. Ng, "Machine learning," https://class.coursera.org/ml/lecture/97.

[70] M. Goldstein and A. Dengel., "Histogram-based outlier score (hbos): A fast unsupervised anomaly detection algorithm," in In Stefan Advances in Konwledge Discovery and Data Mining, ser. Lecture Notes in Computer Science. Springer, 206, pp. 577-593.

[71] V. D. Le, H. Scholten, and P. Havinga, “Flead: Online frequency likelihood estimation anomaly detection for mobile sensing," in Proceedings of the 2013 ACM Conference on Pervasive and Ubiquitous Computing Adjunct Publication, ser. UbiComp '13 Adjunct. New York, NY, USA: ACM, 2013, pp. 1159-1166.

[72] D. L. Olson and D. Delen, Advanced DataMining Techniques. Springer, 2008.

[73] T. Giannakopoulos and A. Pikrakis, Introduction to Audio Analysis: A MATLAB® Approach. Academic Press, 2014.

[74] V. D. Le, H. Scholten, and P. Havinga, "Flead: Online frequency likelihood estimation anomaly detection for mobile sensing," in Proceedings of the 2013 ACM conference on Pervasive and ubiquitous computing adjunct publication. ACM, 2013, pp. 1159-1166.

[75] "Findsounds," 2015. [Online]. Available: http://www.findsounds.com

[76] L. Perneel, H. Fayyad-Kazan, and M. Timmerman, "Can android be used for realtime purposes?" in Computer Systems and Industrial Informatics (ICCSII), 2012 International Conference on. IEEE, 2012, pp. 1-6. 
[77] "Audio latency measurements." [Online]. Available: https://source.android. com/devices/audio/latency_measurements.html

[78] J. J. Moré, "The levenberg-marquardt algorithm: implementation and theory," in Numerical analysis. Springer, 1978, pp. 105-116.

[79] P. Bloomfield and W. Steiger, "Least absolute deviations, theory, applications," Algorithms, 1983.

[80] M. A. Fischler and R. C. Bolles, "Random sample consensus: a paradigm for model fitting with applications to image analysis and automated cartography," Communications of the ACM, vol. 24, no. 6, pp. 381-395, 1981.

[81] I. Kalkov, A. Gurghian, and S. Kowalewski, "Predictable broadcasting of parallel intents in real-time android," in Proceedings of the 12th International Workshop on Java Technologies for Real-time and Embedded Systems. ACM, 2014, p. 57.

[82] Y. Yan, S. Cosgrove, V. Anand, A. Kulkarni, S. H. Konduri, S. Y. Ko, and L. Ziarek, "Real-time android with rtdroid," in Proceedings of the 12th annual international conference on Mobile systems, applications, and services. ACM, 2014, pp. 273-286.

[83] A. Armoush, D. Franke, I. Kalkov, and S. Kowalewski, "An approach for using mobile devices in industrial safety-critical embedded systems," Mobile Computing, Applications, and Services, pp. 294-297, 2014.

[84] K. Liu, X. Liu, L. Xie, and X. Li, "Towards accurate acoustic localization on a smartphone," in INFOCOM, 2013 Proceedings IEEE, April 2013, pp. 495-499.

[85] T. F. Coleman and Y. Li, "An interior trust region approach for nonlinear minimization subject to bounds," SIAM Journal on optimization, vol. 6, no. 2, pp. 418$445,1996$.

[86] V.-D. Le, H. Scholten, and P. M. Havinga, “Online change detection for energyefficient mobile crowdsensing," in Mobile Web Information Systems. Springer, 2014, pp. 1-16.

[87] N. Patwari, J. N. Ash, S. Kyperountas, A. O. Hero III, R. L. Moses, and N. S. Correal, "Locating the nodes: cooperative localization in wireless sensor networks," Signal Processing Magazine, IEEE, vol. 22, no. 4, pp. 54-69, 2005.

[88] D. Camara, C. Bonnet, and F. Filali, "Propagation of public safety warning message: A delay tolerant approach," in Proc. IEEE Communications Society WCNC, 2010.

[89] A. T. Erman, A. Dilo, and P. Havinga, "A fault-tolerant data dissemination based on honeycomb architecture for mobile multi-sink wireless sensor networks," in Proc. of the Sixth International Conference on Intelligent Sensors, Sensor Networks and Information Processing, ISSNIP 2010, 2010, pp. 97-102.

[90] T. Spyropoulos, K. Psounis, and C. S. Raghavendra, "Efficient routing in intermittently connected mobile networks: the multiple-copy case," IEEE/ACM Trans. Netw., vol. 16, no. 1, pp. 77-90, Feb. 2008. 
[91] R. Schwartz, R. Barbosa, N. Meratnia, G. Heijenk, and J. Scholten, "A directional data dissemination protocol for vehicular environments," Computer Communications, vol. 34, pp. 2057-2071, 2011.

[92] B. K. Polat, P. Sachdeva, M. H. Ammar, and E. W. Zegura, "Message ferries as generalized dominating sets in intermittently connected mobile networks," Pervasive and Mobile Computing, vol. 7, pp. 189-205, 2011.

[93] D. Borsetti, C. Casetti, C.-F. Chiasserini, M. Fiore, and J. M. Barceló-Ordinas, “Virtual data mules for data collection in road-side sensor networks," in Proceedings of the Second International Workshop on Mobile Opportunistic Networking, ser. MobiOpp '10. New York, NY, USA: ACM, 2010, pp. 32-40.

[94] A. Vahdat and D. Becker, "Epidemic routing for partially connected ad hoc networks," Department of Computer Science, Duke Univeristy, Durham, NC, Tech. Rep., 2000.

[95] T. Spyropoulos, K. Psounis, and C. Raghavendra, "Single-copy routing in intermittently connected mobile networks," in Proc. of Sensor and Ad Hoc Communications and Networks (SECON), 2004, pp. 235-244.

[96] — - "Spray and wait: An efficient routing scheme for intermittently connected mobile networks," in Proc. of ACM SIGCOMM Workshop on Delay-Tolerant Networking (WDTN), 2005.

[97] S. Jain, K. Fall, and R. Patra, "Routing in a delay tolerant network," in Proc. of ACM SIGCOMM on Wireless and Delay-Tolerant Networks, 2004.

[98] A. Lindgren and A. Droia, "Probabilistic routing protocol for intermittently connected networks," Internet Draft draft-lindgren-dtnrg-prophet-02, Work in Progress, 2006.

[99] E. M. Daly and M. Haahr, "Social network analysis for routing in disconnected delay-tolerant manets," in Proceedings of the 8th ACM international symposium on Mobile ad hoc networking and computing, ser. MobiHoc '07. New York, NY, USA: ACM, 2007, pp. 32-40.

[100] P. Hui, J. Crowcroft, and E. Yoneki, “Bubble rap: social-based forwarding in delay tolerant networks," in Proceedings of the 9th ACM international symposium on Mobile ad hoc networking and computing, ser. MobiHoc '08. New York, NY, USA: ACM, 2008, pp. 241-250.

[101] J. A. Bitsch Link, N. Viol, A. Goliath, and K. Wehrle, “Simbetage: utilizing temporal changes in social networks for pocket switched networks," in Proceedings of the 1st ACM workshop on User-provided networking: challenges and opportunities, ser. U-NET '09. New York, NY, USA: ACM, 2009, pp. 13-18.

[102] V.-D. Le, H. Scholten, and P. Havinga, "Evaluation of opportunistic routing algorithms on opportunistic mobile sensor networks with infrastructure assistance," International Journal On Advances in Networks and Services, vol. 5, no. 3 and 4, pp. 279-290, 2012. 
[103] — - "Towards opportunistic data dissemination in mobile phone sensor networks," in Proc. of The Eleventh International Conference on Networks (ICN 2012), 2012.

[104] A. Keranen, J. Ott, and T. Karkkainen, "The one simulator for dtn protocol evaluation," in Proc. of the 2nd International Conference on Simulation Tools and Techniques(SIMUTools), 2009.

[105] C. Perkins, E. Belding-Royer, and S. Das, "Ad hoc on-demand distance vector (aodv) routing," RFC Editor, United States, Tech. Rep., 2003.

[106] D. B. Johnson, D. A. Maltz, and J. Broch, "Ad hoc networking." Boston, MA, USA: Addison-Wesley Longman Publishing Co., Inc., 2001, ch. DSR: the dynamic source routing protocol for multihop wireless ad hoc networks, pp. 139-172.

[107] Y. Wang and H. Wu, "Delay/fault-tolerant mobile sensor network (dft-msn): A new paradigm for pervasive information gathering," IEEE Trans. Mobile Computing, vol. 6, pp. 1021 - 1034, 2007.

[108] Newcom++, "State of the art of research on opportunistic networks, and definition of a common framework for reference models and performance metrics," Downloaded from http://www.newcom-project.eu/publicdeliverables/research/DR11.1-final-1.pdf/view.

[109] R. Shah, S. Roy, S. Jain, and W. Brunette, “Data mules: modeling a three-tier architecture for sparse sensor networks," in Sensor Network Protocols and Applications, 2003. Proceedings of the First IEEE. 2003 IEEE International Workshop on, may 2003, pp. $30-41$.

[110] W. Zhao, M. Ammar, and E. Zegura, "A message ferrying approach for data delivery in sparse mobile ad hoc networks," in Proceedings of the 5th ACM international symposium on Mobile ad hoc networking and computing, ser. MobiHoc '04. New York, NY, USA: ACM, 2004, pp. 187-198.

[111] Y. Xian, C. Huang, and J. Cobb, "Look-ahead routing and message scheduling in delay-tolerant networks," in Proc. IEEE Conference on Local Computer Networks (LCN), 2010.

[112] A. Lindgren, T. Karkkainen, and J. Ott, "Simulating mobility and dtns with the one," Journal of Communications, 2010.

[113] A. Chaintreau, P. Hui, J. Crowcroft, C. Diot, R. Gass, and J. Scott, "Impact of human mobility on the design of opportunistic forwarding algorithms," in Proc. IEEE Infocom, 2006.

[114] F. Ekman, A. Keranen, J. Karvo, and J. Ott, "Working day movement model," in Proc. of The 1st ACM SIGMOBILE workshop on Mobility models (MobilityModels), 2008. 
[115] J. Scott, R. Gass, J. Crowcroft, P. Hui, C. Diot, and A. Chaintreau, “CRAWDAD trace cambridge/haggle/imote/infocom2006 (v. 2009-05-29)," Downloaded from http://crawdad.cs.dartmouth.edu, May 2009.

[116] J. Eriksson, H. Balakrishnan, and S. Madden, "Cabernet: Vehicular Content Delivery Using WiFi," in 14th ACM MOBICOM, San Francisco, CA, September 2008.

[117] V. Mitlin, "Optimal mac packet size in networks without cut-through routing," IEEE Trans. on Wireless Communications, vol. 2, pp. 901 - 910, 2003.

[118] J. Burgess, B. Gallagher, D. Jensen, and B. Levine, “Maxprop: Routing for vehiclebased disruption-tolerant networks," in Proc. of IEEE INFOCOM, 2006.

[119] Y. Li, M. Qian, D. Jin, L. Su, and L. Zeng, "Adaptive optimal buffer management policies for realistic dtn," in Proc. of the 28th IEEE conference on Global telecommunications (GLOBECOM'09), 2009, pp. 2683-2687.

[120] G.Fathima and R. Wahidabaru, "Buffer management for preferential delivery in opportunistic delay tolerant networks," International Journal of Wireless and Mobile Networks (IJWMN), vol. 3, pp. 15-28, 2011.

[121] R. O. Duda, P. E. Hart, and D. G. Stork, Pattern Classification. Wiley-Interscience, 2000.

[122] I. Rhee, M. Shin, S. Hong, K. Lee, S. J. Kim, and S. Chong, “On the levy-walk nature of human mobility," IEEE/ACM TRANSACTIONS ON NETWORKING, vol. 19, pp. 189-205, 2011.

[123] D. Aldous and J. Fill, Reversible markov chains and random walks on graphs. (monograph in preparation). [Online]. Available: http://www.stat.berkeley.edu/ aldous/RWG/book.html 



\section{About the author}

Lê Viết Đức joined PS as a Ph.D. candidate in June 2011. He received a Bachelor degree in Electrical-Electronics Engineering from Ho Chi Minh City University of Technology, Vietnam in 2002, and a Master degree in Computer Engineering from KyungHee University, South Korea in 2009. He is interested in exploiting Data Analysis and Pattern Recognition tools for Wireless Sensor Networks, Internet of Things, and Biologyinspired Systems that can be applied to Health-care applications, Location Tracking, Public Safety, and Smart Transportation Systems. Besides his academic research, he has more than seven years working on many industrial projects related to sensor applications and web database as a researcher or a team leader.

List of publications in which he participated in reversed chronological order.

1. D.V. Le, J.W. Kamminga, H. Scholten, and P.J.M. Havinga “Error Bounds of Localization with Noise Diversity," in Distributed Computing in Sensor Systems, 2016 IEEE International Conference on. IEEE, 2016.

2. D.V. Le, J.W. Kamminga, H. Scholten, and P.J.M. Havinga "Nondeterministic Sound Source Localization with Smartphones in Crowdsensing," in Pervasive Computing and Communications Workshops (PERCOM Workshops), 2016 IEEE International Conference on. IEEE, 2016.

3. V.-D. Le, H. Scholten, and P. M. Havinga, "Online change detection for energyefficient mobile crowdsensing," in Mobile Web Information Systems. Springer, 2014, pp. 1-16. (Best Paper Award)

4. V. D. Le, H. Scholten, P. Havinga, and H. Ngo, "Location-based data dissemination with human mobility using online density estimation," in Consumer Communications and Networking Conference (CCNC), 2014 IEEE 11th. IEEE, 2014, pp. $450-457$.

5. V. D. Le, H. Scholten, and P. Havinga, “Flead: Online frequency likelihood estimation anomaly detection for mobile sensing," in Proceedings of the 2013 ACM Conference on Pervasive and Ubiquitous Computing Adjunct Publication, ser. UbiComp '13 Adjunct. New York, NY, USA: ACM, 2013, pp. 1159-1166.

6. V.-D. Le, "Distributed opportunistic sensing in mobile phone sensor networks," in Pervasive Computing and Communications Workshops (PERCOM Workshops), 2013 IEEE International Conference on. IEEE, 2013, pp. 427-428. 
7. V.-D. Le, H. Scholten, and P. Havinga, "Evaluation of opportunistic routing algorithms on opportunistic mobile sensor networks with infrastructure assistance," International Journal On Advances in Networks and Services, vol. 5, no. 3 and 4, pp. 279-290, 2012.

8. V.-D. Le, H. Scholten, and P. Havinga, "Unified routing for data dissemination in smart city networks," in Proc. of the 3rd International Conference on the Internet of Things (IoT2012), 2012.

9. V.-D. Le Data Dissemination in Mobile Phone Sensor Networks. Technical Report TR-CTIT-14-03, Centre for Telematics and Information Technology, University of Twente, Enschede. ISSN 1381-3625, 2012

10. V.-D. Le, H. Scholten, and P. Havinga, "Towards opportunistic data dissemination in mobile phone sensor networks," in Proc. of The Eleventh International Conference on Networks (ICN 2012), 2012. (Best Paper Award)

11. V.-H. Dang, V.-D. Le, Y.-K. Lee, and S. Lee, “Distributed push-pull estimation for node localization in wireless sensor networks," Journal of Parallel and Distributed Computing, vol. 71, no. 3, pp. 471-484, 2012.

12. T.-D. Pham, H.Q. Ngo, V. D. Le, S. Lee, and Y.-K. Lee, "Broadcast Gossip Based Distributed Hypothesis Testing in Wireless Sensor Networks," in The IEEE International Conference on Advanced Technologies for Communications 2009 (ATC09), Hai Phong, Vietnam, IEEE, 2009, pp. 84-87.

13. V.-D. Le, Y.-K. Lee, and S. Lee "Localization in Sensor Networks with Fading Channels based on Nonmetric Distrance Models," in International Conference on Computational Science and its Applications (ICCSA 2009), 29 June - 02 July 2009, South Korea. pp. 419-431. Lecture Notes in Computer Science 5593. Springer Verlag. ISSN 0302-9743 ISBN 978-3-642-02456-6

14. V.-D. Le, Y.-K. Lee, S. Lee, and S.-H. Lee "Distributed Localization in Wireless Sensor Networks based on Force-Vectors," in International conference on Intelligent Sensors, Sensor Networks and Information Processing, ISSNIP 2008, 15-18 Dec 2008, Sydney, Australia. pp. 31-36. IEEE Computer Society. ISBN 978-1-4244-3822-8 


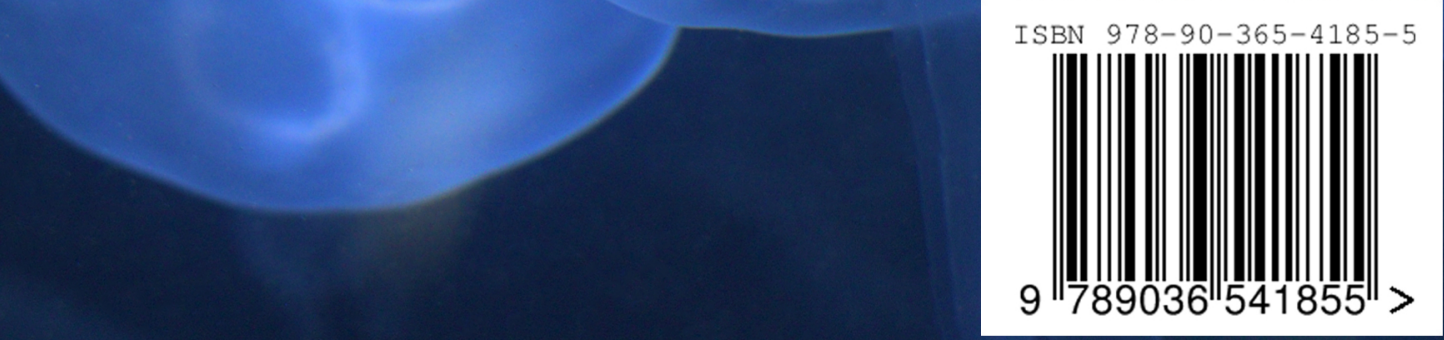